Aus der Klinik für Unfallchirurgie, Orthopädie und Plastische Chirurgie

(Prof. Dr. med. W. Lehmann)

der Medizinischen Fakultät der Universität Göttingen

\title{
Die Behandlung der kindlichen Skoliose bei spinaler Muskelatrophie mit extern zu kontrollierenden magnetischen Implantaten
}

\author{
INAUGURAL-DISSERTATION \\ zur Erlangung des Doktorgrades \\ der Medizinischen Fakultät der \\ Georg-August-Universität zu Göttingen
}

vorgelegt von

Batoul Badwan

aus

Kuwait

Göttingen 2017 
Dekan:

Referentin:

Ko-Referent:

Drittreferent:
Prof. Dr. rer. nat. H. K. Kroemer

Prof. Dr. med. A.-K. Hell

Prof. Dr. med. H.-C. Ludwig

Prof. Dr. med. dent. R. Mausberg

Datum der mündlichen Prüfung: 27.08.2018 
Hiermit erkläre ich, die Dissertation mit dem Titel "Die Behandlung der kindlichen Skoliose bei spinaler Muskelatrophie mit extern zu kontrollierenden magnetischen Implantaten" eigenständig angefertigt und keine anderen als die von mir angegebenen Quellen und Hilfsmittel verwendet zu haben.

Göttingen, den

(Unterschrift) 
Teile dieser Dissertation wurden bereits veröffentlicht:

\section{Publikation:}

Lorenz H, Badwan B, Hecker M, Tsaknakis K, Groenefeld K, Braunschweig L, Hell AK (2017): Magnetically controlled devices parallel to the spine in children with spinal muscular atrophy. JBJS Open Access $\underline{2}$, e0036 


\section{Inhaltsverzeichnis}

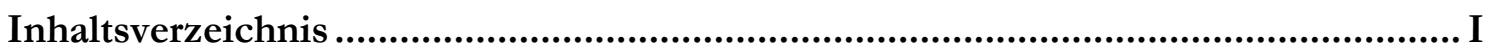

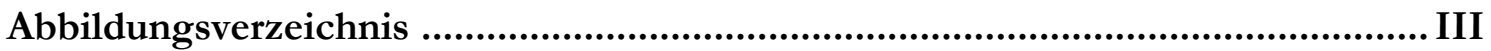

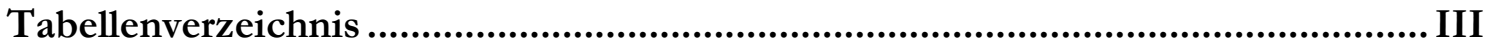

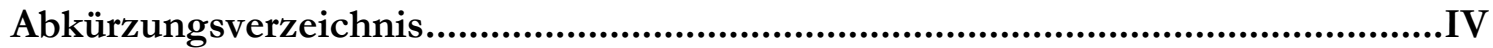

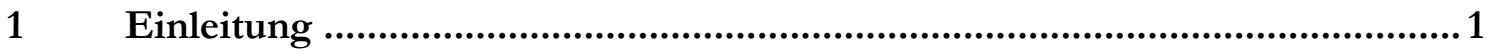

1.1 Gängige Klassifikationen der Skoliose ....................................................................................

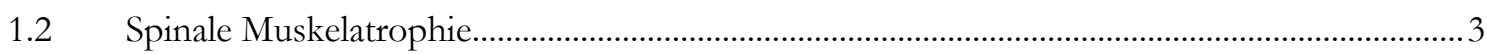

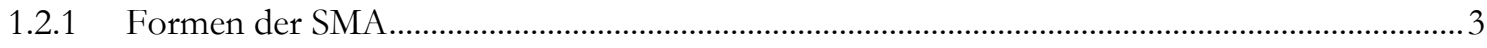

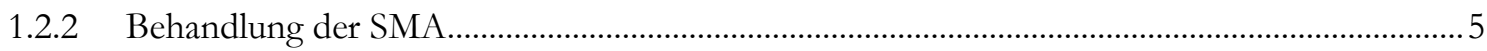

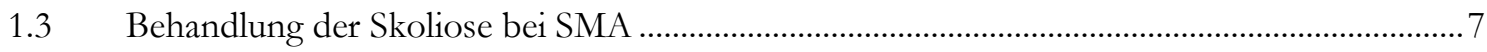

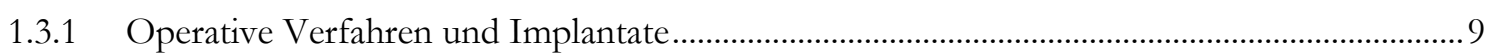

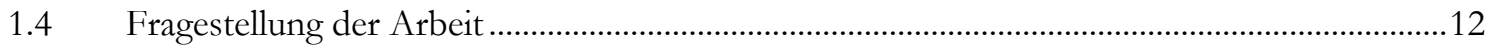

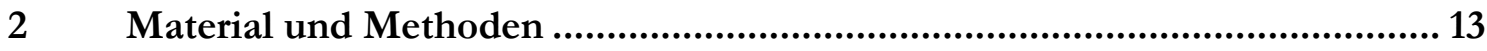

$2.1 \quad$ Ethikkommission ..............................................................................................................13

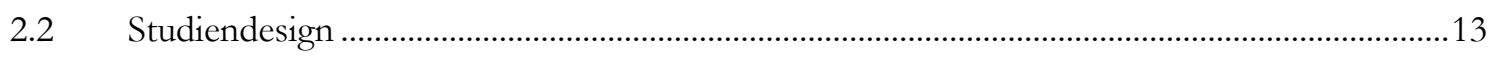

2.2.1 Ein- und Ausschlusskriterien des Studienkollektivs ................................................................13

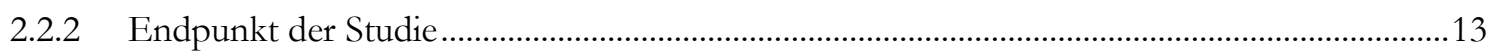

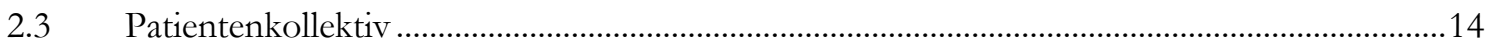

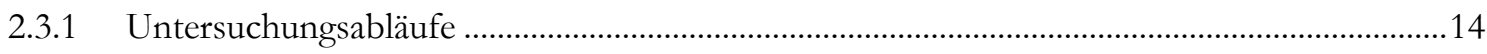

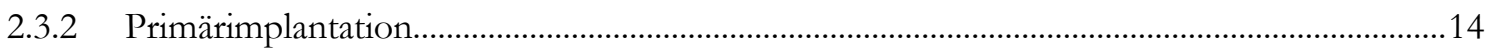

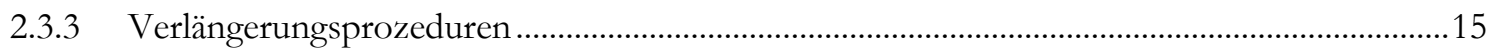

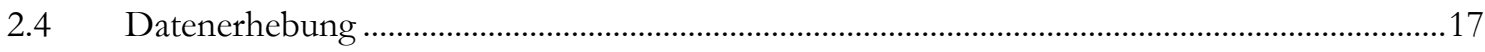

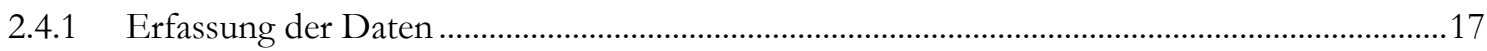

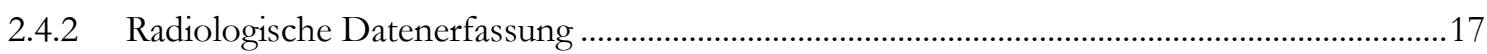

2.4.2.1 Skoliosemessung anhand des Skoliosekrümmungswinkels (Cobb-Winkel).............................18

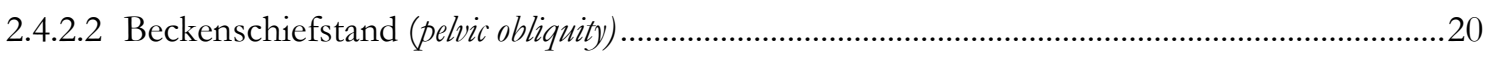

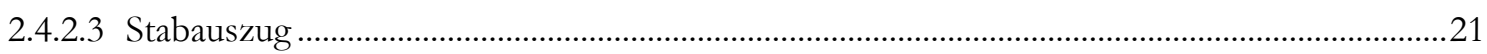

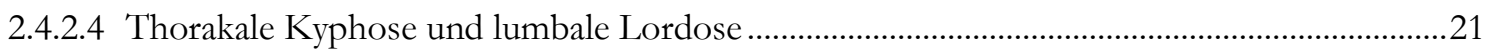

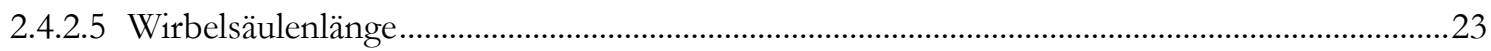

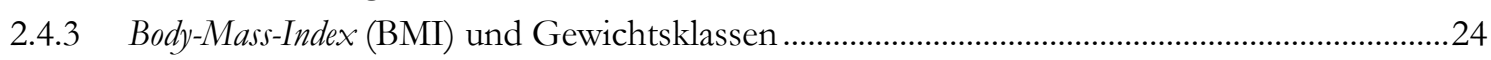

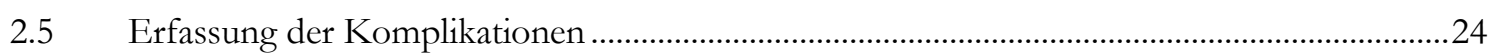

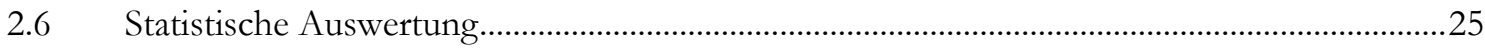

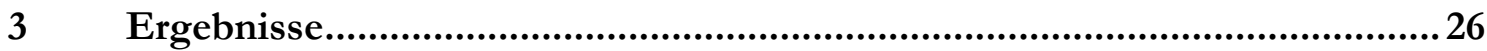

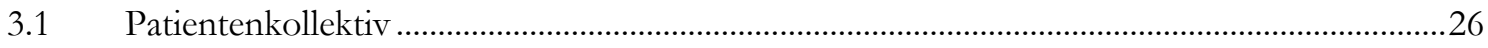

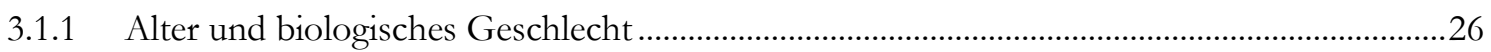




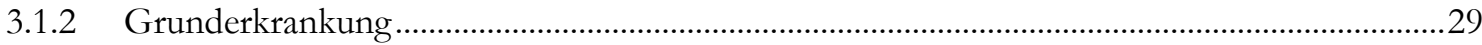

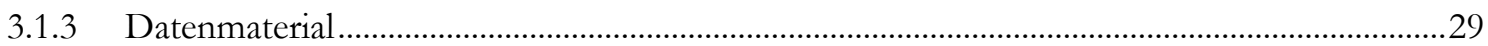

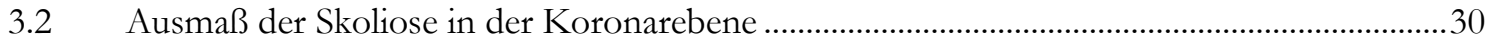

3.2.1 Skoliosekrümmungswinkel (Cobb-Winkel) des Gesamtkollektivs im Verlauf........................30

3.2.2 Skoliosekrümmungswinkel in Abhängigkeit vom Alter.......................................................32

3.2.3 Skoliosekrümmungswinkel in Abhängigkeit vom Kinder-BMI............................................33

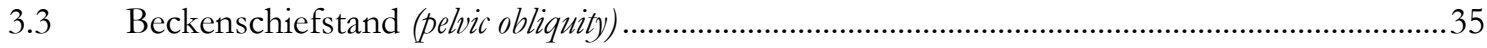

3.3.1 Beckenschiefstand des Gesamtkollektivs im Verlauf .............................................................35

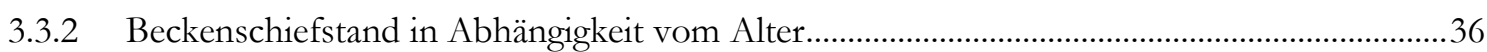

3.3.3 Beckenschiefstand in Abhängigkeit vom Kinder-BMI .......................................................37

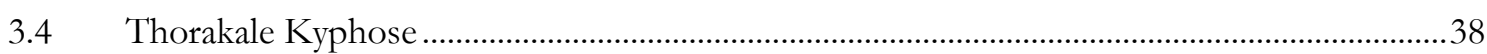

3.4.1 Thorakale Kyphose des Gesamtkollektivs im Verlauf ............................................................38

3.4.2 Thorakale Kyphose in Abhängigkeit vom Alter....................................................................4 40

3.4.3 Thorakale Kyphose in Abhängigkeit vom Kinder-BMI ........................................................41

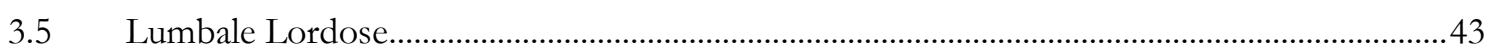

3.5.1 Lumbale Lordose des Gesamtkollektivs im Verlauf.................................................................43

3.5.2 Lumbale Lordose in Abhängigkeit vom Alter ......................................................................44

3.5.3 Lumbale Lordose in Abhängigkeit vom Kinder-BMI............................................................45

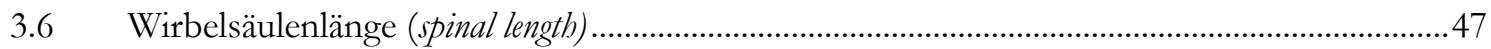

3.6.1 Wirbelsäulenlänge des Gesamtkollektivs im Verlauf................................................................47

3.6.2 Wirbelsäulenlänge in Abhängigkeit vom Alter ......................................................................49

3.6.3 Wirbelsäulenlänge in Abhängigkeit vom Kinder-BMI .............................................................50

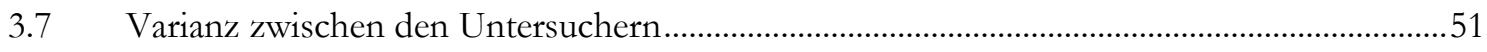

3.7.1 Varianz des Skoliosekrümmungswinkels (Cobb-Winkel) .........................................................52

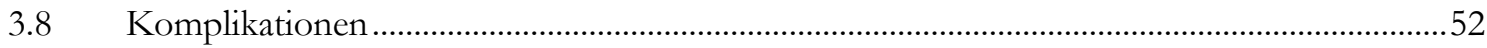

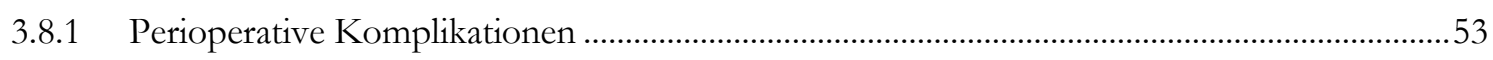

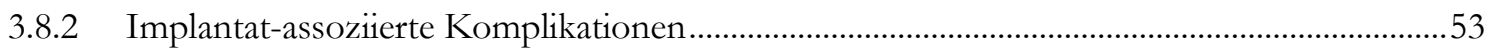

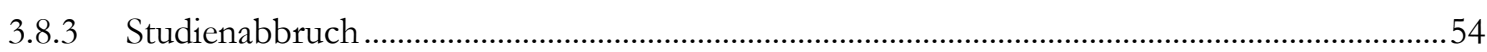

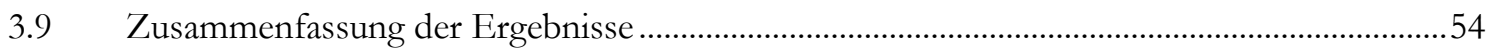

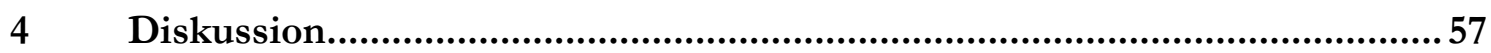

4.1 Korrektur der Wirbelsäulendeformität und des Beckenschiefstandes...................................58

4.2 Sagittales Profil- Kyphose, Lordose und Wirbelsäulenlänge...................................................60

4.3 Korrektur der Wirbelsäulendeformität im Verlauf ................................................................62

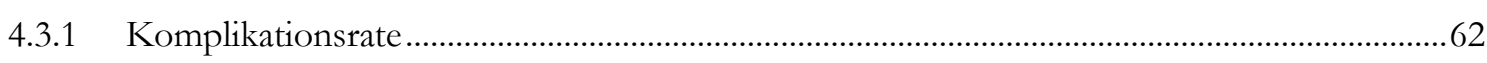

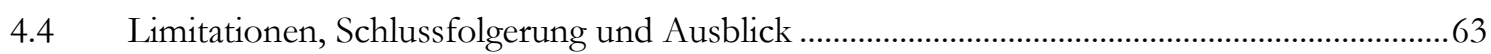

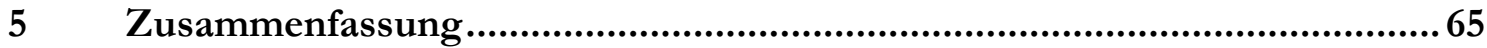

6 Literaturverzeichnis ........................................................................67 


\section{Abbildungsverzeichnis}

Abbildung 1: Röntgenaufnahmen eines 13-jährigen Kindes mit konservativ behandelter Skoliose bei SMA Typ II

Abbildung 2: Skelett mit Implantation eines MAGEC $\AA_{-}$-Stabs von dorsal..............................15

Abbildung 3: MAGEC® $W$ and Magnet Locator der Firma Ellipse Technologies......................16

Abbildung 4: MAGEC ${ }^{\circledR}$ External Remote Controller der Firma Ellipse Technologies .............17

Abbildung 5: Strahlendurchlässiger Stuhl zur Aufnahme von Röntgenbildern .......................18

Abbildung 6: Schemazeichnung zur Messung des Skoliosekrümmungswinkels (Cobb-

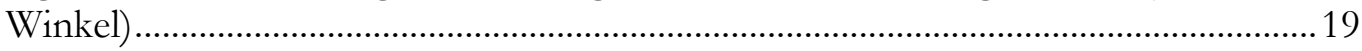

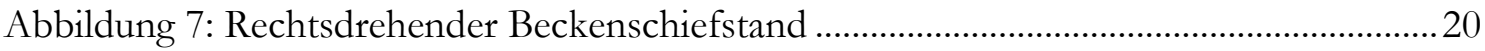

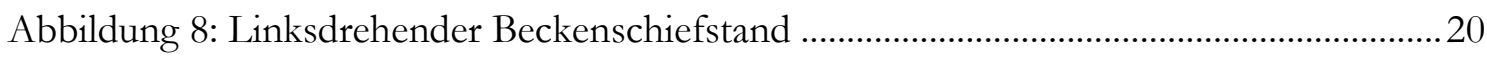

Abbildung 9: Schemazeichnung zur Messung im sagittalen Profil ..........................................2 23

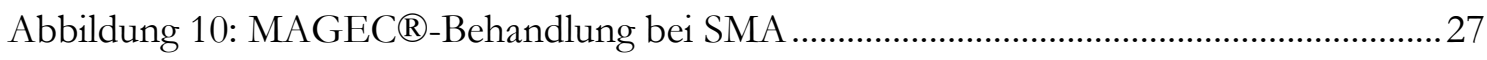

Abbildung 11: Skoliosekrümmungswinkel (Cobb-Winkel) des Gesamtkollektivs ( $\mathrm{n}=21)$... 32

Abbildung 12: Skoliosekrümmungswinkel gruppiert nach Alter............................................... 33

Abbildung 13: Skoliosekrümmungswinkel gruppiert nach BMI ................................................. 34

Abbildung 14: Beckenschiefstand (pelvic obliquity) des Gesamtkollektivs ( $\mathrm{n}=21)$..................... 36

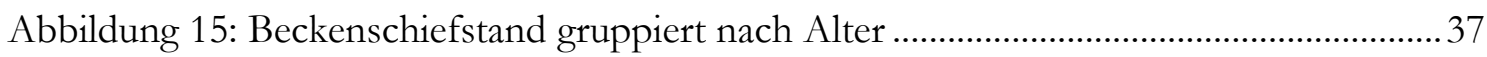

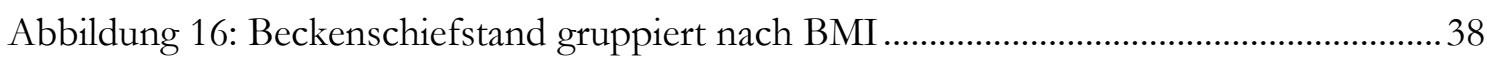

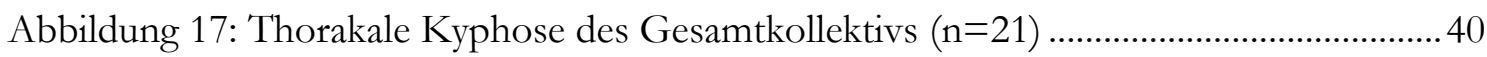

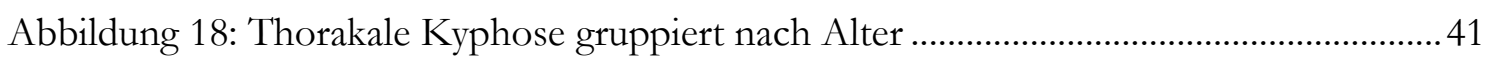

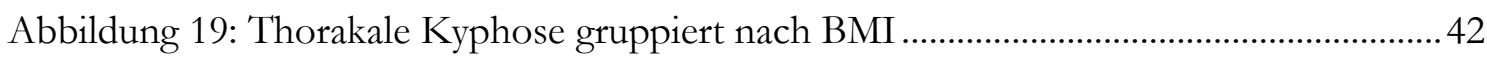

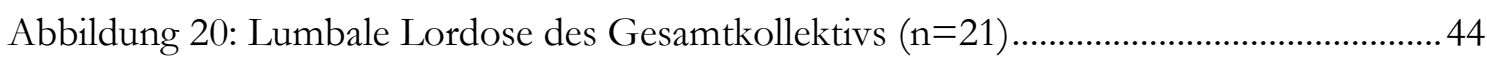

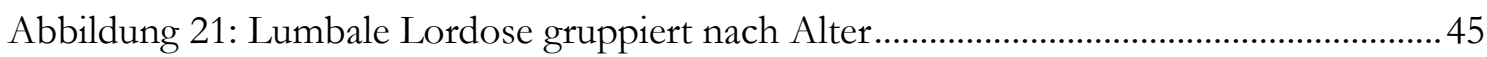

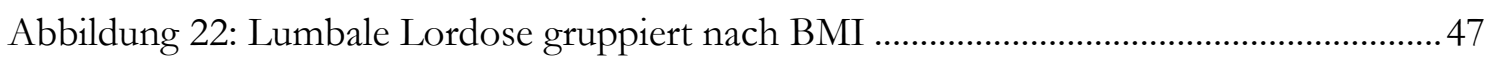

Abbildung 23: Wirbelsäulenlänge (spinal length) des Gesamtkollektivs ( $\mathrm{n}=21)$........................... 48

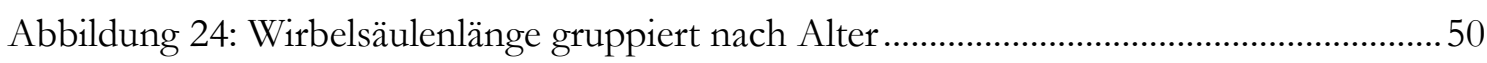

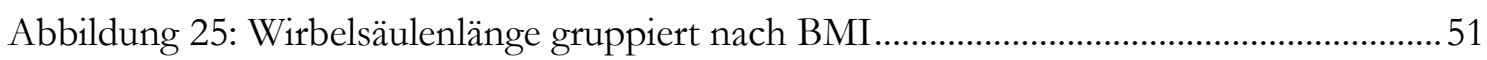

Abbildung 26: Varianz zwischen den Untersuchern anhand des

Skoliosekrümmungswinkels (Cobb-Winkel) …………................................................ 52

\section{Tabellenverzeichnis}

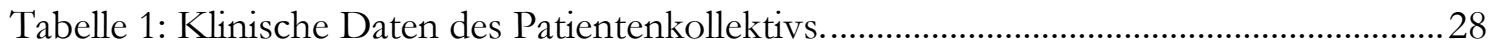




\section{Abkürzungsverzeichnis}

a. p. anterior-posterior

BMI Body-Mass-Index

BWS Brustwirbelsäule

et al. et alii

lat. lateral

L Lendenwirbelkörper

LWS Lendenwirbelsäule

MAGEC® Magnetic Expansion Control

MCGR magnetically controlled growing rod

PJK proximaljunctional kyphosis

SMA Spinale Muskelatrophie

SMN-Gen survival motor neuron-Gen

Th Brustwirbelkörper

TIS Thoraxinsuffizienz-Syndrom

UMG Universitätsmedizin Göttingen

V Verlängerung

VEPTR ${ }^{\circledR} \quad$ vertical expandable prosthetic titanium rib 


\section{Einleitung}

Eine Skoliose ist eine Deformität der Wirbelsäule, die sich in allen drei Körperebenen wiederfindet (Trobisch et al. 2010). Sie lässt sich auf unterschiedliche Weise klassifizieren. So ist eine Einteilung nach Manifestationsalter möglich, aber auch nach Ätiologie oder Form der Krümmung (Trobisch et al. 2010).

Diese Arbeit untersucht eine besondere Form der neuromyopathischen Skoliose, die im Rahmen der spinalen Muskelatrophie (SMA) auftritt. Die SMA ist eine schwerwiegende Erkrankung mit Muskelschwund, die durch einen fortschreitenden Untergang von motorischen Zellen im Vorderhorn des Rückenmarks gekennzeichnet ist. Neben anderen Problemen steht die Entwicklung einer progredienten Skoliose in jungen Jahren im Vordergrund. In dieser Arbeit wird der Einfluss von extern zu kontrollierenden magnetischen Implantaten auf die kindliche Wirbelsäulendeformität bei SMA untersucht.

\subsection{Gängige Klassifikationen der Skoliose}

Von der infantilen Skoliose wird gesprochen, wenn eine Manifestation vor dem vierten Lebensjahr erfolgt (Trobisch et al. 2010). Diese Einteilung, die noch durch eine juvenile (drei bis neun Jahre) und adoleszente Skoliose (zehn bis 18 Jahre) ergänzt wird, orientiert sich zudem am Wachstumsmuster der Wirbelsäule. Es wird angenommen, dass das Wirbelsäulenwachstum in drei unterschiedliche Phasen mit schubweisem Wachstum eingeteilt werden kann, die ungefähr den genannten Zeiträumen entsprechen (Trobisch et al. 2010).

Aus einer weiteren Einteilung nach Alter und gleichzeitig der Prognose, unabhängig von der Ätiologie, resultiert die Bezeichnung early-onset-Skoliose (Gillingham et al. 2006). Sie ist definiert als eine vor dem fünften Lebensjahr auftretende Skoliose (Gillingham et al. 2006). Hier wird mit einbezogen, dass einige Autoren vermuten (Sanders 2007), dass im Alter von drei bis neun Jahren entgegen bisherigen Annahmen kein schubweises Wirbelsäulenwachstum stattfindet. So wird die early-onset-Skoliose der late-onset-Skoliose gegenübergestellt, die sich erst nach dem fünften Lebensjahr manifestiert und als prägnanten Unterschied beinhaltet, dass das Risiko für kardiopulmonale Probleme als Folge der gravierenden Skoliose hier nicht mehr so stark gegeben ist (Gillingham et al. 2006). Im Allgemeinen ist die Prognose der early-onsetSkoliosen deutlich schlechter als die der late-onset-Skoliosen. 
Die idiopathische Skoliose stellt eine Ausschlussdiagnose dar und kann weiter abhängig vom Alter in eine infantile, juvenile, adoleszente oder adulte idiopathische Skoliose unterteilt werden (Trobisch et al. 2010), wovon die adoleszente idiopathische Skoliose (AIS) unter allen Skoliosen unterschiedlicher Ätiologie am häufigsten vorkommt (Crijns et al. 2017).

Die sehr seltene kongenitale Skoliose hat bezüglich der Prognose und der Klinik ein heterogenes Erscheinungsbild. So finden sich hier sowohl schwere Wirbelsäulendeformitäten aufgrund von Fehlbildungen oder Fehlanlagen mit starker Beeinträchtigung der Lunge als auch schwache Skoliosen mit mildem Verlauf (Farley et al. 2014). Zugrunde liegen diesen Skoliosen immer Wirbelkörperdeformitäten, welche als Formationsstörungen, Segmentationsstörungen oder komplexe Mischformen häufig in Kombination mit weiteren Fehlbildungen (z. B. Niere, Lunge, Rippen) während der Schwangerschaft entstehen (Hedequist und Emans 2004).

Die neuromuskuläre Skoliose ist eine generelle Bezeichnung für eine Wirbelsäulenverkrümmung, die auf ein Problem des Nervensystems oder der Muskulatur zurückzuführen ist (Carstens 1999). So können verschiedene Grunderkrankungen eine neuromuskuläre Skoliose hervorrufen (Rumalla et al. 2016). Neurogene Ursachen können z. B. die infantile Zerebralparese, Muskeldystrophien (z. B. Morbus Duchenne), eine Myelomeningozele oder, als Vertreterin der Erkrankungen des zweiten Motoneurons, die SMA (Carstens 1999), sein. Charakteristisch für eine neuromuskuläre Skoliose ist eine großbogige C-förmige, meist thorakolumbale Skoliose, die mit einem Beckenschiefstand (pelvic obliquity) einhergeht (Putzier et al. 2016). Letzteres wird durch eine begleitende einseitige Hüftluxation begünstigt. Beim Vorliegen einer SMA entsteht in 70 bis 100\% eine Skoliose (Canavese et al. 2014; Chandran et al. 2011; Fujak et al. 2013).

Bei sich schnell verschlechternden schwerwiegenden Skoliosen unterschiedlichster Ätiologie können auch die Organe beeinträchtigt werden (Rumalla et al. 2016). Hierbei ist insbesondere die Lunge zu nennen, da sie aufgrund der thorakalen Skoliose nicht genügend Raum hat, um sich entsprechend zu entwickeln, was dann in der Folge zu einem ThoraxinsuffizienzSyndrom (TIS) mit frühzeitigem fatalem Lungenversagen führen kann (Campbell et al. 2003; Akbarnia et al. 2011). Aber auch der massive Beckenschiefstand mit einem Eintauchen des Beckens in den Brustkorb und Hautulzerationen kann massive Probleme verursachen (Brunner und Gebhard 2002). 


\subsection{Spinale Muskelatrophie}

In der vorliegenden Studie wurden ausschließlich Kinder mit der Erkrankung Spinale Muskelatrophie (SMA) untersucht. Die SMA ist definiert als eine hauptsächlich autosomalrezessiv vererbte Erkrankung, die durch einen Defekt im SMN1-Gen hervorgerufen wird (Lefebvre et al. 1995; Russman 2007). Die SMA präsentiert sich als eine Erkrankung des zweiten, im Vorderhorn des Rückenmarks sitzenden, Motoneurons (Russman 2007).

Epidemiologisch handelt es sich hierbei um eine nicht-entzündliche neurodegenerative Erkrankung mit einer Inzidenz von 1:10.000 unter den Neugeborenen (Ludolph 2015). Wie Roberts et al. bereits 1970 beschrieben, trägt die Inzidenz und eine geschätzte Heterozygotenfrequenz von 1:50 (Ziemann 2011) dazu bei, dass die SMA zu einer der häufigsten hereditären autosomal-rezessiven Erkrankungen nach der Mukoviszidose gehört. Die SMA wurde bereits 1995 durch Lefebvre et al. maßgeblich auf eine Mutation im SMNGen (survival motor neuron gene) zurückgeführt, wodurch weniger SMN-Protein gebildet wird. Ferner wurde festgestellt, dass der Phänotyp schwerwiegender ist, je weniger Kopien des SMN-Gens vorhanden sind (Lefebvre et al. 1997; Arakawa et al. 2016). Bei dem Verdacht auf SMA und erhöhten Serumwerten für Kreatinkinase sollte daher eine molekulargenetische Diagnostik erfolgen (Mercuri et al. 2012). Gibt diese kein eindeutiges Ergebnis, besteht die Möglichkeit weiterführender elektrophysiologischer Tests (Mercuri et al. 2012).

Die SMA zählt zu den proximalen spinalen Muskelatrophien (Fujak et al. 2013) und wird im Allgemeinen entsprechend dem Manifestationsalter ihrer typischen Symptome klassifiziert (Forst et al. 2003). Diese sind atrophische Paresen mit dem Erscheinungsbild einer muskulären Hypotonie und erloschenen Muskeleigenreflexen, generalisierter Muskelschwäche, Kontrakturen (Fujak et al. 2012) und in den schwerwiegenden Fällen zu nahe 100\% eine Skoliose (Chandran et al. 2011). Diese ist jedoch, im Gegensatz zu der idiopathischen Skoliose, die selten mit Schmerzen einhergeht (Trobisch et al. 2010), häufig mit Rückenschmerzen assoziiert (Chandran et al. 2011).

\subsubsection{Formen der SMA}

Die aktuelle Klassifikation orientiert sich nach wie vor an der ursprünglichen Einteilung von 1961 durch Byers und Banker, die die spinale Muskelatrophie nach Eintritt ihrer Symptome in vier Typen einteilten. Des Weiteren findet sich in dieser Einteilung auch eine Angabe über die motorischen Funktionen und Lebenserwartung der erkrankten Kinder wieder. Diese ursprüngliche Einteilung wurde durch Untergruppen erweitert (Forst et al. 2003). Da bereits von Lefebvre et al. 1997 herausgefunden wurde, dass der Phänotyp mit abnehmender Anzahl 
der SMN-Genkopien in der Ausprägung der Grunderkrankung gravierender wird, ist nach Russman (2007) für die Zukunft denkbar, die Untertypen der SMA an der Molekulargenetik zu orientieren. Durch die aktuell eingeführte medikamentöse Therapie (Biogen 2017), wird diese Art der Klassifikation in den nächsten Jahren voranschreiten.

Die SMA Typ I ist mit einer Häufigkeit von 50\% die häufigste Form der spinalen Muskelatrophie und gleichzeitig die am schwerwiegendsten verlaufende (Lunn und Wang 2008). Die Symptome treten bei dieser auch als Werdnig-Hoffmann bezeichneten Form der SMA vor dem sechsten Lebensmonat auf (Russman 2007). Nach heutigen Erkenntnissen unterscheiden sich die Unterformen Typ Ia und Ib hinsichtlich der Lebenserwartung, die bei Typ Ia unter zweieinhalb Jahren liegt, bei Typ Ib hingegen bis ins junge Erwachsenenalter reichen kann (Haaker und Fujak 2013). Diese Prognosen werden sich aber bei den heutigen medikamentösen Möglichkeiten verändern (Finkel et al. 2016).

Nach Ludolph (2005) ist es auch möglich, dass diese Krankheit pränatal durch spärliche oder nicht vorhandene Kindsbewegungen in Erscheinung tritt oder sich das Neugeborene postnatal als floppy infant präsentiert (Forst et al. 2003). Sie fallen in der Folge durch einen erniedrigten Muskeltonus und Trinkschwäche auf. Die ersten Paresen finden sich häufig im Beckengürtel wieder (Ludolph 2015).

Durch den außerordentlich gravierenden Verlauf dieser Form und die damit einhergehende schwere muskuläre Schwäche ist es den Kindern zu Lebzeiten nicht möglich, ohne Hilfe aufrecht zu sitzen (Lunn und Wang 2008). Von einer bulbären Beteiligung bzw. Bulbärparalyse spricht man, wenn die Hirnnervenkerne der Medulla oblongata, einem Teil des Hirnstamms, der Reflexe bezüglich des Schluckakts, der Sprache, von Husten oder der Atmung steuert, von einem Niedergang betroffen sind (Ulfig 2008). Dadurch steigt die Aspirationswahrscheinlichkeit, und die darauffolgende Komplikation in Form einer Pneumonie konnte in der Vergangenheit häufig als Todesursache gefunden werden (Lunn und Wang 2008). Ursächlich für die potentiell lebensbedrohliche bzw. limitierende Pneumonie ist ebenfalls, dass im Rahmen der generalisierten Muskelschwäche und der damit ebenfalls einhergehenden Schwäche der Interkostalmuskulatur eine beeinträchtigte Atemmechanik entsteht, die das Entstehen von Atelektasen begünstigt (Ludolph 2015). Durch Fortschritte in der Beatmungstherapie - in Form von nicht-invasiver Beatmung oder invasiver Beatmung und Tracheostomaanlagen - ist die Lebenserwartung dieser Kinder heutzutage angestiegen (Markowitz et al. 2012), was insbesondere die klinische Abgrenzung zum Typ II der SMA erschwert. Bedingt durch die Therapie mit Nusinersen könnten in den kommenden Jahren diese Kriterien zunehmend in den Hintergrund treten. 
Zwischen den beiden Formen der spinalen Muskelatrophie, Typ I und Typ III, findet sich die intermediäre Form, Typ II, wieder. Sie manifestiert sich meist im Alter von sieben bis 18 Monaten (Lunn und Wang 2008). An dieser Form erkrankte Kinder können in der Regel frei sitzen, jedoch lernen sie nicht eigenständig zu laufen, wodurch sie rollstuhlpflichtig sind (Forst et al. 2003). Sie können ebenfalls infolge einer generellen Schwäche der Muskulatur und bei einer eventuellen bulbären Beteiligung Probleme haben zu schlucken oder zu husten, wodurch ihre Lunge aufgrund von Aspiration gefährdet ist (Lunn und Wang 2008). Historische Studien haben ergeben, dass Pneumonien und restriktive Lungenerkrankungen ein häufiger Grund waren, weshalb die Lebenserwartung dieser Patienten oft nicht über 30 Jahre betrug (Markowitz et al. 2012). Durch die aktuell bessere medizinische Versorgung mit Beatmungsmöglichkeiten und der aktuell zugelassenen medikamentösen Therapie mit Nusinersen (Biogen 2017) ist die Lebenserwartung ebenso wie bei SMA Typ I voraussichtlich nicht mehr derart eingeschränkt (Mercuri et al. 2012).

Die juvenile Form der SMA, Typ III, die auch Kugelberg-Welander genannt wird, hat ein sehr heterogenes Erscheinungsbild (Lunn und Wang 2008). Das Manifestationsalter liegt vor dem dritten (Typ III a) oder zwischen dem dritten und 18. Lebensjahr (Typ III b) (Forst et al. 2008). Die motorischen Funktionen können unterschiedlich gut ausgeprägt sein (Lunn und Wang 2008). So wird es ebenso Patienten geben, die eigenständig laufen können, als auch jene, die in ihrer Kindheit verschiedene Formen der Unterstützung ihrer Motorik benötigen. In einer Studie von Evans et al. (1981) wurde gezeigt, dass eine Skoliose bei diesem Typ nicht regelhaft zu finden war und nach Russman et al. (1992) hatte diese, falls vorhanden, keine Tendenz, sich mit zunehmendem Alter zu verschlechtern.

Die adulte Form der SMA tritt meist erst nach dem 20. Lebensjahr in Erscheinung und ist durch rumpfnahe Paresen und einen milden Verlauf geprägt (Ziemann 2011). Wie auch bei der SMA Typ III ist hier die Lebenserwartung nicht eingeschränkt (Russman 2007). Im Gegensatz zu dem autosomal-rezessiven Erbgang der früher auftretenden Formen SMA Typ I-III, wird bei der adulten Form in zwei Drittel der Fälle ein autosomal-dominanter Erbgang beobachtet (Ludolph 2005), der allenfalls mit einer geringfügig verkürzten Lebenserwartung einhergeht.

\subsubsection{Behandlung der SMA}

Nachdem es jahrzehntelang keine kausale Therapie der SMA gab (Lunn und Wang 2008), wurde 2011 erstmals mit Nusinersen eine kausale Therapie im Rahmen von Studien angewandt (Maharshi und Hasan 2017; Biogen 2017). Dieses Medikament setzt am Ursprung 
der SMA an. Nusinersen, mit Handelsnamen Spinraza ${ }^{\circledR}$, steigert die Bildung des SMNProteins (Kirschner et al. 2016), dessen Mangel ursächlich für die Symptome der SMA ist (s.o.) Erste Studien haben für SMA Typ I ein Ansprechen auf das Medikament gezeigt, das sich beispielsweise in Form von erzielten motorischen Meilensteinen äußerte (Finkel et al. 2016). Seit dem 01.06.2017 ist die Therapie in Deutschland zugelassen (Biogen 2017). Aktuell werden Patienten aller Altersgruppen therapeutisch behandelt. Das Behandlungsschema sieht eine intrathekale Medikamentengabe mit Nusinersen mit anschließender Erhaltungstherapie vor. Zur Analyse der Ergebnisse werden standardisierte Protokolle inklusive physiotherapeutischer Untersuchungen zum Muskelstatus durchgeführt (Finkel et al. 2016). Durch diese neuartige Therapie bestehen aber auch viele ungelöste Fragen in Form von Applikation des Medikamentes bei schweren Skoliosen oder nach Wirbelsäulenoperation.

Unabhängig von der neuen medikamentösen Therapie wird bei fortgeschrittener Erkrankung versucht, die verschiedenen Erscheinungen dieser Erkrankung symptomatisch zu behandeln und ihre Auswirkungen herabzumindern.

Den Lungen kommt bei an SMA erkrankten Kindern besondere Aufmerksamkeit zuteil. Die durch die generalisierte Muskelschwäche beeinträchtigte Atmung kann zunächst durch eine Nachtbeatmung unterstützt werden, die in der Folge auf eine ganztägige nicht-invasive Beatmung erweitert werden kann (Mercuri et al. 2012). Bei Zunahme der respiratorischen Insuffizienz kann die Anlage eines Tracheostomas erforderlich werden (Castro und Iannaccone 2014). Regelmäßige Lungenfunktionsprüfungen, die von einer pulsoxymetrischen Messung, bei Bedarf auch bei Nacht, begleitet werden können (Mercuri et al. 2012), müssen unbedingt erfolgen. In einer Studie fanden Fujak et al. (2013) heraus, dass die Lungenfunktion von Kindern mit SMA Typ II und IIIa sich mit zunehmendem Alter in der Wachstumsphase verschlechterte (Fujak et al. 2013). Darüber hinaus können die Kinder Schutzreflexe, wie Husten, aufgrund ihrer Muskelschwäche und einer bereits erläuterten potentiellen bulbären Beteiligung (Lunn und Wang 2008) nicht ausreichend nutzen. Zum besseren Abhusten des sich ansammelnden Sekrets, welches durch den verminderten Hustenstoß entsteht, ist eine Unterstützung in Form eines cough assist nötig (Mercuri et al. 2012; Haaker und Fujak 2013). Der cough assist ist eine Maschine, die mittels Aufbau eines positiven Drucks eine nicht-invasive Entfernung des Sekrets aus den Lungen ermöglicht.

Durch das vermehrte Sekret und den Sekretstau besteht außerdem eine erhöhte pulmonale Infektionsgefahr (Haaker und Fujak 2013), die mit Impfungen gegen Influenza und RSV (respiratory syncytial virus) gesenkt werden konnte (Castro und Iannaccone 2014). Zur 
Verbesserung der Lungenfunktion ist weiterhin eine regelmäßige Krankengymnastik mit Atemtherapie sinnvoll (Mercuri et al. 2012).

Hinsichtlich des Bewegungsapparats sind im Rahmen einer SMA Kontrakturen der großen Gelenke (Hüften, Knie, Obere Sprunggelenk, Schulter, Ellbogen) zu erwarten, denen mit Bewegungs- und Stehtraining und Orthesen entgegengewirkt werden kann (Castro und Iannaccone 2014). Häufig verlaufen diese Versuche jedoch frustran. Ein weiteres Problem ist die Instabilität in der Sitzposition, die in erheblichem Maße negativ vom Beckenschiefstand beeinflusst wird (Fujak et al. 2013). Hüftluxationen sind häufig (Putzier et al. 2016) und bedürfen bei fehlenden Beschwerden keiner operativen Therapie (Sporer und Smith 2003).

Aus der hochgradigen körperlichen Behinderung resultieren Folgeprobleme wie eine Osteoporose (Vai et al. 2015), welche als Folge einer mangelnden Knochenbelastung und der Rollstuhlpflichtigkeit auftritt. Zur Behandlung und Vorbeugung dieser Problematik und zur Verbesserung von Kontrakturen hat sich die Galileotherapie (Vry et al. 2014), eine Ganzkörpervibrationstherapie, in den letzten Jahren besonders für SMA als günstig erwiesen. Eventuell sind auch medikamentöse Behandlungen notwendig.

Ein großes Problem für operative therapeutische Maßnahmen stellt die Kachexie dar. Diese entsteht aufgrund der bulbär bedingten Schluckprobleme in dieser Patientengruppe und ist trotz hochkalorischer Nahrungszufuhr oder Anlage einer Magensonde nur schwer zu behandeln (Burnett et al. 2009).

\subsection{Behandlung der Skoliose bei SMA}

In einer Studie bestätigten Fujak et al. (2013) das Ergebnis von Granata et al. (1989), dass der Skoliosekrümmungswinkel von Kindern, die an SMA Typ II leiden, pro Jahr um acht Grad zunahm. Diese bei SMA auftretende early-onset-Skoliose entwickelte sich früh im Alter unter fünf Jahren und verschlechterte sich bei ausbleibenden therapeutischen Maßnahmen rasant (Granata et al. 1989; s. Abbildung 1).

Die Skoliose führt nicht nur zu einer Seitausbiegung der Wirbelsäule, sondern auch zu einer Rotation. Dadurch kommt es zu einer Verbiegung der Rippen, die zum einen mit einer gestörten Rippenmechanik und zum anderen mit einer Thoraxdeformität einhergeht. Dieser dysplastische Thorax resultiert in Kombination mit einer gestörten Rippenmechanik in einem verminderten Thoraxvolumen. Dies führt bei Skoliosepatienten mit einer SMA durch die zunehmende Muskelschwäche der Atem-, Bauch- und Zwerchfellmuskulatur zu einer Verschlechterung der Lungenfunktion. Eine Korsettherapie zur konservativen Behandlung 
einer Skoliose ist deshalb in den allermeisten Fällen aufgrund der insuffizienten Atmung kontraindiziert (Sucato 2007).
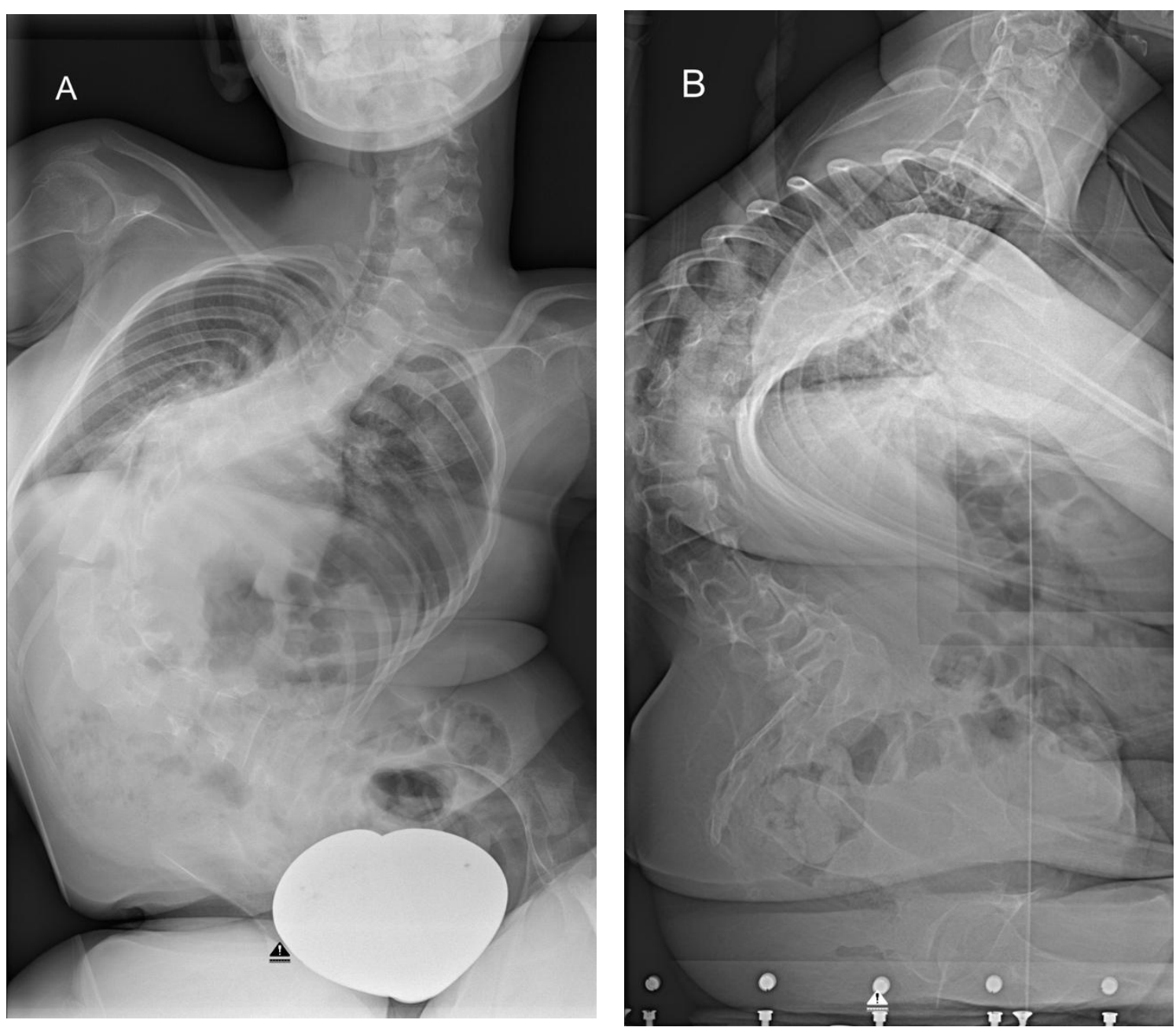

Abbildung 1: Röntgenaufnahmen eines 13-jährigen Kindes mit konservativ behandelter Skoliose bei SMA Typ II. Die a. p.-(A) und laterale (B) Röntgenaufnahme zeigen eine nicht operativ behandelte rechtskonvexe thorakale Skoliose mit einem Skoliosekrümmungswinkel von $138^{\circ}$. (Röntgenaufnahme einer Patientin der Kinderorthopädie der UMG, 2017).

Durch die zumeist beträchtliche Thoraxdeformität im Rahmen der Skoliose können die Lungen in ihrer Expansion gestört sein (Gillingham et al. 2006). Eine Einschränkung des Lungenwachstums resultiert in einer verminderten Alveolenanzahl (Akbarnia et al. 2005), die zu einer respiratorischen Insuffizienz führt. Folglich kann bei Kindern mit early-onset-Skoliose ein Thoraxinsuffizienz-Syndrom (TIS) bestehen (Campbell et al. 2003). Dieses Syndrom beschreibt die Unfähigkeit des Brustkorbes, Platz für eine physiologische Atmung oder ein physiologisches Lungenwachstum zu gewährleisten (Campbell et al. 2004b). Diagnostische Kriterien für ein TIS sind nach Campbell et al. (2003) klinische Zeichen einer Ateminsuffizienz, eine verringerte Beweglichkeit des Brustkorbs und Auffälligkeiten in der Lungenfunktion. Das TIS begünstigt des Weiteren eine fehlende altersgerechte 
Gewichtszunahme aufgrund eines erhöhten Grundumsatzes wegen vermehrter Atemarbeit (Bowen et al. 2008).

Die Skoliosebehandlung hat heute zum Ziel, nicht bloß ein weitestgehend normales Wirbelsäulenwachstum zu ermöglichen, sondern auch eine Thoraxsymmetrie wiederherzustellen und Volumen $\mathrm{zu}$ vergrößern und somit die Lungenentwicklung $\mathrm{zu}$ verbessern (Campbell et al. 2003; Gomez et al. 2011). Ehemals stand das Problem der knöchernen Deformität der Wirbelsäule im Vordergrund, welches mit einer Versteifung frühzeitig angegangen wurde (Vitale et al. 2008) und letztendlich häufig bei kleinem deformierten Thorax in einer respiratorischen Insuffizienz mit verkürzter Lebenserwartung mündete (Hell et al. 2005; Vitale et al. 2008).

\subsubsection{Operative Verfahren und Implantate}

Da Kinder mit SMA frühzeitig eine rasant progrediente Skoliose entwickeln und wie oben erläutert eine konservative Therapie nicht zielführend ist, wurden schon frühzeitig sogenannte „mitwachsende“ Systeme zur operativen Aufrichtung der Wirbelsäulendeformitäten entwickelt (Granata et al. 1989). Heutzutage wird die Korrektur von Wirbelsäulen- und Thoraxdeformitäten zur Vermeidung eines TIS schon frühzeitig mit growth friendly spinal implant-Systemen durchgeführt. Hier stehen verschiedene Systeme, z. B. growing rods, vertical expandable prosthetic titanium rib (VEPTR $®)$ - oder magnetic expansion control (MAGEC () )Implantate und andere zur Verfügung (Campbell et al. 2004a; Tis et al. 2012; Skaggs et al. 2014).

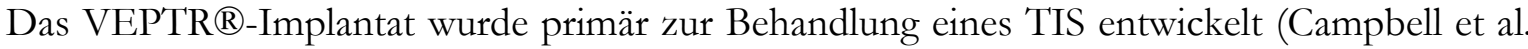
2003; Hell et al. 2005; Mayer und Redding 2009). Bei diesem Implantat handelt es sich um ein uni- oder bilaterales expandierendes Stabsystem, das kranial an den Rippen und kaudal am Beckenkamm oder der Lendenwirbelsäule befestigt wird (Campbell et al. 2004a). Im Unterschied zu einer definitiven Wirbelsäulenversteifung werden halbjährliche operative Verlängerungen des Teleskopstabs des VEPTR ${ }^{\circledR}-S y s t e m s$ durchgeführt zur Generierung von Wirbelsäulenlänge und Wachstum (Hell et al. 2005). Demgegenüber kann nach einer Versteifungsoperation die Wirbelsäule nicht weiterwachsen.

Das auch als Expansionsthorakoplastik bezeichnete Verfahren soll in erster Linie den Thorax aufspannen und vergrößern und sekundär die Skoliose der Wirbelsäule verbessern und somit im Verlauf durch die Verlängerungen ein Wachstum von Wirbelsäule und Thorax generieren (Campbell et al. 2004b). Durch Implantation eines VEPTR®-Systems bei kindlichen 
Wirbelsäulendeformitäten wurden in der Literatur eine Reduktion der Hauptkrümmung zwischen 29\% und 51\% beschrieben (Gantner et al. 2018 36,3\%; Emans et al. 2005 29,1\%; Hasler et al. 2010 29,4\%; Campbell et al. 2007 30,7\% und Samdani et al. 2009 38,1\%; Wimmer et al. $201050,7 \%)$.

Bei der SMA handelt es sich im Allgemeinen um eine sehr flexible Wirbelsäulendeformität. Daher ist eine gute Aufrichtung mit wenig Verankerungspunkten, wie z. B. die bilaterale VEPTR ${ }^{\circledR}$ Implantation mit einer Verankerung zwischen Rippen und Beckenkämmen, der Eiffeltower construction, günstig. Durch das Anheben der herabhängenden Rippen, dem sogenannten „Tannenbaumphänomen“, wird gleichzeitig der Thorax aufgespannt (Campbell und Smith 2007; Haaker und Fujak 2013).

Trotz verschiedener operativer Therapien konnte eine Änderung bzw. Verbesserung der Lungenfunktion bei SMA-Kindern nicht nachgewiesen werden (Mayer und Redding 2009). Nach Chua et al. (2016) lag dies daran, dass die normalerweise auftretende Verschlechterung der Lungenfunktion im Verlauf bei den Ergebnissen nicht berücksichtigt wurde. Aufgrund ihrer langen follow-up-Zeitspanne von 11,6 Jahren im Durchschnitt konnten sie hingegen zeigen, dass die Forcierte Vitalkapazität (FVC) vor der VEPTR ${ }^{\circledR}-$ Implantation von einer jährlichen Abnahme von 5,31\% zu postoperativ 1,77\% jährlich abnahm. Daher konnte neben einer Verbesserung der Lebensqualität nun auch eine Verbesserung der Lungenfunktion bei SMA-Kindern gezeigt werden (Chua et al. 2016).

Bei all diesen Vorteilen ist aber nicht außer Acht zu lassen, dass alle wachstumsfreundlichen Wirbelsäulenoperationsmethoden eine hohe Komplikationsrate habe. Im Fall des VEPTR ${ }^{\circledR}$ Verfahrens sind dies beispielsweise heterotope Ossifikationen und Fusionen im Bereich der Rippen, Implantatwanderung- und Lockerung sowie die Implantatinfektionen zu nennen (Grönefeld 2012; Groenefeld und Hell 2013).

Des Weiteren erfahren die ohnehin beeinträchtigten Lungen der SMA-Kinder eine zusätzliche Belastung durch die regelmäßigen Intubationsnarkosen, die alle sechs Monate für die geplanten Implantatverlängerungen nötig werden. Damit können eventuell Komplikationen einhergehen, wie Verschlechterung der Lungenfunktion oder Aufenthalte auf der Intensivstation (Fujak et al. 2012).

Um diesen Problemen entgegenzuwirken, wurde erstmals an der Universitätsmedizin Göttingen 2011 ein operatives Verfahren entwickelt, dass Fixationsanteile des VEPTR ${ }^{\circledR}$ Systems mit den von extern zu steuernden magnetischen Implantaten (MAGEC®) kombiniert (Hell et a. 2018). Im Rahmen von ambulanten Vorstellungen können nun die Implantate von extern gesteuert verlängert werden. 
Somit können MAGEC@-Implantate mit dem VEPTR®-System zur paravertebralen Fixierung kombiniert oder mittels Pedikelschrauben bzw. Laminahaken direkt mit der Wirbelsäule verbunden werden. Der Nachteil letzterer Verfahren mit einer spinalen Verankerung sind die mehrfach beschriebenen heterotopen Ossifikationen entlang der Wirbelsäule mit einhergehenden spinalen Fusionen, welche die Flexibilität der Wirbelsäule vermindern und die nach Wachstumsabschluss der Wirbelsäule notwendige Versteifung erschwert (Lattig et al. 2012; Zivkovic et al. 2014). Unter Verwendung paravertebraler

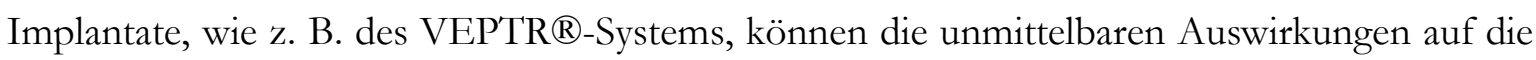
Wirbelsäule und die spinale Fusion vermieden werden (Fletcher und Bruce 2012; Zivkovic et al. 2014).

In dieser Studie wurde ausschließlich die Kombination aus MAGEC@-rods (Firma Ellipse Technologies, Irvine, Inc., California, USA) und VEPTR®-System (Firma DePuy Synthes, Johnson \& Johnson Medical GmbH, deutscher Geschäftsbereich in Umkirch) untersucht. Wie von Hell et al. (2018) beschrieben, liegt der Vorteil in der Kombination einer VEPTR ${ }^{\circledR}$ Verankerung mit MAGEC®-Implantaten zum einen in der Technik, welche nicht die Wirbelsäule an sich berührt und damit eine intrathekale Medikamentenapplikation und eine spätere operative Wirbelsäulenversteifung erleichtert, und zum anderen in der Reduzierung von Mehrfachoperationen. Der Teleskopstab des MAGEC®-Implantats enthält einen Magneten, der bei Auflage eines externen Controllers zum Rotieren gebracht werden kann. Dies hat ein Ausfahren bzw. eine Distraktion des Implantates zur Folge. So wird eine nichtinvasive Verlängerung ermöglicht, wodurch die Komplikationen im Rahmen eines operativen Verfahrens und stationären Aufenthalts eliminiert werden (Cheung et al. 2012; Fujak et al. 2012). Diese perkutane Distraktion dauert eine bis zwei Minuten, ist schmerzfrei und bedarf keinerlei Medikation.

Ebenso wie das VEPTR®-Implantat, soll das MAGEC®-System eine Korrektur der kindlichen Skoliose gewährleisten, solange sich die Wirbelsäule noch im Wachstum befindet. Nach Abschluss des Längenwachstums muss in den allermeisten Fällen eine endgültige Versteifung der Wirbelsäule erfolgen (Thompson et al. 2016). Damit sind growth friendly spinal implant systems sogenannte bridging-Verfahren; sie sind nicht für einen lebenslangen Verbleib im Patienten konzipiert und sollten gegen Ende des Wachstums explantiert werden. In Abhängigkeit der Grunderkrankung des Patienten kann dann die Therapie beendet werden oder es muss eine definitive Spondylodese erfolgen.

Das MCGR (magnetically controlled growing rod)-System wurde einem Kind erstmalig von einem Team der Universitätsklinik Hong Kong um Prof. Kenneth Man-Chee Cheung im November 
2009 implantiert, nachdem das Verfahren bereits in tierischen Studien erprobt worden war (Cheung et al. 2012). Hier wurde eine Fixierung an der Wirbelsäule gewählt. In der Kinderorthopädie der Universitätsmedizin Göttingen (UMG) erfolgte die erste Versorgung eines Kindes mit extern zu expandierenden magnetischen Implantaten im Jahre 2011. Damals wurde ein neuartiges Operationsverfahren entwickelt (Hell et al. 2018), welches eine Rippen $\mathrm{zu}$ Beckenkamm VEPTR ${ }^{\circledR}$-Fixierung mit bilateralen magnetisch zu expandierenden Implantaten kombiniert. Seither wurden in der UMG mehr als 40 solcher Versorgungen bei Kindern durchgeführt. In den letzten Jahren hat sich dieses operative Verfahren besonders für Kinder mit SMA durchgesetzt.

\subsection{Fragestellung der Arbeit}

In der vorliegenden Arbeit wurde folgende Hauptfragestellung untersucht:

Lassen sich flexible Wirbelsäulendeformitäten bei Kindern mit einer spinalen Muskelatrophie durch eine Implantation und wiederholte ambulante Verlängerung von bilateralen extern zu steuernden magnetischen Wirbelsäulenimplantaten während des Wachstums korrigieren und kontrollieren?

Als Nebenfragestellungen wurden folgende Aspekte bearbeitet:

Welche implantat-assoziierten Komplikationen traten im Untersuchungszeitraum auf?

Konnte im Untersuchungszeitraum eine Längenzunahme der Wirbelsäule nachgewiesen werden? 


\section{Material und Methoden}

\subsection{Ethikkommission}

Das Studienprotokoll mit dem Arbeitstitel „Die Behandlung der kindlichen Skoliose bei spinaler Muskelatrophie mit extern zu kontrollierenden magnetischen Implantaten“ wurde durch die Ethikkommission der Medizinischen Fakultät der Georg-August-Universität Göttingen geprüft und am 17.08.2015 mit der Antragsnummer DOK_246_2015 genehmigt.

\subsection{Studiendesign}

Die Studie wurde von Dezember 2011 bis September 2016 als prospektive Kohortenstudie in der Kinderorthopädie (Prof. Dr. Anna-K. Hell) an der Klinik für Unfallchirurgie, Orthopädie und Plastische Chirurgie der Universitätsmedizin Göttingen durchgeführt.

\subsubsection{Ein- und Ausschlusskriterien des Studienkollektivs}

Es wurden Kinder und Jugendliche mit SMA und Skoliose in das Studienkollektiv eingeschlossen, die sich in der Kinderorthopädie der Universitätsmedizin Göttingen wegen einer operationsbedürftigen Wirbelsäulendeformität im Wachstumsalter vorstellten. Entweder erfolgte eine Primärimplantation eines MAGEC®-Systems, oder SMA-Kinder mit einer vorbestehenden bilateralen Rippe-zu-Beckenkamm VEPTR ${ }^{\circledR}-$ Versorgung wurden bei gleichen Verankerungspunkten in MAGEC®-Systeme konvertiert. Als Einschlusskriterium diente weiterhin ein follow-up von mindestens fünf ambulanten Verlängerungsterminen, bzw. von mindestens 17 Monaten Zeit nach Einbau des MAGEC®-Systems.

Ausgeschlossen wurden Kinder mit SMA und Skoliose, welche mittels des MAGEC®Systems operiert wurden, sofern sich der Nachuntersuchungszeitraum auf weniger als fünf Verlängerungstermine oder unter 17 Monate belief.

\subsubsection{Endpunkt der Studie}

Der Endpunkt der Studie wurde anhand von drei Kriterien festgelegt. Zunächst wurde eine Mindestanzahl von fünf Verlängerungsterminen, welche alle drei Monate stattfanden, bestimmt. Ferner endete die Teilnahme eines Patienten an der Studie, wenn ein Ausbau seines Implantats im Rahmen einer Versteifung der Wirbelsäule erfolgte oder aufgrund von 
Komplikationen eine operative Implantatrevision erfolgte. Letzteres Kriterium wurde gewählt, da durch eine erneute Operation im Allgemeinen durch die potentere Distraktion intraoperativ ein Sprung in den Messwerten zu erwarten ist.

\subsection{Patientenkollektiv}

In der vorliegenden Studie wurden die Daten von 21 Kindern mit SMA und neuromyopathischer Wirbelsäulendeformität analysiert, welche mit einem

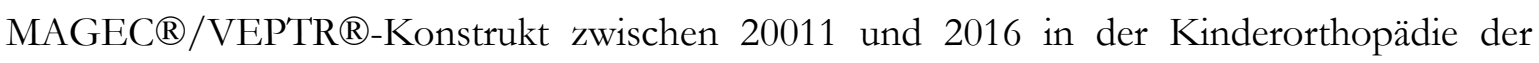
Universitätsmedizin Göttingen operativ versorgt wurden. Aus diesem Kollektiv waren vier Patienten primär operativ mit einem beidseitigen VEPTR ${ }^{\circledR}-S y s t e m$ versorgt worden, welches im Verlauf auf ein MAGEC®-System umgebaut wurde. Die anderen 17 Patienten erfuhren eine Primärimplantation des MAGEC®-Implantats.

\subsubsection{Untersuchungsabläufe}

Die vier Patienten, die zunächst mit einem VEPTR ${ }^{\circledR}$-Implantat versorgt worden waren, erhielten in der Folge einen Umbau auf das MAGEC®-System. Die anderen Patienten wurden primär mit dem MAGEC®-System versorgt. Nach diesem Umbau, bzw. der Primärimplantation, erfolgte die erste ambulante Verlängerung der Implantate in der Regel nach fünf Monaten, anschließend in einem Intervall von drei Monaten.

\subsubsection{Primärimplantation}

Die Implantation der bilateralen MAGEC®-Systeme in Kombination mit einer Rippe-zuBeckenkamm-Fixierung (Hell et al. 2018; Campbell et al. 2004) erfolgte unter Allgemeinanästhesie in Bauchlage.

Die Operation folgt vereinfacht drei Schritten: Zunächst werden auf der konkaven Seite der Krümmung zumeist zwei kraniale Rippen (z. B. Rippen 4-5) mit einem Rippenring des VEPTR ${ }^{\circledR}-S y s t e m s$ gefasst. Für eine bessere Stabilität können optional zwei weitere Rippen mehr lateral mit einem weiteren Implantat fixiert werden. Im zweiten Schritt erfolgt über einen weiteren Hautschnitt das Aufstecken eines Beckenkammhakens auf die Crista iliaca der gleichseitigen Beckenschaufel. Nach Untertunnelung der Weichteile erfolgt das Einbringen des MAGEC®-rods zwischen diese Verankerungspunkte. Dazu werden die Muskulatur und die Weichteile von kranial nach distal mit einer stumpfen Klemme unterfahren, eine Thoraxdrainage als Führungshilfe durch den Weichteiltunnel gezogen und mit Hilfe dieser das 
Implantat sicher durch die Weichteile geführt. Nach der Konnektion des Längsimplantates mit den Verankerungspunkten wird die Gegenseite analog operativ versorgt.

Unmittelbar in der Operation erfolgt ein In-die-Länge-Ziehen der Wirbelsäule durch die Implantate mit einer indirekten Korrektur der Skoliose ohne direkten Kontakt der Implantate mit der Wirbelsäule (Abbildung 2).

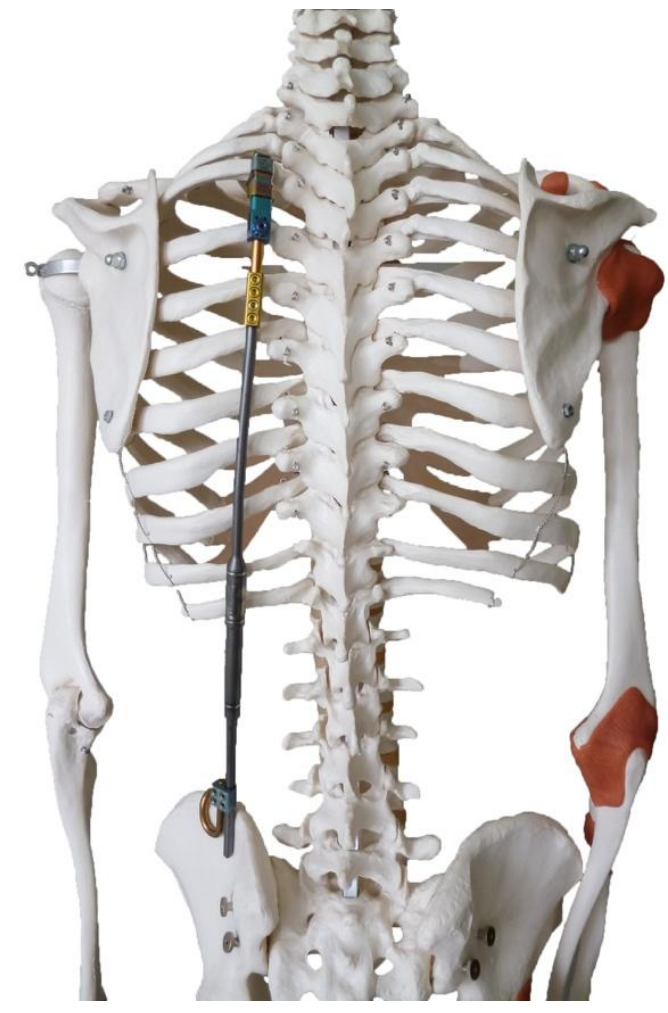

Abbildung 2: Skelett mit Implantation eines MAGEC®-Stabs von dorsal. Zu sehen ist ein Skelett, an dem ein MAGEC $®-S t a b$ (Firma Ellipse Technologies) paravertebral verankert ist. Kranial ist der Stab durch einen VEPTR ${ }^{\circledR}$-Rippenring (Firma Synthes), kaudal durch einen Dunn-McCarthy-Haken am Beckenkamm verankert. (Modell und Fotographie: Arbeitsgruppe der Kinderorthopädie, UMG)

\subsubsection{Verlängerungsprozeduren}

Alle Kinder und ihre Familien wurden präoperativ über das zu erwartende Prozedere detailliert aufgeklärt und bekamen nach der Operation eine schriftliche Mitteilung über den nächsten ambulanten Verlängerungstermin, welcher jeweils in der Röntgenabteilung der Universitätsmedizin Göttingen stattfand. Dort wurden die Kinder vermessen und ihr Gewicht erhoben, um sicherzustellen, dass die Minimalanforderung an ein growth friendly spinal implant system erfüllt wird, nämlich, dass die Kinder unter der Therapie gedeihen. Zu Beginn der Studie wurden sowohl vor als auch nach der Verlängerung sitzende Röntgenbilder der Wirbelsäule in zwei Ebenen erstellt. Somit sollte sichergestellt werden, dass die Implantate zwischen den Verlängerungsterminen nicht an Länge verloren hatten, wie dies von Cheung et al. (2012) für 
extern zu steuernde magnetische Implantate beschrieben worden war. Nachdem dieses Problem im eigenen Krankengut nicht festgestellt werden konnte, erhielten Kinder, die nach Juni 2014 eine Verlängerung erfuhren, zur Reduktion der radiologischen Strahlung nur eine radiologische Untersuchung in zwei Ebenen jeweils nach einer Implantatverlängerung.

Für den Vorgang der Verlängerung legten sich die Kinder in Bauchlage auf die Untersuchungsliege. Durch manuelles Ertasten der Stäbe und mithilfe eines externen Magneten (s. Abbildung 3), MAGEC® Wand Magnet Locator (Ellipse Technologies, Inc., Irvine, California, USA), wurde die Position des Magneten innerhalb des Stabes ermittelt. Dort konnte nun der MAGEC ${ }^{\circledR}$ External Remote Controller (Ellipse Technologies, s. Abbildung 4) aufgesetzt werden und die gewünschte Verlängerung, die in der Regel um die $5 \mathrm{~mm}$ betrug, erfolgte. Durch ein regelmäßiges Schwingen des Magneten im MAGEC® External Remote Controller kann bei diesem Verfahren der Magnet im Stab rotieren, wodurch ein Ausfahren des Stabes möglich wird (Ellipse Technologies 2014). Wie oben beschrieben, erfolgte zur Kontrolle des Verlängerungsvorganges im Anschluss ein sitzendes Röntgenbild der gesamten Wirbelsäule im a. p. (anterior posterior) und lateralen Strahlengang.

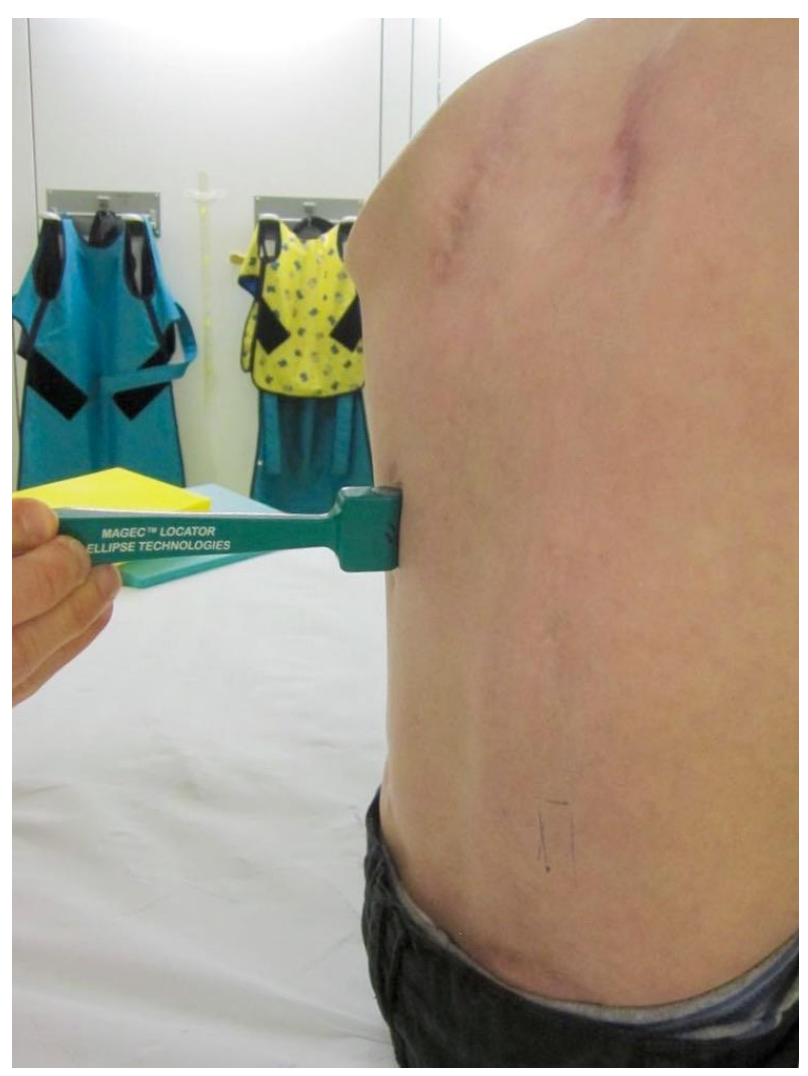

Abbildung 3: MAGEC® Wand Magnet Locator der Firma Ellipse Technologies (Ellipse Technologies, Inc., Irvine, California, USA). Externer Magnet zum Lokalisieren des Magneten im Teleskopstab (Fotographie erstellt von Dr. H. Lorenz, Arbeitsgruppe Kinderorthopädie, UMG, 2017) 


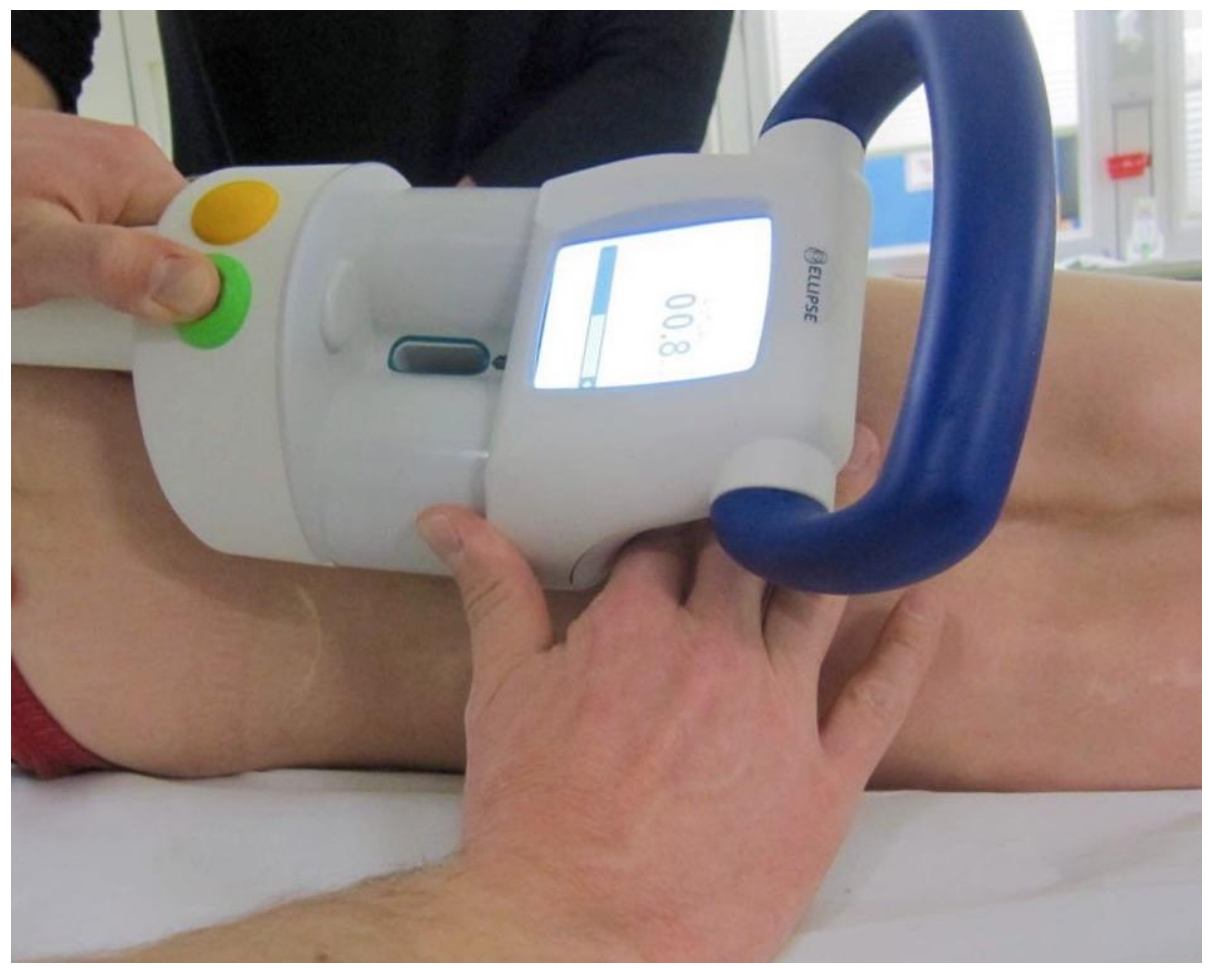

Abbildung 4: MAGEC ${ }^{\circledR}$ External Remote Controller der Firma Ellipse Technologies (Ellipse Technologies, Inc., Irvine, California, USA). (Fotographie erstellt von Dr. H. Lorenz, Arbeitsgruppe Kinderorthopädie, UMG, 2017)

\subsection{Datenerhebung}

\subsubsection{Erfassung der Daten}

Anhand der Einschlusskriterien wurden die untersuchten Patienten identifiziert und in pseudonymisierter Form in einer Excel-Tabelle (Microsoft Office Professional Plus 2010, Firma Microsoft, Redmond, Washington State, Vereinigte Staaten von Amerika) erfasst, die die Grundlage für die Auswertung dieser Studie bildete. Daten wie Geschlecht, Geburtsdatum, Klassifizierung der SMA, Begleiterkrankungen, Mobilität, Komplikationen, Alter bei

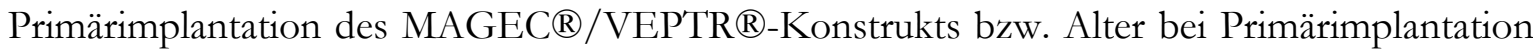
eines VEPTR ${ }^{\circledR}$-Systems und entsprechende vorherige operative Verlängerungsoperationen wurden dokumentiert. Der Body-Mass-Index (BMI) sowie eventuell fehlende Angaben zu den oben genannten Kriterien wurde prospektiv erfasst oder elektronisch (ixserv ${ }^{\circledR}$ Version 4.25, ixmid Software Technologie GmbH, Köln) aus Arztbriefen und Papierakten herausgesucht.

\subsubsection{Radiologische Datenerfassung}

Von allen Patienten wurden vor der Primärimplantation Röntgenbilder der ganzen Wirbelsäule sitzend im a. p. - bzw. p. a. (posterior anterior) - Strahlengang und im seitlichen 
Strahlengang durchgeführt. Hierzu wurden die Kinder aufgrund ihrer Rollstuhlpflichtigkeit in einem speziell gefertigten röntgenstrahlendurchlässigen Stuhl geröntgt (s. Abbildung 5). Dieser Stuhl ist eine Spezialanfertigung des Orthopädie-Mechanikermeisters Stephan Morth (Firma Movimento, Leipziger Str. 359, 34123 Kassel).

Die Auswertung aller Röntgenaufnahmen erfolgte digital im Verarbeitungsprogramm Centricity $^{\mathrm{TM}}$ (Version 3.0, 2006, General Electric Healthcare, Chalfont St. Giles in Buckinghamshire, Großbritannien).

Die Röntgenbilder wurden von zwei Untersuchern unabhängig voneinander ausgewertet und vermessen. Um die Messgenauigkeit zu erhöhen, wurde sich vorher auf die jeweilige Vergrößerung bei jedem zu erhebenden Messwert geeinigt, die in der Folge einheitlich genutzt wurde. Die Grundlage für die Ergebnisse dieser Arbeit bilden die Mittelwerte, die durch die voneinander unabhängigen Ergebnisse der beiden Untersucher ermittelt wurden.

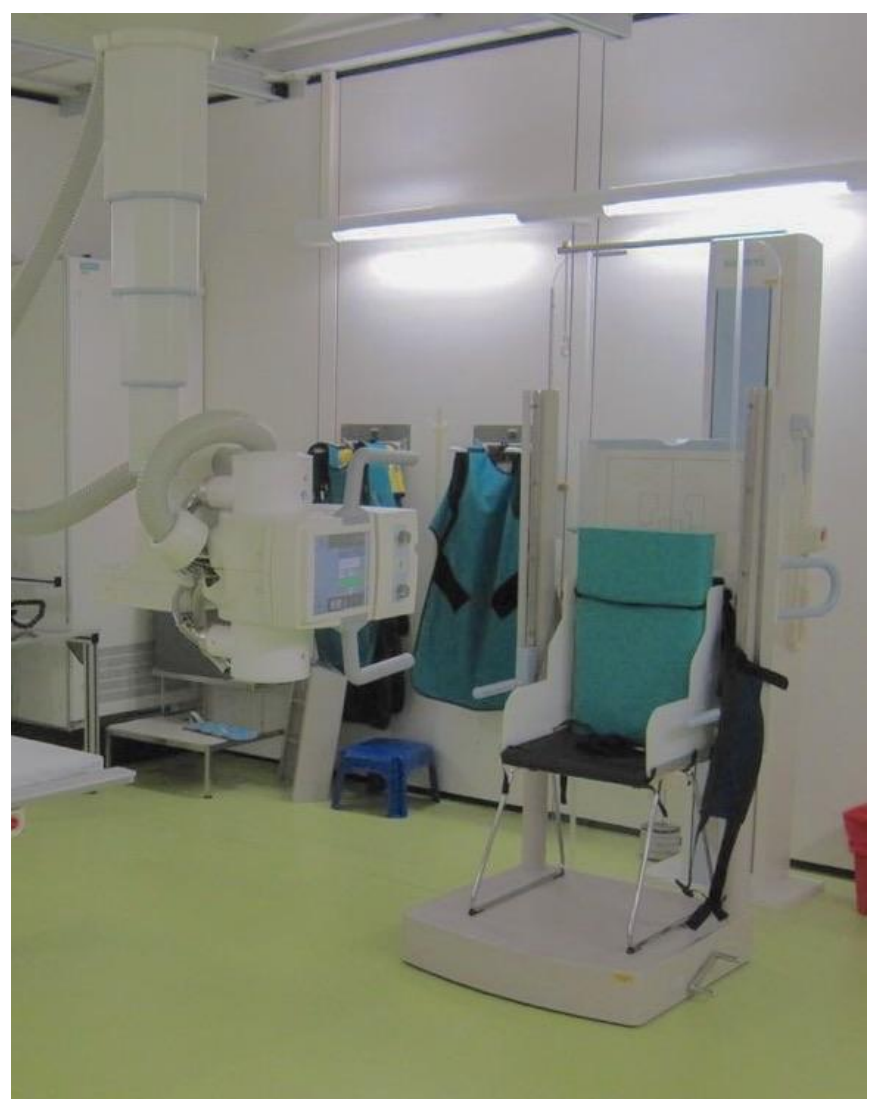

Abbildung 5: Strahlendurchlässiger Stuhl zur Aufnahme von Röntgenbildern. Der Stuhl ist eine Spezialanfertigung des Orthopädie-Mechanikermeisters Stephan Morth Firma Movimento, Kassel. (Fotographie erstellt von Dr. H. Lorenz, Arbeitsgruppe Kinderorthopädie, UMG, 2017)

\subsubsection{Skoliosemessung anhand des Skoliosekrümmungswinkels (Cobb-Winkel)}

Die Wirbelsäulendeformität in der Frontalebene kann mit der Bestimmung des Skoliosekrümmungswinkels quantifiziert werden (Cobb 1948). Gemäß der 
Originalbeschreibung wird im a. p.-Röntgenbild der gesamten Wirbelsäule ein Anfangs- und Endwirbel bestimmt. Diese werden auch als oberer und unterer Neutralwirbel bezeichnet und weisen nicht nur die stärkste Neigung gegen die Horizontale, sondern auch die geringste Rotation auf. An die Grundplatte des kaudalen Neutralwirbels und die Deckplatte des kranialen Neutralwirbels werden Tangenten gelegt. Den Winkel zwischen den Tangenten nennt man nach seinem Beschreiber Cobb-Winkel (Cobb 1948; Abbildung 6). Weil vor Beginn der Studie die Vermutung bestand, dass sich die Hauptkrümmung im Verlauf der Jahre ändern könnte, z. B., dass zu Beginn die lumbale und gegen Ende die thorakale Seitausbiegung die größte Krümmung aufweist, wurde für jeden Patienten anhand des präoperativen Röntgenbildes die Hauptkrümmung als Grundlage für alle nachfolgenden Messungen dieser Studie festgelegt und notiert.

Die Messung des Skoliosekrümmungswinkels erfolgte an den digital vorliegenden Röntgenbildern mit Hilfe einer vierfachen Vergrößerung. Es wurde keine größere Vergrößerung gewählt, weil der Überblick bei der vierfachen Vergrößerung eine genauere Einschätzung der Neigung der Grund- bzw. Deckplatte ermöglichte.

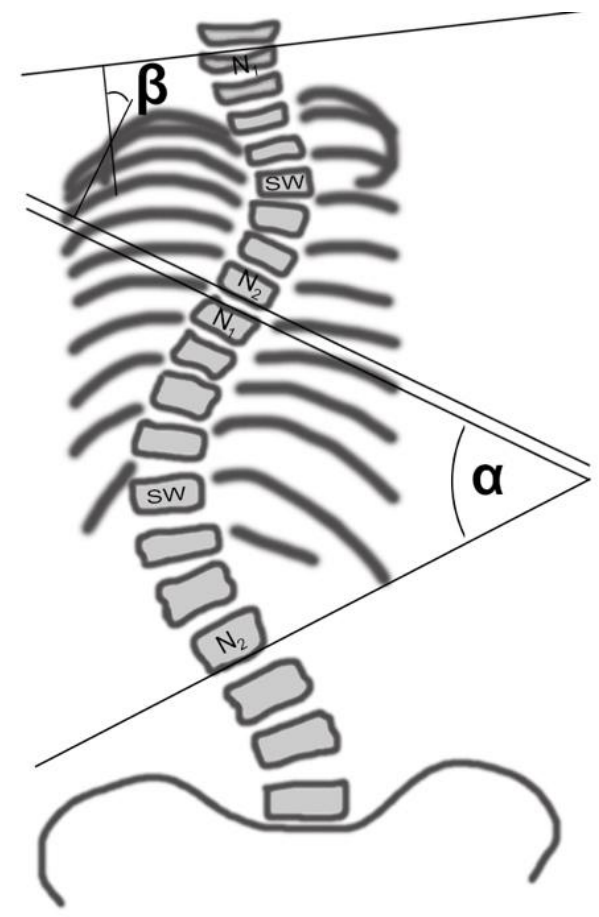

Abbildung 6: Schemazeichnung zur Messung des Skoliosekrümmungswinkels (Cobb-Winkel). Dargestellt ist eine schematische Zeichnung einer a. p.-Röntgenaufnahme mit doppelbogiger Skoliose. Die Neutralwirbel (N1 und N2) und der Scheitelwirbel (SW) sind beschriftet, die Skoliosekrümmungswinkel sind mit $\alpha$ bzw. $\beta$ gekennzeichnet. (Skizze erstellt von Dr. H. Lorenz, Arbeitsgruppe Kinderorthopädie, UMG, 2016) 


\subsubsection{Beckenschiefstand (pelvic obliquity)}

Der Beckenschiefstand (pelvic obliquity) ist ein Maß für die symmetrische Position der Beckenkämme im Stehen oder Sitzen. Im vorliegenden Kollektiv konnte der Beckenschiefstand aufgrund der Rollstuhlpflichtigkeit aller teilnehmenden Kinder lediglich im Sitzen analysiert werden.

Für die Erhebung des Beckenschiefstandes wurde der Winkel zwischen der Horizontalen und der Verbindungslinie der beiden kranialsten Punkte der Beckenkämme gemessen. Um an den Messwerten zu erkennen, ob ein Schiefstand rechts- oder linksdrehend vorlag, wurden aus Patientensicht rechtsdrehende Schiefstände als negative Werte (Abbildung 7) und linksdrehende Schiefstände, also dem Uhrzeigersinn entsprechend, als positive Werte dargestellt (Abbildung 8). Ebenso wie der Skoliosekrümmungswinkel wurde auch der Beckenschiefstand mit einer vierfachen Vergrößerung gemessen.

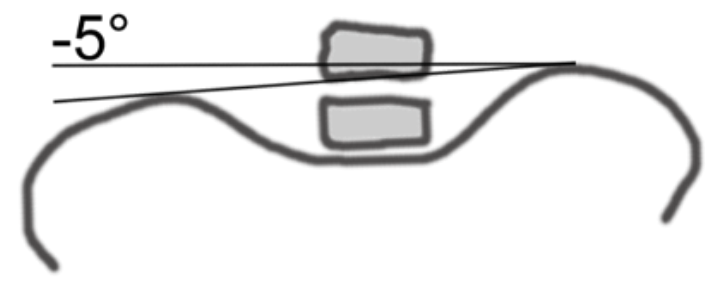

Abbildung 7: Rechtsdrehender Beckenschiefstand. Dargestellt ist eine schematische Zeichnung des Beckenkamms mit Verbindungslinie zwischen beiden höchsten Punkten und dem gemessenen Winkel der rechtsdrehenden Kippung. (Skizze erstellt von Dr. H. Lorenz, Arbeitsgruppe Kinderorthopädie, UMG, 2016)

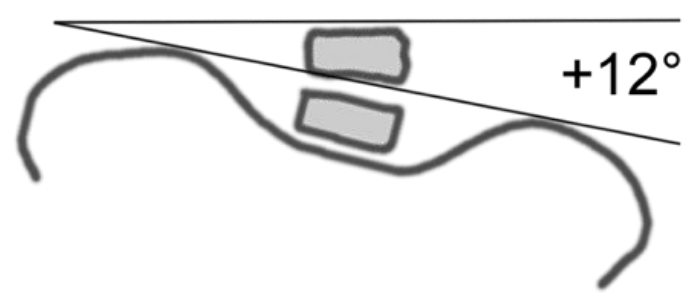

Abbildung 8: Linksdrehender Beckenschiefstand. Dargestellt ist eine schematische Zeichnung des Beckenkamms mit Verbindungslinie zwischen den beiden höchsten Punkten und dem gemessenen Winkel der linksdrehenden Kippung. (Skizze erstellt von Dr. H. Lorenz, Arbeitsgruppe Kinderorthopädie, UMG, 2016) 


\subsubsection{Stabauszug}

Um zu überprüfen, ob der Stab nach der Verlängerung tatsächlich ausgefahren war, wurde der Stabauszug in mm vor und nach der Verlängerung in jeder a. p.-Röntgenaufnahme gemessen. Das duale Stabsystem funktioniert gegenläufig, damit die Ansteuerung der Implantate mit dem Controller einfacher ist und ein unkontrolliertes beidseitiges Ausfahren vermieden werden kann. Für diese Messung wurde zwecks Erreichen der größtmöglichen Präzision eine achtfache Vergrößerung gewählt. Zum Ausschluss eines Vergrößerungs- oder Verkleinerungsfehlers wurde der Stabdurchmesser auf jedem Röntgenbild ausgemessen, welcher bekanntermaßen 9,02 $\mathrm{mm}$ laut Hersteller beträgt (NuVasive, ehemals Ellipse Technologies, Inc., Irvine, California, USA). Wurde ein abweichender Durchmesser ermittelt, so erfolgte eine Anpassung der gemessenen Auszugswerte mittels Dreisatz entsprechend des Vergrößerungsfaktors.

\subsubsection{Thorakale Kyphose und lumbale Lordose}

In der digitalen lateralen Wirbelsäulenganzaufnahme wurden die Krümmungen der Brust- und Lendenwirbelsäule gemessen.

Für die Erfassung dieser Winkel wurden entsprechend des Verfahrens analog der Messung des Skoliosekrümmungswinkels wiederum Tangenten auf die Grund- bzw. Deckplatte der Wirbel gelegt. Der Winkel zwischen diesen Tangenten entspricht der Lendenwirbelsäulen (LWS)Lordose (Winkel $\alpha$ in Abbildung 9) bzw. Brustwirbelsäulen (BWS)-Kyphose (Winkel $\beta$ in Abbildung 9). Bezüglich der LWS-Lordose wurde der kaudale Wirbel immer als Lendenwirbelkörper 5 (L5) definiert. Der kraniale Wirbel hinsichtlich der LWS-Lordose wurde als jener Wirbel definiert, der den Umschlagpunkt zwischen Lordose und Kyphose bildet. War der Umschlagpunkt beispielsweise zwischen L1 und Brustwirbelkörper 12 (Th 12) so bildete L1 den kranialen Wirbel für die Messung der LWS-Lordose und Th12 den kaudalen Wirbel für die Messung der BWS-Kyphose. Um jene wiederum zu erfassen, wurden also der kaudale Wirbel entsprechend des definierten Umschlagpunktes und der zuvor definierte kranialste Wirbel gemessen (Abbildung 9). Zur Standardisierung der Messungen und für einen flüssigeren Arbeitsprozess wurden diese Umschlagspunkte notiert.

Bezüglich der BWS-Kyphose und LWS-Lordose ist nicht außer Acht zu lassen, dass sich diese Werte physiologischerweise während des Alterns verändern (Giglio und Volpon, 2007). So entwickelten Giglio und Volpon 2007 auf Grundlage ihrer Studie bezüglich der Kyphose und Lordose während des Wachstums zwei Formeln, mit denen die Errechnung dieser Winkel im Falle einer gesunden, nicht wirbelsäulendeformierten Person möglich ist. Des Weiteren 
beschrieben Giglio und Volpon (2007), dass es keinen Unterschied zwischen den biologischen Geschlechtern gab, woraufhin eine generelle Formel erstellt wurde.

Es wurde in der Studie dieser Arbeit zunächst der Normwert nach Giglio und Volpon (2007) entsprechend folgender Formel berechnet:

- Normwert der thorakalen Kyphose $=25^{\circ}+0,58 \times$ Alter in Jahren

- Normwert der lumbalen Lordose $=24^{\circ}+0,51 \times$ Alter in Jahren

So wurden für jedes Kind hinsichtlich seines Alters zu jedem Zeitpunkt der radiologischen Untersuchungen die Normwerte für die thorakale Kyphose und die lumbale Lordose errechnet und anschließend die Differenzen zu den gemessenen Werten gebildet. Diese errechneten Werte stellen letztendlich die Abweichung zum altersabhängigen Normwert dar. Sie werden im Folgenden altersadaptierte Kyphose bzw. altersadaptierte Lordose genannt:

- Altersadaptierte Kyphose/Lordose $=$ gemessene Kyphose/Lordose $\mathrm{X}$ altersadaptierter Normwert der Kyphose/ Lordose nach Giglio und Volpon (2007)

Bei positiven Werten besteht eine übermäßige Kyphose oder Lordose, also Hyperkyphose bzw. Hyperlordose; bei negativen Werten herrscht eine geringere Kyphose oder Lordose, also Hypokyphose bzw. Hypolordose.

Folgendes Rechenbeispiel bei einer fiktiv gemessenen Kyphose von einmal $52^{\circ}$ und einmal $15^{\circ}$ bei einem 8,5-jährigen Kind soll obiges Verfahren verdeutlichen:

- Altersadaptierte Kyphose $=52^{\circ}-\left(25^{\circ}+0,58 \times 8,5\right)=22,1^{\circ}$; entsprechend einer Hyperkyphose bzw. 22,1 über dem Altersdurchschnitt

- $\quad$ Altersadaptierte Kyphose $=15^{\circ}-\left(25^{\circ}+0,58 \times 8,5\right)=-14,9^{\circ}$; entsprechend einer Hypokyphyose bzw. $-14,9^{\circ}$ unter dem Altersdurchschnitt 


\subsubsection{Wirbelsäulenlänge}

Das MAGEC®/VEPTR®-Konstrukt soll eine Wirbelsäulendeformitätenkorrektur und eine kontinuierliche Längenzunahme erreichen. Um dies zu objektivieren, wurde die Wirbelsäulenlänge (spinal length) auf der lateralen Wirbelsäulenganzaufnahme ermittelt (Abbildung 9).

Hierfür wurden zwei fixe Messpunkte benötigt, die zuvor definiert worden sind. Der kraniale Messpunkt fand sich somit in der Mitte des thorakalen Wirbelkörpers wieder, der auf Höhe des Rippenhakens zu finden war. Dieser Wirbelkörper wurde in der Microsoft Excel-Tabelle als „kranialster Wirbelkörper“ vermerkt, sodass es in darauffolgenden Messungen nicht zu Verwechslungen kam. Für eine kaudale Begrenzung wurde das Promontorium, der Übergang der Lendenwirbelsäule zum Kreuzbein, aufgesucht und mittels einer Waagerechten markiert. Der Abstand zwischen dem Zentrum des kranialsten Wirbelkörpers und der Waagerechten, welche durchs Promontorium verläuft, entspricht der spinal length (bzw. Strecke d in Abbildung $9)$.

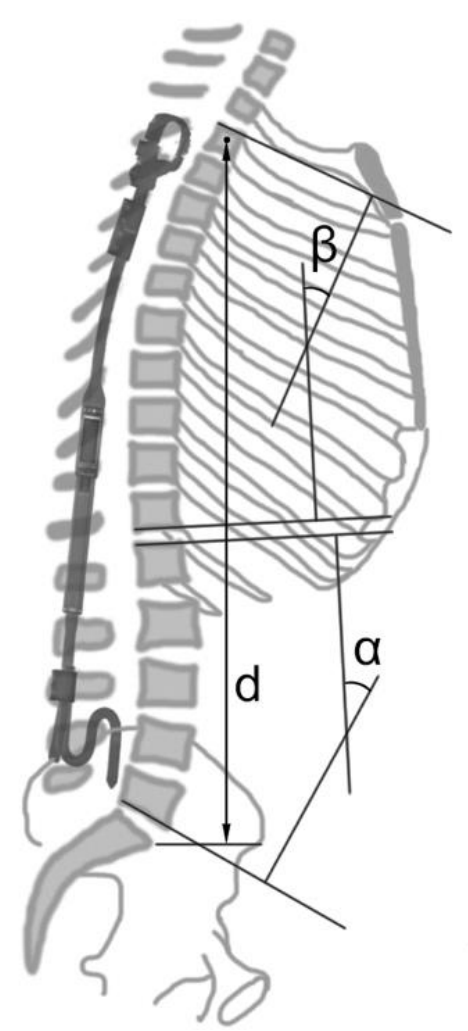

Abbildung 9: Schemazeichnung zur Messung im sagittalen Profil. Dargestellt ist eine schematische Zeichnung von lateral der Wirbelsäule mit Rippen, Beckenknochen und einem MAGEC@-Stab. Der Winkel $\alpha$ kennzeichnet die LWS-Lordose, der Winkel $\beta$ die BWS-Kyphose. Die Strecke $d$ entspricht der Wirbelsäulenlänge. (Skizze erstellt von Dr. H. Lorenz, Arbeitsgruppe Kinderorthopädie, UMG, 2015; ebenfalls verwendet in der Dissertation von Katharina Grönefeld). 


\subsubsection{Body-Mass-Index (BMI) und Gewichtsklassen}

Die Kinder wurden sowohl im Rahmen der Primärimplantation als auch der Verlängerungstermine gemessen und gewogen, damit ein Body-Mass-Index (BMI) errechnet werden konnte. Der BMI ist ein Maß für den Körperfettanteil (Kromeyer-Hauschild et al. 2001), der mit folgender Formel errechnet wird (Herold 2013):

- $\quad B M I=\mathrm{kg} / \mathrm{m}^{2}$

Ein elektronischer BMI-Rechner der Universitätsklinik Tübingen half, den BMI zu errechnen und direkt in Perzentilen für Kinder und Jugendliche einzuteilen. Hierfür nutzt die Homepage als Referenz eine Studie von Kromeyer-Hauschild et al. (2001), anhand derer es möglich wurde, die Kinder entsprechend ihres BMI in Gewichtsklassen zu unterteilen. Die Arbeitsgemeinschaft „Adipositas im Kindes- und Jugendalter“ (AGA) empfiehlt folgende Einteilung:

- Ausgeprägtes Untergewicht: $<3$. Perzentile

- Untergewicht: $<10$. Perzentile

- Normgewicht: 10.-90. Perzentile

- Übergewicht: $>$ 90. Perzentile

- Adipositas: 97.- 99,5. Perzentile

- Extreme Adipositas: > 99,5. Perzentile

\subsection{Erfassung der Komplikationen}

Die folgenden Komplikationen wurden im Rahmen dieser Doktorarbeit erfasst:

1) Perioperative Komplikationen: Pneumothorax, Embolie, Wundheilungsstörungen, Frühinfekt, Druckulzera, postoperative Schmerzen länger als drei Monate

2) Implantat-assoziierte Komplikationen: Spätinfekt (nach drei Monaten), Implantatversagen bzw. Implantatbruch, Dislokation, Rippenfraktur, Nichtdistrahierbarkeit der extern zu steuernden Implantate

3) Studienabbruch: durch persönliche oder medizinische Gründe 


\subsection{Statistische Auswertung}

Für die statistische Auswertung wurden die Programme Excel und STATISTICA (Firma StatSoft ${ }^{\circledR}$ Europe, deutsche Geschäftsführung in Hamburg) genutzt. Mittels STATISTICA wurden die Messwerte anhand der einfaktoriellen Varianzanalyse bzw. einfaktoriellen ANOVA, analysis of variance, getestet.

Das Signifikanzniveau wurde auf $\mathrm{p}<0,05\left(^{*}\right), \mathrm{p}<0,01\left(^{* *}\right)$ und $\mathrm{p}<0,001(* * *)$ festgelegt. 


\section{Ergebnisse}

\subsection{Patientenkollektiv}

\subsubsection{Alter und biologisches Geschlecht}

Das Patientenkollektiv umfasste 21 Kinder mit SMA und Wirbelsäulendeformität, worunter sich elf Jungen und zehn Mädchen fanden.

Von diesen 21 Kindern erhielten 19\% (4 Kinder) erst in der Folge, also nach vorangegangener VEPTR ${ }^{\circledR}-$ Versorgung, ein kombiniertes VEPTR ${ }^{\circledR} /$ MAGEC $®$-Implantat. Diese vier Kinder waren bei ihrer VEPTR-Implantation im Durchschnitt 6,9 Jahre alt (5,0 bis 9,2 Jahre) und bekamen gemittelt 3,5 Verlängerungen (2-5) in durchschnittlich 23,4 Monaten (17,5-31,6). Diese Gruppe wurde im durchschnittlichen Alter von 9,5 Jahren (7,8-11,5) mit dem

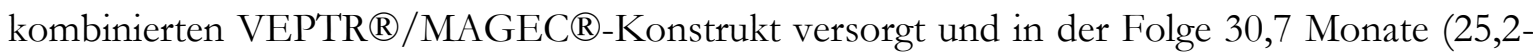
40,6) bzw. im Rahmen von 9,5 Verlängerungen (8-11) weiter begleitet. Als exemplarisches radiologisches Korrelat der Ergebnisse dient die Abbildung 10 auf Seite 27.

Die 17 Kinder, die im Rahmen der Primärimplantation mittels des MAGEC®-Implantats versorgt wurden, waren zu diesem Zeitpunkt durchschnittlich 7,5 Jahre alt (5,0-9,4). Ihr followup umfasste gemittelt 7,3 Verlängerungen (5-9) in einem Zeitraum von durchschnittlich 23,4 Monaten (17,0-33,7).

Zusammengenommen ergab sich hieraus also ein gesamtes durchschnittliches follow-up von 24,8 Monaten (17,0-40,6), in dem klinische und radiologische Daten der mit dem MAGEC®Implantat versorgten Kinder $(\mathrm{n}=21)$ erfasst wurde. 

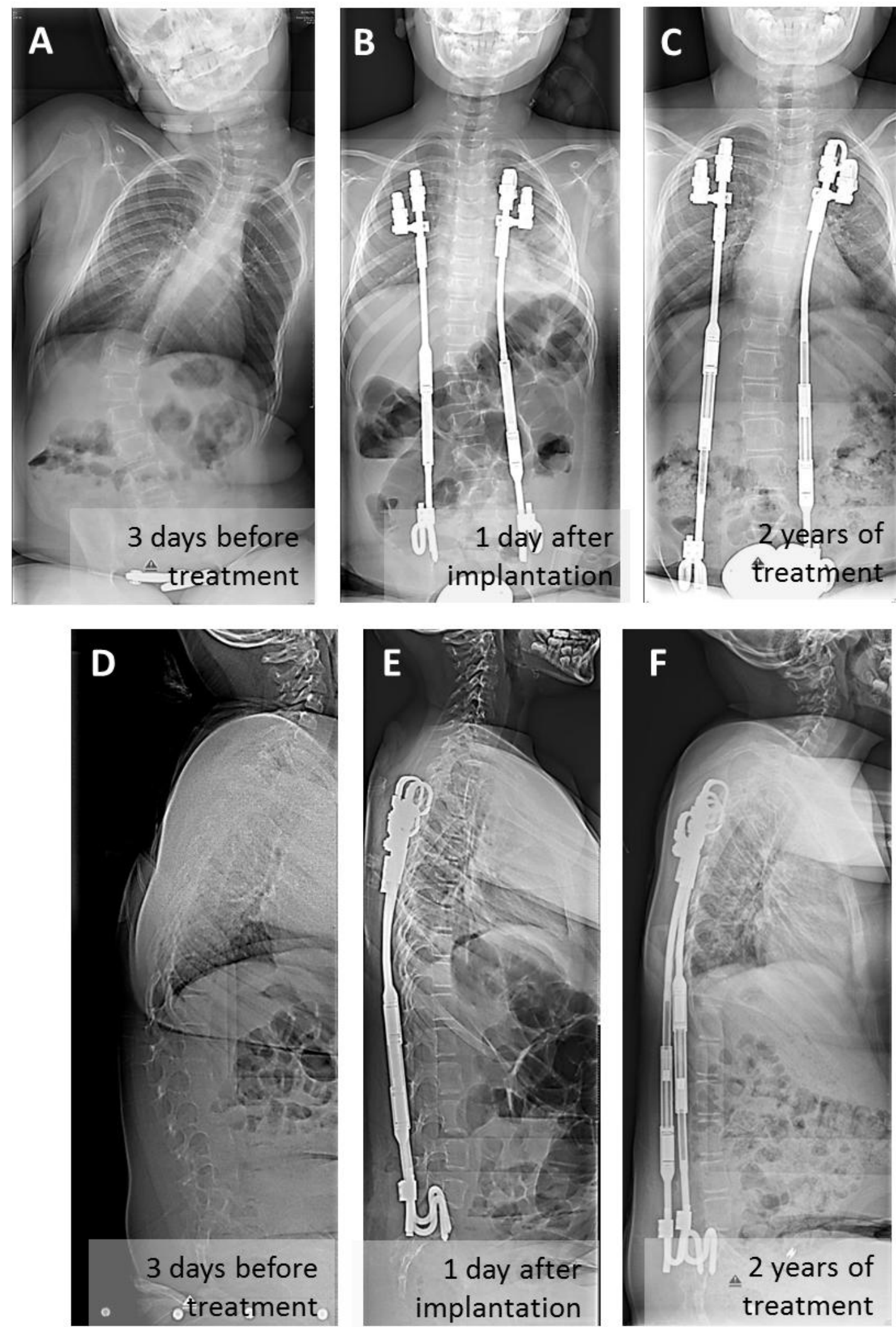

Abbildung 10: MAGEC $($-Behandlung bei SMA. A. p.- (A-C), bzw. laterale (D-F) Röntgenaufnahmen eines sieben Jahre alten Mädchens mit Skoliose und SMA. Die Hauptkrümmung von $58^{\circ}$ (A) konnte postoperativ auf $12^{\circ}$ (B) korrigiert werden. Die Korrektur konnte im 2-Jahres-Verlauf aufrechterhalten werden (C). Im sagittalen Profil (D) konnten die Kyphose und Lordose initial korrigiert werden (E), im Verlauf nahm die Kyphose wieder zu (F). Quelle: Lorenz et al. (2017) Magnetically controlled devices parallel to the spine in children with spinal muscular atrophy. JBJS Open Access 2 , e0036 
Tabelle 1: Klinische Daten des Patientenkollektivs.

\begin{tabular}{|c|c|}
\hline Variable & Anzahl \\
\hline Patienten & 21 \\
\hline weiblich & 10 \\
\hline männlich & 11 \\
\hline \multicolumn{2}{|l|}{ Primärimplantation (Patientenanzahl) } \\
\hline VEPTR $®$ & 4 \\
\hline MAGEC® & 17 \\
\hline Durchschnittsalter bei VEPTR ${ }^{\circledR}$-Implantation & $6,9(5,0-9,2)$ \\
\hline Durchschnittsalter bei MAGEC®-Implantation & $7,8(5,0-11,5)$ \\
\hline \multicolumn{2}{|l|}{ VEPTR $®$ Behandlung } \\
\hline Verlängerungen & $3,5(2-5)$ \\
\hline Verlängerungen in Monaten & $30,3(26,4-38,4)$ \\
\hline \multicolumn{2}{|l|}{ MAGEC® Behandlung } \\
\hline Verlängerungen & $8,2(5-9)$ \\
\hline Verlängerungen in Monaten & $26,7(17,3-33,3)$ \\
\hline Komplikationen (Rate in Prozent) & 21 von $325(6,5 \%)$ \\
\hline Pneumothorax; & 4 (jeweils $n=1$ ) \\
\hline \multicolumn{2}{|c|}{ OP bei Pneumothorax, Rippenfraktur und Stabdislokation; } \\
\hline \multicolumn{2}{|l|}{ Wundinfektion; } \\
\hline \multicolumn{2}{|l|}{ Postoperative Schmerzen $>3$ Monate } \\
\hline Implantat-assoziiert & 17 von 304 \\
\hline Druckulkus; & 4 (jeweils $n=1$ ) \\
\hline \multicolumn{2}{|l|}{ Implantatwanderung mit Rippenfraktur (mit und } \\
\hline \multicolumn{2}{|l|}{ ohne Stabdislokation); } \\
\hline \multicolumn{2}{|l|}{ Diskonnektion des Querauslegers } \\
\hline Nicht regelrecht ausgefahren & 13 \\
\hline
\end{tabular}




\subsubsection{Grunderkrankung}

Das Studienkollektiv umfasste ausschließlich Kinder mit spinaler Muskelatrophie und Wirbelsäulendeformität. Das Krankheitsbild ist unter „1.2 Spinale Muskelatrophie“ beschrieben.

\subsubsection{Datenmaterial}

Es wurden insgesamt 704 Röntgenbilder im Rahmen von 192 radiologischen Untersuchungen - zur Verbesserung der Messgenauigkeit - von jeweils zwei Untersuchern vermessen. Hiervon entstanden im Rahmen des VEPTR ${ }^{\circledR}$-Einbaus und der operativen VEPTR ${ }^{\circledR}$-Verlängerungen bei vier Kindern in 18 Untersuchungen 72 Röntgenbilder. In der Gruppe von vier Kindern,

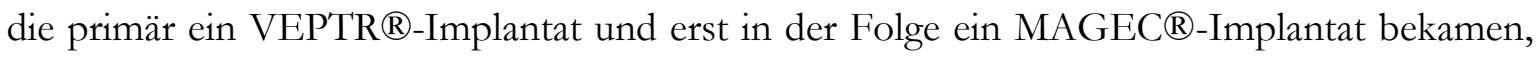
wurden im Rahmen des MAGEC®-Einbaus und der nicht-invasiven MAGEC®Verlängerungen 42 radiologische Untersuchungen mit 161 Röntgenbildern vorgenommen. Im Rahmen dieser Untersuchungen konnten aus technischen Gründen folgende Röntgenbilder nicht ausgewertet werden:

- zwei a. p.-Röntgenbilder nach der Verlängerung

- zwei lat.-Röntgenbilder vor der Verlängerung

- zwei lat.-Röntgenbilder nach der Verlängerung

In der Gruppe der 17 Kinder, welche die Primärimplantation mittels eines MAGEC®Systems erfuhren, entstanden im Rahmen von 132 Messungen 471 Röntgenbilder. Hier konnten aus technischen Gründen folgende Röntgenbilder nicht ausgewertet werden:

- $\quad$ ein a. p.-Röntgenbild nach der Verlängerung

- drei lat.-Röntgenbilder nach der Verlängerung

Es konnten also von den 704 Röntgenbildern insgesamt 1,4\% der Bilder nicht in der Statistik berücksichtigt werden.

Da ab Juni 2015 (29.06.2015) aus medizinischen Gründen nur noch Röntgenbilder nach der Distraktion angefertigt wurden, gab es im Rahmen von 28 Verlängerungsterminen lediglich 
die Röntgenbilder, die nach den Verlängerungen gemacht wurden. Dies betraf in einem Fall eine Untersuchung eines Kindes, das in der Gruppe war, die primär mit einem VEPTR ${ }^{\circledR}$ Implantat versorgt wurde und in 27 Fällen Untersuchungen von sieben Kindern, die im Rahmen der Primärimplantation das MAGEC®-Implantat bekamen.

Weil die Kinder zu unterschiedlichen Zeitpunkten operiert wurden, resultierten daraus unterschiedlich viele follow-up-Daten. So ergab sich, dass einige Kinder von Beginn ihrer MAGEC®-Implantation bis zum Zeitpunkt der Versteifung ihrer Wirbelsäule, der Spondylodese, begleitet wurden und ihre Messungen in der Statistik auftauchen und dass es wiederum bei anderen Kindern lediglich fünf Verlängerungstermine gab, deren Daten in der Statistik Verwendung fanden.

\subsection{Ausmaß der Skoliose in der Koronarebene}

\subsubsection{Skoliosekrümmungswinkel (Cobb-Winkel) des Gesamtkollektivs im Verlauf}

Der Skoliosekrümmungswinkel diente in dieser Studie zur quantitativen Erfassung der Ausprägung der kindlichen Skoliose in der a. p.-Ebene.

Die Abbildung 11 auf S. 32 zeigt den durchschnittlichen Skoliosekrümmungswinkel aufgetragen gegen die Zeit. Unterteilt wurde das Patientenkollektiv in drei Gruppen: Die erste Gruppe bestand aus $\mathrm{n}=4$ Kindern (blaue Raute), bei welchen primär ein VEPTR ${ }^{\circledR}-$ System implantiert wurde. Dargestellt ist mit den blauen Rauten der Zeitraum zwischen der VEPTR ${ }^{\circledR}$-Primärimplantation und dem Austausch gegen einen MAGEC®-Implantat. Die zweite Teilgruppe (rote Quadrate) umfasste alle Kinder, die keine spinale Voroperation erfuhren, also nicht zunächst mittels eines VEPTR ${ }^{\circledR}$-Implantats versorgt wurden. Dargestellt sind mit den roten Quadraten die Messwerte vor und nach der MAGEC ${ }^{-}$Primärimplantation. Die dritte Gruppe (grüne Dreiecke) fasst ab der ersten Verlängerungsprozedur die erstgenannten Gruppen zusammen und zeigt das Verhalten des Skoliosekrümmungswinkels bis zum Ende des Beobachtungszeitraumes. In Klammern findet sich unter den jeweiligen Verlängerungen eine Angabe über die Größe des Patientenkollektivs, das in die Messung mit einbezogen wurde. Diese Zahlen variieren, da einige Winkel nicht reproduziert werden konnten (aufgrund von fehlenden Bildern oder schlechter Bildqualität).

Die voroperierte Gruppe (blaue Raute) mit Implantat-Wechsel wies nach dem MAGEC®Einbau eine deutliche Reduktion des Skoliosekrümmungswinkels von 41,2\% (30,8 $\left.\mathrm{zu} 18,1^{\circ}\right)$ auf, welche statistisch allerdings nicht signifikant war. Ein hoch signifikantes Ergebnis ergab sich jedoch in der zweiten, nicht voroperierten Gruppe (rotes Quadrat) direkt postoperativ 
nach MAGEC®-Einbau. Sie verzeichneten eine Verringerung des Skoliosekrümmungswinkels um $57,5 \%$ von präoperativ $69,6^{\circ}$ zu postoperativ $29,6^{\circ} \quad(p<0,001)$. Aus den Rohdaten erschloss sich in der zusammengefassten Gruppe $(n=21)$ eine Verringerung des Skoliosekrümmungswinkels um $55,9^{\%}$ von $62,2^{\circ}$ präoperativ zu $27,4^{\circ}$ postoperativ.

In der Folge wurde beobachtet, wie sich der Skoliosekrümmungswinkel auch im Verlauf entwickelte (Abbildung 11, grüne Dreiecke, $n=21$ ). Hier wurden beide Gruppen gemeinsam betrachtet und jeweils der gemittelte Winkel vor und nach den Verlängerungsprozeduren aufgetragen. Die Korrektur der Skoliose konnte im Verlauf aufrechterhalten werden. So betrug der Skoliosekrümmungswinkel nach der sechsten Verlängerung, die zuvor als Endpunkt definierte wurde, 33,8 $(\mathrm{n}=19)$. Das entspricht einer Zunahme der Skoliose um durchschnittlich $6,4^{\circ} \mathrm{im}$ Vergleich zu den unmittelbar postoperativ ermittelten Werten.

Je mehr Verlängerungen mit einbezogen wurden, desto kleiner wurde die Studiengruppe (n). So finden sich bei der letzten Verlängerung nur noch sechs Kinder. Zur Anschauung wurden diese Daten mit einbezogen (s. Abbildung 11). 


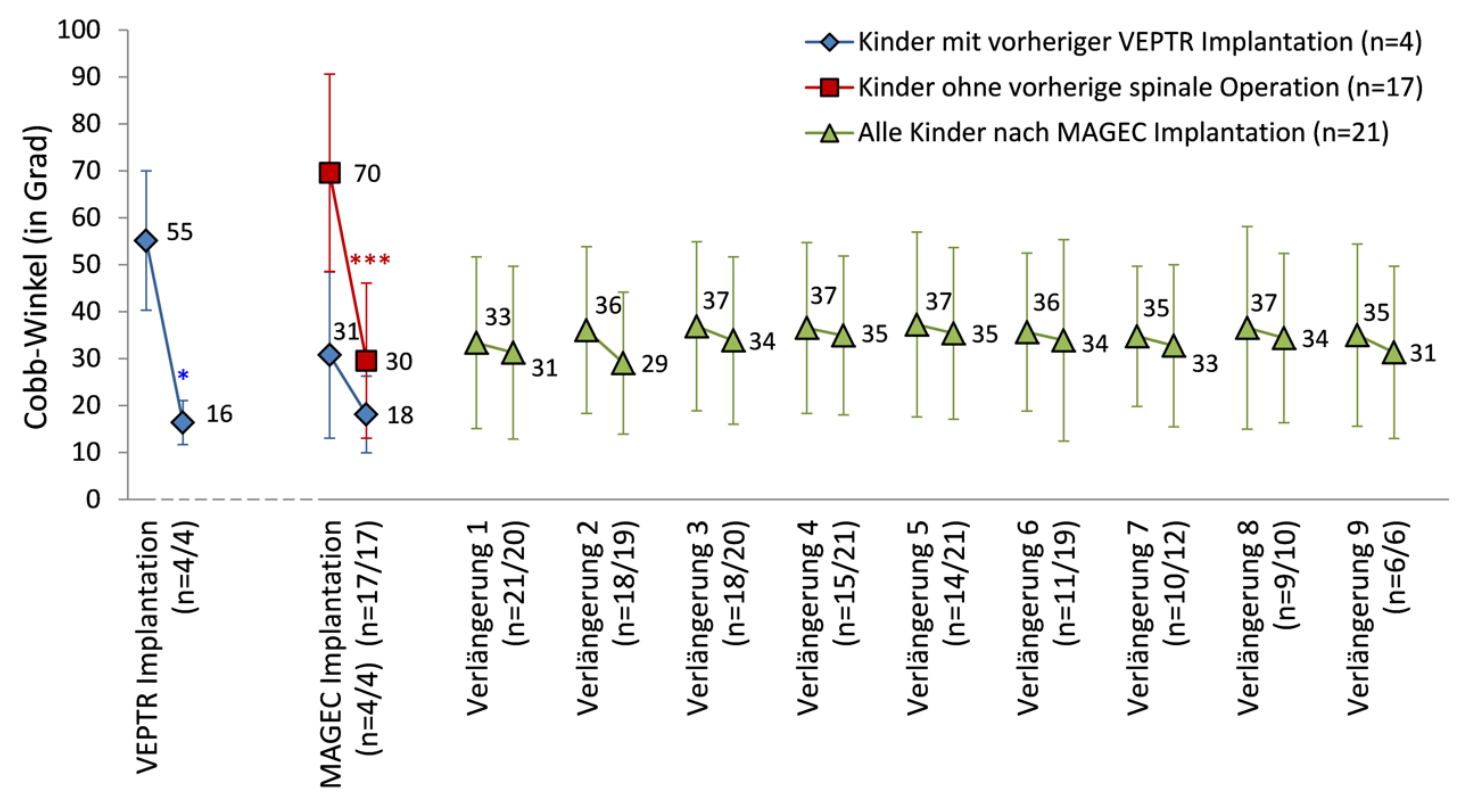

Zeitpunkte (prä/post Intervention)

Abbildung 11: Skoliosekrümmungswinkel (Cobb-Winkel) des Gesamtkollektivs ( $\mathrm{n}=21)$. Dargestellt ist der Skoliosekrümmungswinkel während des gesamten Untersuchungszeitraums. Die farbliche Kennzeichnung markiert die drei Untersuchungsgruppen. Die blaue Gruppe umfasst die Messungen der VEPTR ${ }^{\circledR}$-Behandlung; prä- und postoperativ sowie bei Wechsel des Implantats mit MAGEC ${ }^{-}$Einbau. In der roten Gruppe wurden die Messwerte vor und nach MAGEC®-Implantation der Kinder zusammengefasst, die ausschließlich mit einem MAGEC®-Implantat behandelt wurden. Beide Subgruppen wurden nach der MAGEC®-Implantation während der ambulanten externen Verlängerungen zur grünen Gruppe zusammengefasst. Zu jedem Messwert ist die Standardabweichung und die Anzahl der ausgewerteten Patienten ( $\mathrm{n}=$ vor Verlängerung/nach Verlängerung) angegeben. Die Zeit vom MAGEC®-Einbau bis zur ersten Verlängerung umfasste 5 Monate, zwischen den jeweiligen nachfolgenden Verlängerungen lagen 3 Monate. ${ }^{*} \mathrm{p}<0,05 ;{ }^{* * *} \mathrm{p}<0,001$

\subsubsection{Skoliosekrümmungswinkel in Abhängigkeit vom Alter}

Betrachtet man anhand der Abbildung 12 die Studiengruppe in zwei Altersgruppen geteilt, nämlich jeweils unter sechs Jahren ( $\mathrm{n}=4$; blaue Raute) und im Alter von sechs bis zehn Jahren ( $\mathrm{n}=15$; rotes Quadrat), so wird ersichtlich, dass insgesamt in beiden Gruppen signifikante Ergebnisse erzielt wurden. Die Gruppe der unter Sechsjährigen wies jedoch initial eine stärkere Abnahme des Skoliosekrümmungswinkels von $66,5 \%$ auf $\left(61,5^{\circ} \mathrm{zu} 20,6^{\circ}\right)$. Die

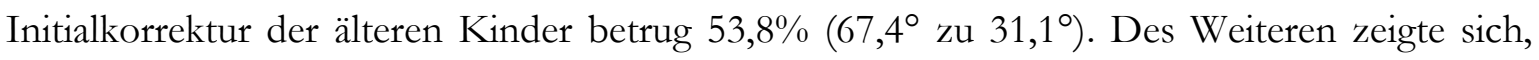
dass beide Gruppen nach der sechsten Verlängerung nach wie vor eine sehr ( $<6$ Jahren) bzw. hoch (6-10 Jahre) signifikante Abnahme des Skoliosekrümmungswinkels aufwiesen. Hierbei ist 
zu vermerken, dass die Gruppe der sechs- bis zehnjährigen Kinder bei jeder Verlängerung bis zum Endpunkt V6 ein hoch signifikantes Ergebnis erzielen konnte, die Gruppe der unter Sechsjährigen einmalig (V1) ein hoch signifikantes Ergebnis zeigte und ansonsten sehr signifikante Ergebnisse aufwies.

Die Daten zweier Kinder fanden aufgrund ihres Alters (über zehn Jahre) keine Verwendung in dieser Grafik.

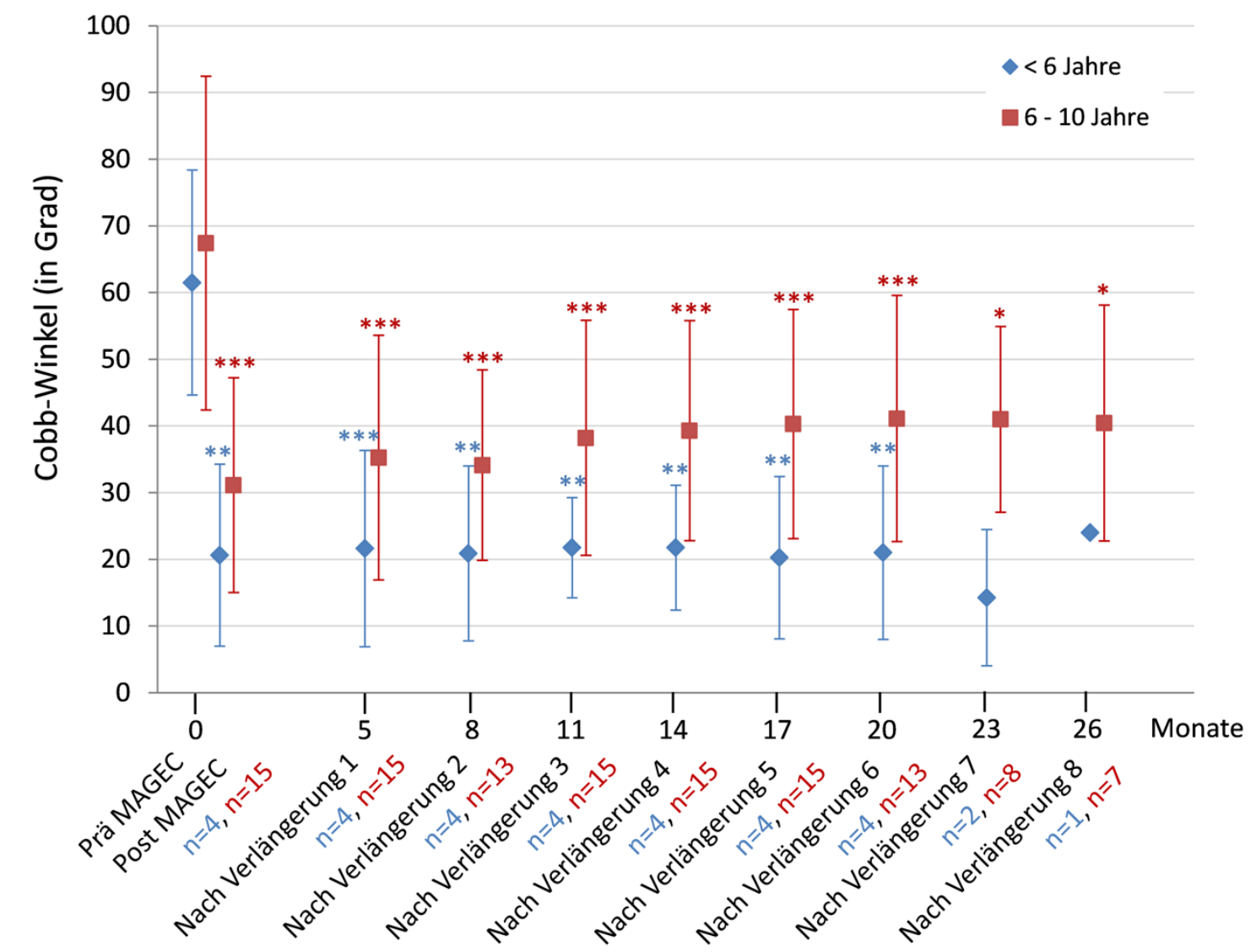

Abbildung 12: Skoliosekrümmungswinkel gruppiert nach Alter. Dargestellt ist der Skoliosekrümmungswinkel vor und nach dem MAGEC®-Einbau und nach jeder Verlängerung bis zur 8. Verlängerung, gruppiert nach Alter bei MAGEC®-Einbau. Die blaue Gruppe zeigt die Messwerte der <6-jährigen Kinder, die rote Gruppe der 6-10-jährigen Kinder. Zu jedem Messwert ist die Standardabweichung und die Anzahl der ausgewerteten Patienten (n) angegeben. Die Zeit vom MAGEC®-Einbau bis zur ersten Verlängerung umfasste 5 Monate, zwischen den jeweiligen nachfolgenden Verlängerungen lagen 3 Monate. ${ }^{*} \mathrm{p}<0,05 ;{ }^{* *} \mathrm{p}<0,01 ; * * * \mathrm{p}<0,001$

\subsubsection{Skoliosekrümmungswinkel in Abhängigkeit vom Kinder-BMI}

In Abbildung 13 wurden die Kinder in Gruppen entsprechend ihres BMI eingeteilt; Übergewicht (grünes Dreieck, $n=8$ ), Normalgewicht (rotes Quadrat, $n=4$ ) und Untergewicht (blaue Raute, $\mathrm{n}=8$ ). Die Grafik zeigt keine deutlichen Unterschiede zwischen den Subgruppen. 
Es konnte jedoch gezeigt werden, dass alle Kinder direkt postoperativ eine signifikante Verminderung ihrer Skoliose erfuhren, diese aber unterschiedlich stark ausfiel. Waren die Kinder über- oder untergewichtig, erfuhr ihre Skoliose direkt postoperativ eine hoch signifikante Verminderung $(p<0,001)$, waren sie normalgewichtig wurde eine sehr signifikante Verringerung erzielt $(p<0,01)$. Die Gruppe der untergewichtigen Kinder zeigte auch bei den Verlängerungsprozeduren zweimalig hoch signifikante Ergebnisse (V1 und V3). Es wurde ersichtlich, dass alle Kinder, unabhängig ihres BMI, beim Endpunkt V6 weiterhin eine sehr signifikante Verminderung des Skoliosekrümmungswinkels in Bezug zum präoperativen Ausgangswert aufwiesen.

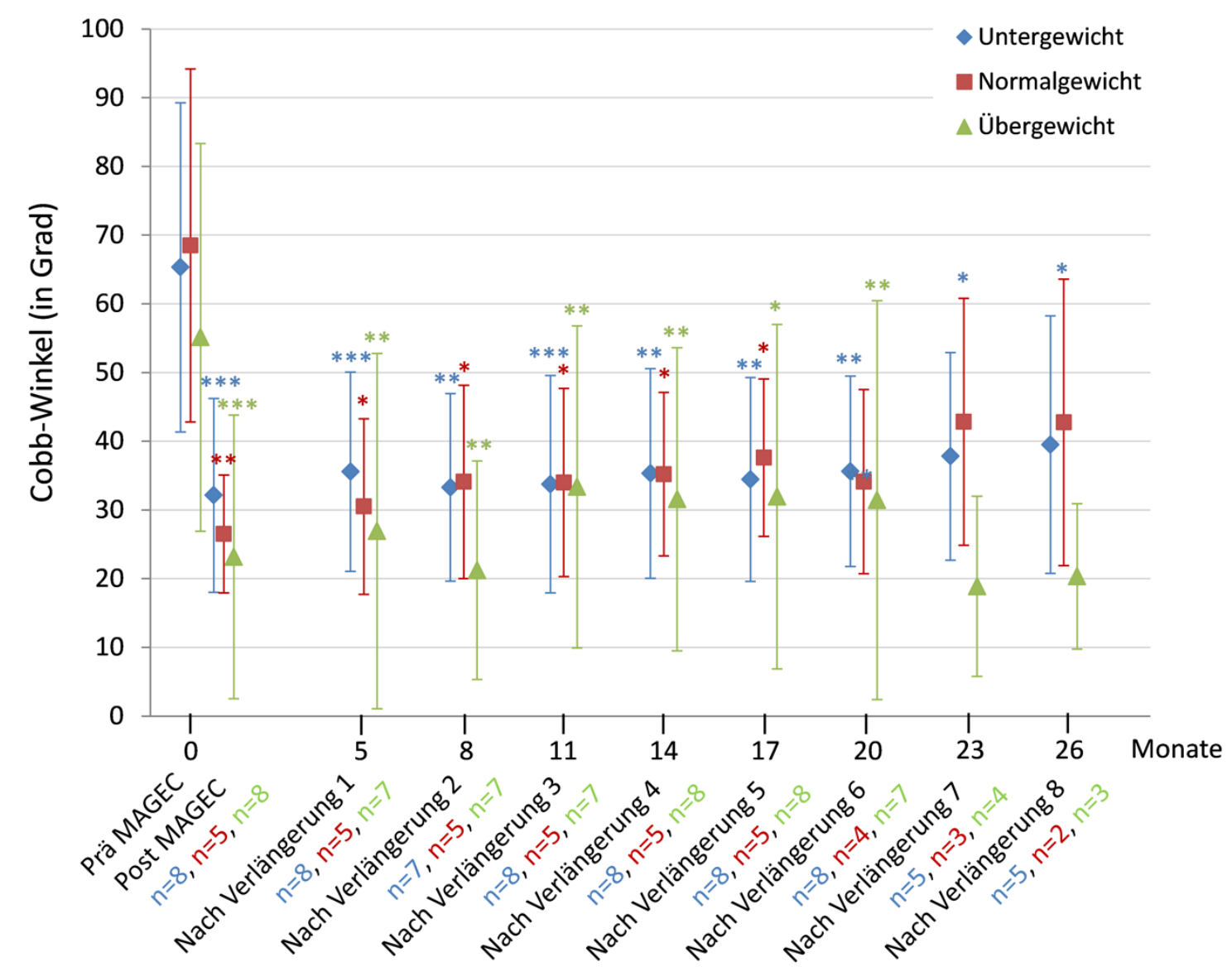

Abbildung 13: Skoliosekrümmungswinkel gruppiert nach BMI. Dargestellt ist der Skoliosekrümmungswinkel vor und nach dem MAGEC®-Einbau und nach jeder Verlängerung bis zur 8. Verlängerung, gruppiert nach Kinder-BMI bei MAGEC ${ }^{\circledR}$-Einbau (Untergewicht=blaue Gruppe; Normalgewicht $=$ rote Gruppe; Übergewicht=grüne Gruppe). $\mathrm{Zu}$ jedem Messwert sind die Standardabweichung und die Anzahl der ausgewerteten Patienten (n) angegeben. Die Zeit vom MAGEC®-Einbau bis zur ersten Verlängerung umfasste 5 Monate, zwischen den jeweiligen nachfolgenden Verlängerungen lagen 3 Monate. ${ }^{*} \mathrm{p}<0,05 ;{ }^{* *} \mathrm{p}<0,01 ;{ }^{* *} \mathrm{p}<0,001$ 


\subsection{Beckenschiefstand (pelvic obliquity)}

\subsubsection{Beckenschiefstand des Gesamtkollektivs im Verlauf}

In Abbildung 14 ist das Ausmaß des Beckenschiefstandes gegen die Zeit dargestellt. Die Patientenkollektiveinteilung erfolgte analog der Darstellung für den Skoliosekrümmungswinkel.

Es konnte gezeigt werden, dass ein vorhandener Beckenschiefstand in beiden Gruppen (rot und blau) jeweils durch die bilaterale VEPTR®- oder MAGEC®-Implantation signifikant verbessert werden konnte, wobei die nicht voroperierte Gruppe $(n=17)$ ein hoch signifikantes Ergebnis mit einer Verminderung von 75\% aufwies. Im Behandlungsverlauf (grüne Dreiecke) konnten die niedrigen postoperativen Werte gehalten werden. 


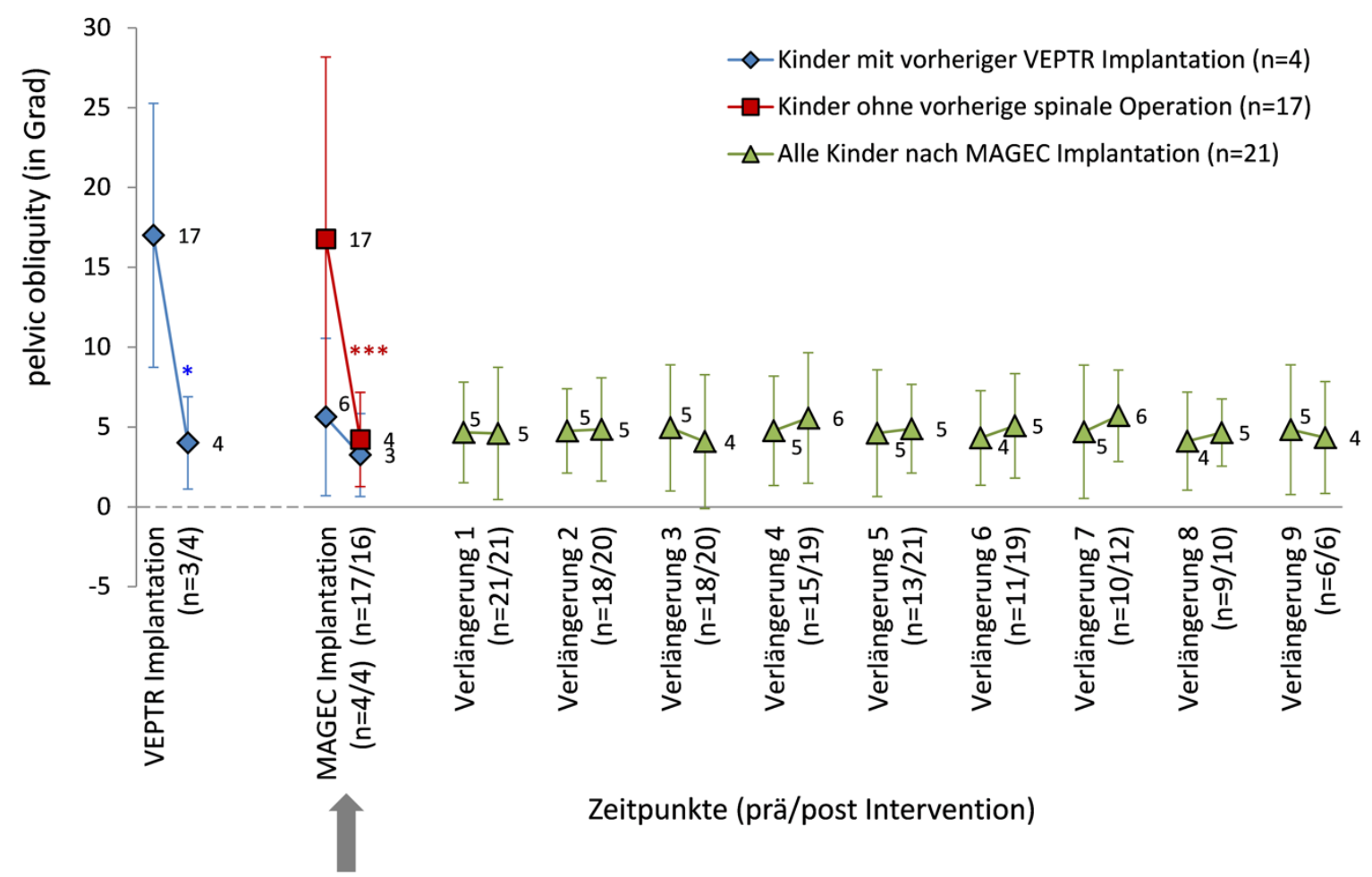

Abbildung 14: Beckenschiefstand (pelvic obliquity) des Gesamtkollektivs ( $\mathrm{n}=21$ ). Dargestellt ist der Beckenschiefstand während des gesamten Untersuchungszeitraums. Die farbliche Kennzeichnung markiert die drei Untersuchungsgruppen. Die blaue Gruppe umfasst die Messungen der VEPTR ${ }^{\circledR}$ Behandlung; prä- und postoperativ sowie bei Wechsel des Implantats mit MAGEC®-Einbau. In der roten Gruppe wurden die Messwerte vor und nach MAGEC®-Implantation der Kinder zusammengefasst, die ausschließlich mit einem MAGEC®-Implantat behandelt wurden. Beide Subgruppen wurden nach der MAGEC®-Implantation während der ambulanten Verlängerungen zur grünen Gruppe zusammengefasst. Zu jedem Messwert sind die Standardabweichung und die Anzahl der ausgewerteten Patienten $\left(\mathrm{n}=\right.$ vor V/nach V) angegeben. Die Zeit vom MAGEC ${ }^{\circledR}$-Einbau bis zur ersten Verlängerung umfasste 5 Monate, zwischen den jeweiligen nachfolgenden Verlängerungen lagen 3 Monate. ${ }^{*} \mathrm{p}<0,05 ; * * * \mathrm{p}<, 001$

\subsubsection{Beckenschiefstand in Abhängigkeit vom Alter}

Teilt man die Studiengruppe wiederum in zwei Altersgruppen, nämlich jeweils unter sechs Jahre (blaue Raute) oder im Alter von sechs bis zehn Jahren (rotes Quadrat) und trägt die Winkel prä- und postoperativ und bis zur achten Verlängerung auf, so ergibt sich die Abbildung 15. Die Grafik zeigt keine signifikanten Unterschiede zwischen den Subgruppen. Statistisch konnte in der roten Gruppe über die Zeit eine signifikante Verringerung gemessen werden, dies gelang bei der blauen Gruppe aufgrund der kleineren Fallzahl nicht. 


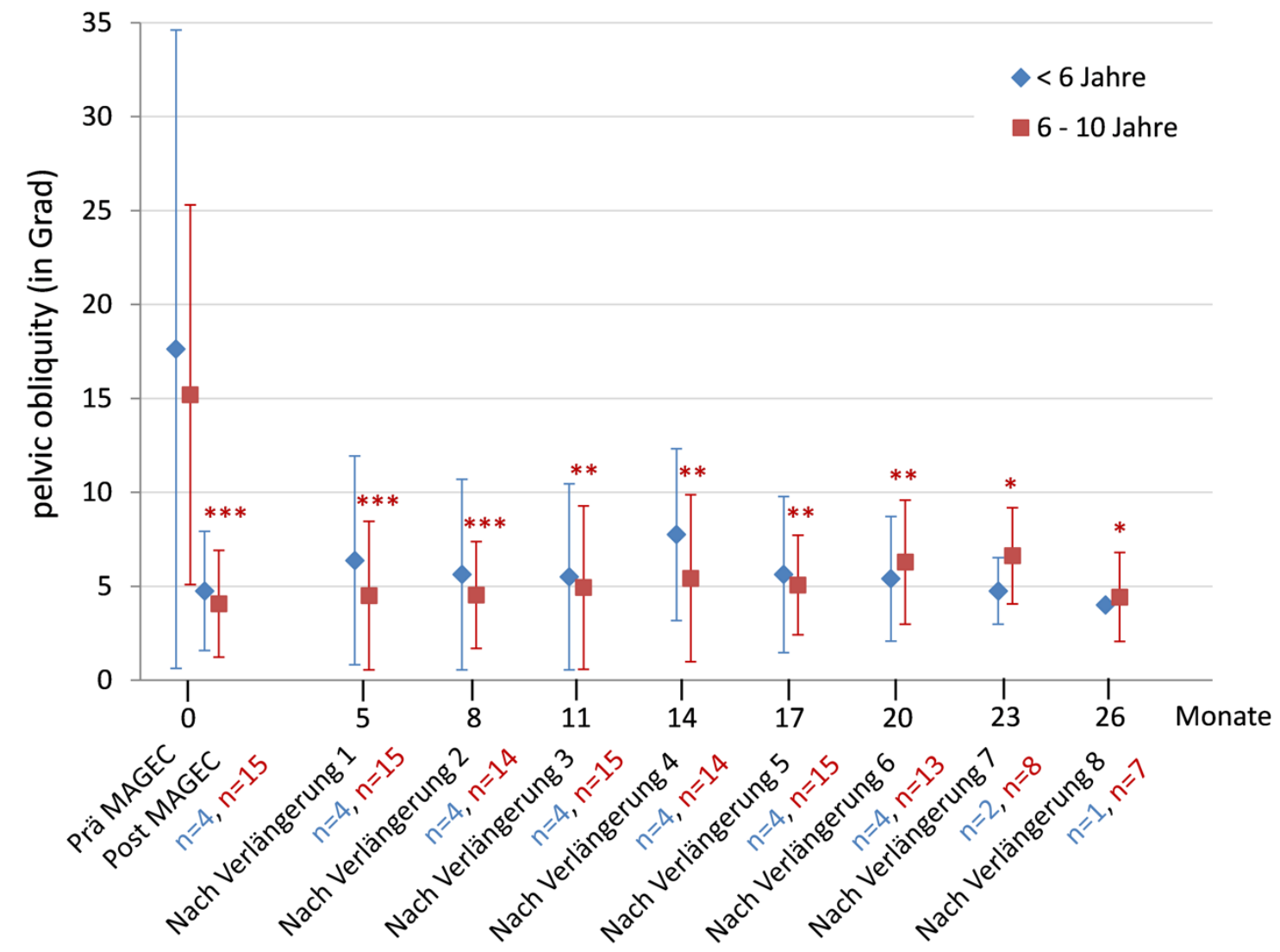

Abbildung 15: Beckenschiefstand gruppiert nach Alter. Dargestellt ist die pelvic obliquity vor und nach dem MAGEC®-Einbau und nach jeder Verlängerung bis zur 8. Verlängerung, gruppiert nach Alter bei MAGEC®-Einbau. Die blaue Gruppe zeigt die Messwerte der <6-jährigen Kinder, die rote Gruppe der 6-10-jährigen Kinder. Zu jedem Messwert sind die Standardabweichung und die Anzahl der ausgewerteten Patienten (n) angegeben. Die Zeit vom MAGEC®-Einbau bis zur ersten Verlängerung umfasste 5 Monate, zwischen den jeweiligen nachfolgenden Verlängerungen lagen 3 Monate. ${ }^{*} \mathrm{p}<0,05$; ${ }^{* *} \mathrm{p}<0,01 ; * * * \mathrm{p}<0,001$

\subsubsection{Beckenschiefstand in Abhängigkeit vom Kinder-BMI}

In Abbildung 16 sind die Mittelwerte des Beckenschiefstandes vor und nach MAGEC®Implantation und nach jeder Verlängerung bis zur 8. Verlängerung, gruppiert entsprechend dem BMI - Übergewicht (grünes Dreieck, $n=8$ ), Normalgewicht (rotes Quadrat, $n=4$ ) und Untergewicht (blaue Raute, $\mathrm{n}=8$ ) - dargestellt.

Auch diese Grafik zum BMI zeigt keine deutlichen Unterschiede zwischen den Subgruppen. Es konnten lediglich in der Gruppe der normalgewichtigen Kinder (rot) und zweimalig in der Gruppe der Übergewichtigen (grün) signifikante Ergebnisse erzielt werden. So konnte die signifikante Verminderung des Beckenschiefstandes in der normalgewichtigen Gruppe (rotes Quadrat) direkt postoperativ von $17,7^{\circ}$ auf $3,2^{\circ}$ bis zur fünften Verlängerung mit $5,3^{\circ}$ aufrechterhalten werden. 
Der Beckenschiefstand der unter- als auch der übergewichtigen Kinder, mit Ausnahme der Messwerte der oben genannten Verlängerungen, wies sowohl nach der Operation als auch nach den einzelnen Verlängerungen keine signifikante Verminderung auf.

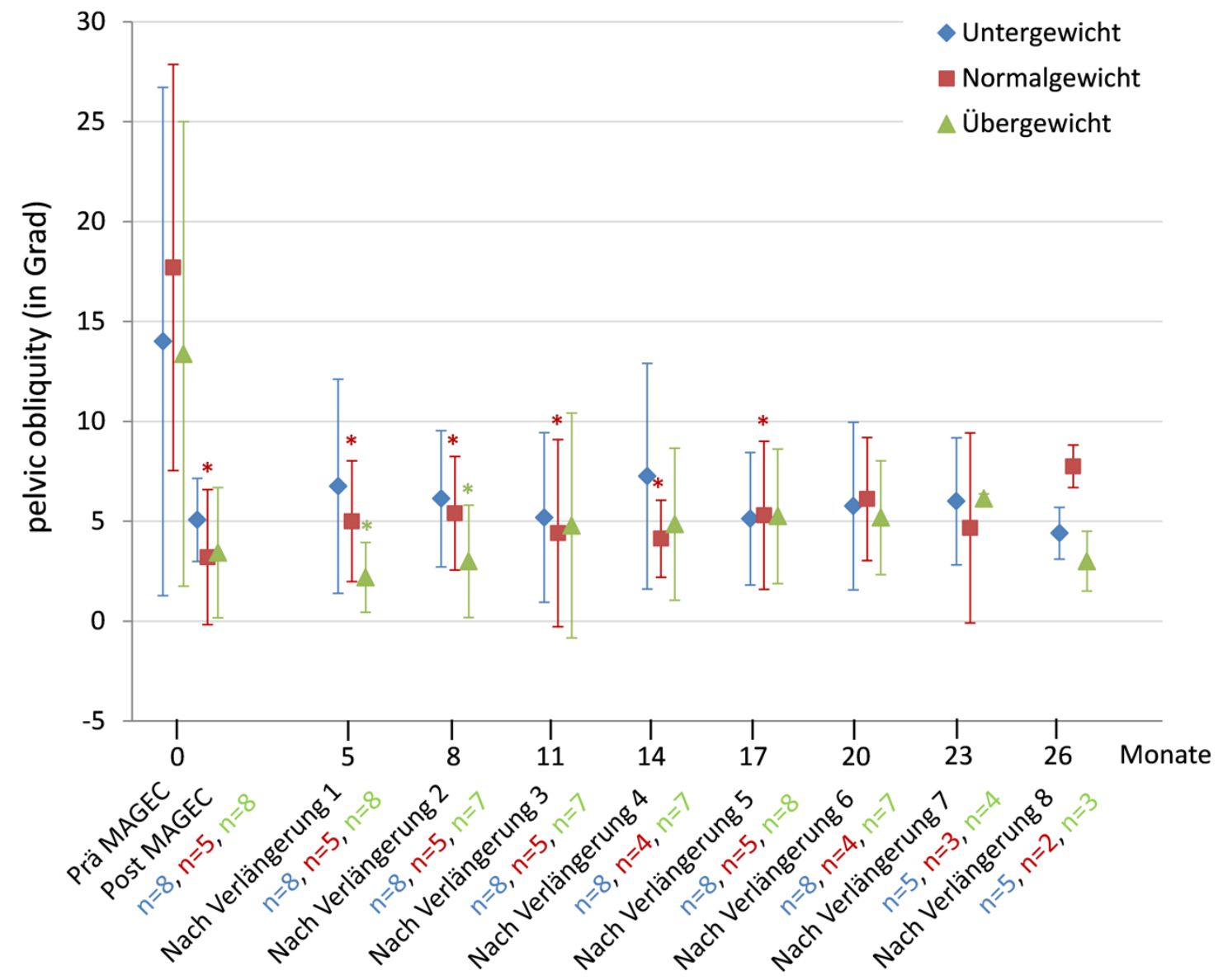

Abbildung 16: Beckenschiefstand gruppiert nach BMI. Dargestellt ist der Beckenschiefstand vor und nach dem MAGEC®-Einbau und nach jeder Verlängerung bis zur 8. Verlängerung, gruppiert nach Kinder-BMI bei MAGEC ${ }^{\circledR}$-Einbau (Untergewicht=blaue Gruppe; Normalgewicht=rote Gruppe; Übergewicht=grüne Gruppe). Zu jedem Messwert sind die Standardabweichung und die Anzahl der ausgewerteten Patienten (n) angegeben. Die Zeit vom MAGEC®-Einbau bis zur ersten Verlängerung umfasste 5 Monate, zwischen den jeweiligen nachfolgenden Verlängerungen lagen 3 Monate. ${ }^{*} \mathrm{p}<0,05$

\subsection{Thorakale Kyphose}

\subsubsection{Thorakale Kyphose des Gesamtkollektivs im Verlauf}

In Abbildung 17 ist die thorakale altersadaptierte Kyphose von der VEPTR®-Implantation, über den MAGEC®-Einbau bis hin zur 9. Verlängerung dargestellt. In dieser Grafik finden sich analog der vorherigen Auswertungen erneut drei Teilgruppen wieder. 
Da die Angabe der Kyphose nur als Abweichung zur per Formel errechneten altersabhängigen Norm sinnhaft ist (,altersadaptierte Kyphose“, s. „2.4.2.4 Thorakale Kyphose und lumbale Lordose"), wurde in dieser Grafik der Wert 0 mit der altersabhängigen Norm gleichgesetzt. So sind positive Werte in der Grafik eine Abweichung in Richtung Hyperkyphose, während negative Werte eine Abweichung in Richtung Hypokyphose bedeuten.

Aus den Rohdaten zur Abbildung 17 kann gelesen werden, dass sich die Kyphose der Kinder mit VEPTR ${ }^{\circledR}$-Implantation ( $\mathrm{n}=4$, blaue Raute) von einer Hyperkyphose von 9,50 präoperativ $\mathrm{zu} 8,5^{\circ}$ postoperativ änderte und in der Folge durch die MAGEC®-Implantation eine Annäherung bis auf $0,5^{\circ}$ an die Norm erfuhren. Die initiale MAGEC $®$-Implantation bei den nicht voroperierten Kindern ( $\mathrm{n}=17$, rotes Quadrat) sowie die folgenden Verlängerungen (grünes Dreieck) zeigten, dass die größte Korrektur direkt postoperativ auf $7,2^{\circ}$ erfolgte. Ebenso wird ersichtlich, dass es in der Folge wiederum eine Tendenz Richtung Hyperkyphose zum ursprünglichen Ausgangswert gab, bei zu berücksichtigendem abnehmendem Patientenkollektiv mit steigender Verlängerungszahl. Eine Hypokyphose, also ein Flachrücken, wurde durch die Implantate nicht hervorgerufen. 


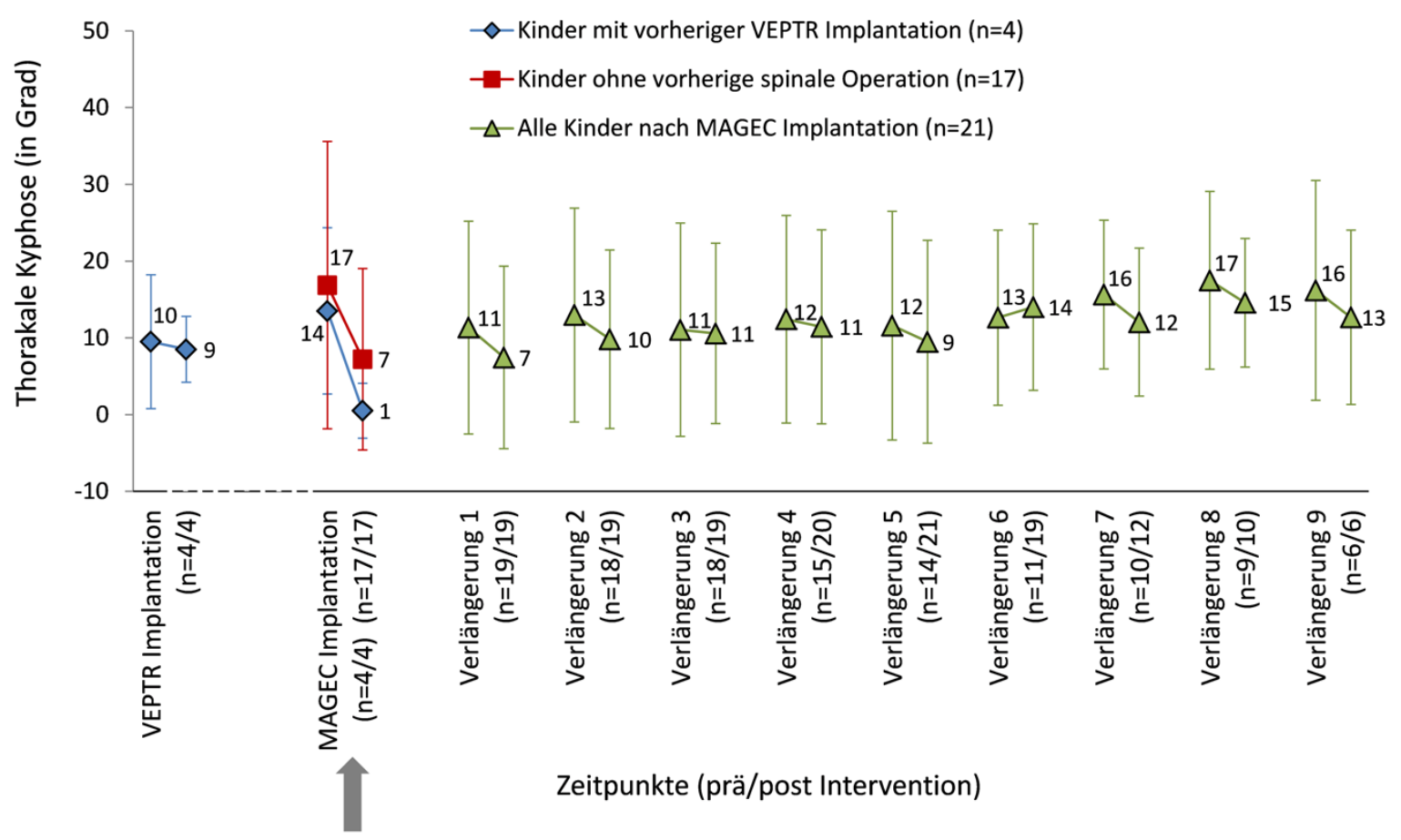

Abbildung 17: Thorakale Kyphose des Gesamtkollektivs ( $\mathrm{n}=21$ ). Dargestellt ist die altersadaptierte thorakale Kyphose während des gesamten Untersuchungszeitraums. Die farbliche Kennzeichnung markiert die drei Untersuchungsgruppen. Die blaue Gruppe umfasst die Messungen der VEPTR®Behandlung; prä- und postoperativ sowie bei Wechsel des Implantats mit MAGEC®-Einbau. In der roten Gruppe wurden die Messwerte vor und nach MAGEC®-Implantation der Kinder zusammengefasst, die ausschließlich mit einem MAGEC®-Implantat behandelt wurden. Beide Subgruppen wurden nach der MAGEC®-Implantation während der ambulanten Verlängerungen zur grünen Gruppe zusammengefasst. Zu jedem Messwert sind die Standardabweichung und die Anzahl der ausgewerteten Patienten ( $\mathrm{n}=$ vor V/nach V) angegeben. Die Zeit vom MAGEC@-Einbau bis zur ersten Verlängerung umfasste 5 Monate, zwischen den jeweiligen nachfolgenden Verlängerungen lagen 3 Monate.

\subsubsection{Thorakale Kyphose in Abhängigkeit vom Alter}

Abbildung 18 zeigt die thorakale altersadaptierte Kyphose präoperativ, postoperativ und jeweils nach jeder Verlängerungsprozedur bis zur achten Verlängerung, unterteilt in zwei Gruppen, unter 6 Jahre (blaue Raute, $\mathrm{n}=4$ ) und von sechs bis zehn Jahren (rotes Quadrat, $\mathrm{n}=15)$.

Auffallend war eine Abnahme um 74,8\% von präoperativ $27,0^{\circ}$ auf postoperativ $6,8^{\circ}$ in der Gruppe der unter 6-jährigen Kinder, was ein sehr signifikantes Ergebnis darstellte $(p<0,01)$. Der Winkel nahm in den folgenden Verlängerungen zwar wieder zu, doch konnte bei der letzten Verlängerung 6 ein nach wie vor signifikantes Ergebnis in Bezug zum Ausgangswert ermittelt werden (Annäherung auf 9,0 an die altersabhängige Norm). 
In der Gruppe der 6- bis 10-Jährigen konnte weder postoperativ noch nach den Verlängerungsprozeduren ein signifikantes Ergebnis verzeichnet werden.

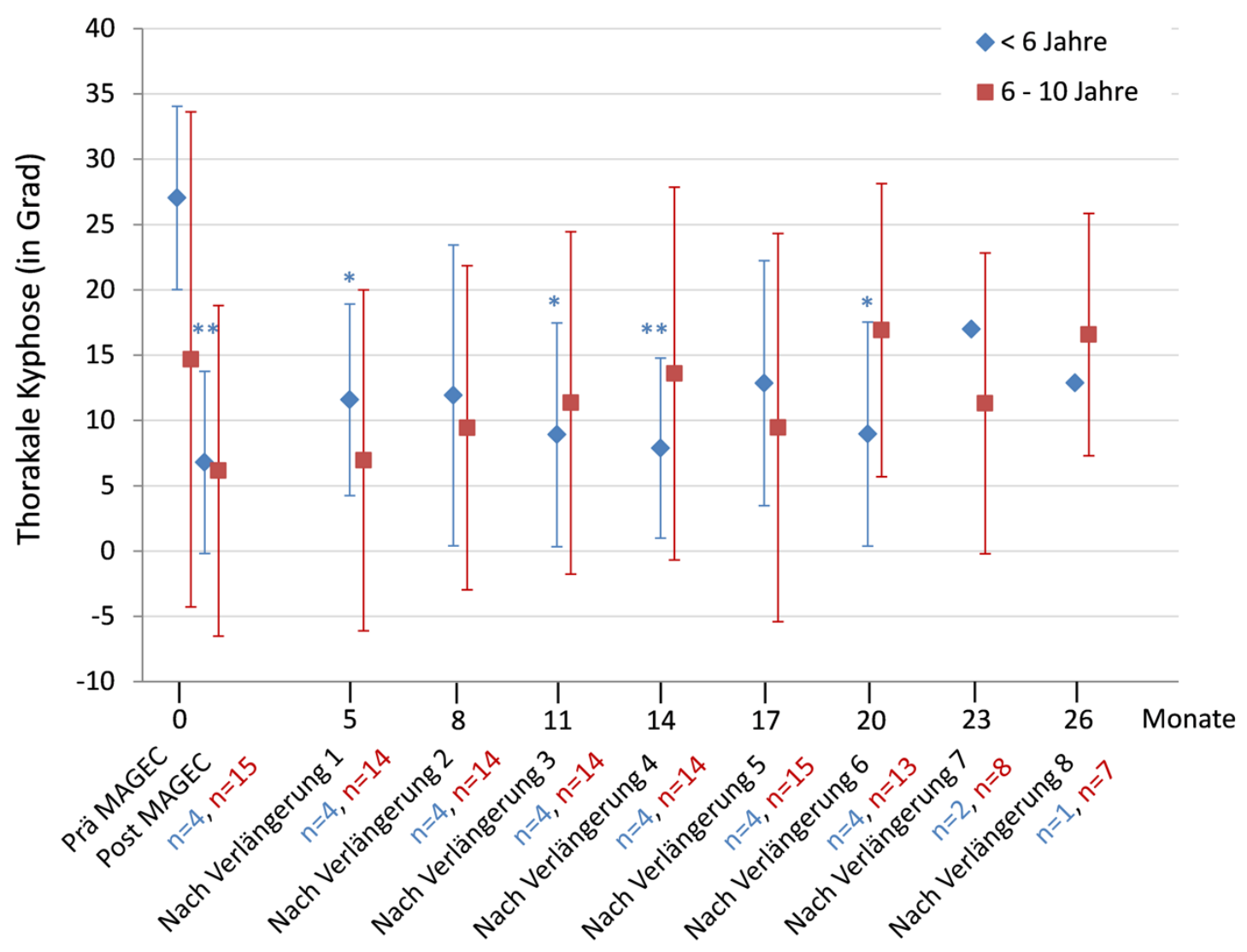

Abbildung 18: Thorakale Kyphose gruppiert nach Alter. Dargestellt ist die altersadaptierte thorakale Kyphose vor und nach dem MAGEC®-Einbau und nach jeder Verlängerung bis zur 8. Verlängerung, gruppiert nach Alter bei MAGEC®-Einbau. Die blaue Gruppe zeigt die Messwerte der <6-jährigen Kinder, die rote Gruppe der 6-10-jährigen Kinder. Zu jedem Messwert sind die Standardabweichung und die Anzahl der ausgewerteten Patienten (n) angegeben. Die Zeit vom MAGEC®-Einbau bis zur ersten Verlängerung umfasste 5 Monate, zwischen den jeweiligen nachfolgenden Verlängerungen lagen 3 Monate. ${ }^{*} \mathrm{p}<0,05 ;{ }^{*} \mathrm{p}<0,01$

\subsubsection{Thorakale Kyphose in Abhängigkeit vom Kinder-BMI}

In Abbildung 19 ist die altersadaptierte thorakalen Kyphose unter MAGEC®-Behandlung in Abhängigkeit vom BMI bis zum 8. Verlängerungstermin dargestellt. Es wurden drei Teilgruppen gebildet, eine übergewichtige (blaue Raute, $n=8$ ), eine normalgewichtige (rotes Quadrat, $\mathrm{n}=5$ ) und eine untergewichtige (grünes Dreieck, $\mathrm{n}=8$ ).

Insgesamt zeigt die Grafik, dass die Kyphose in allen Subgruppen relativ konstant und ohne signifikante Änderungen bleibt. 
Zweimalig wurden signifikante Ergebnisse erzielt, und zwar eine Verringerung der Hyperkyphose von $17,9^{\circ}$ präoperativ in der Gruppe der übergewichtigen Kinder auf $5,8^{\circ}$ nach der vierten und auf $4,9^{\circ}$ nach der fünften Verlängerungsprozedur. Insgesamt hatte das Gewicht bei dieser Untersuchung keinen Einfluss auf die altersadaptierte Kyphose.

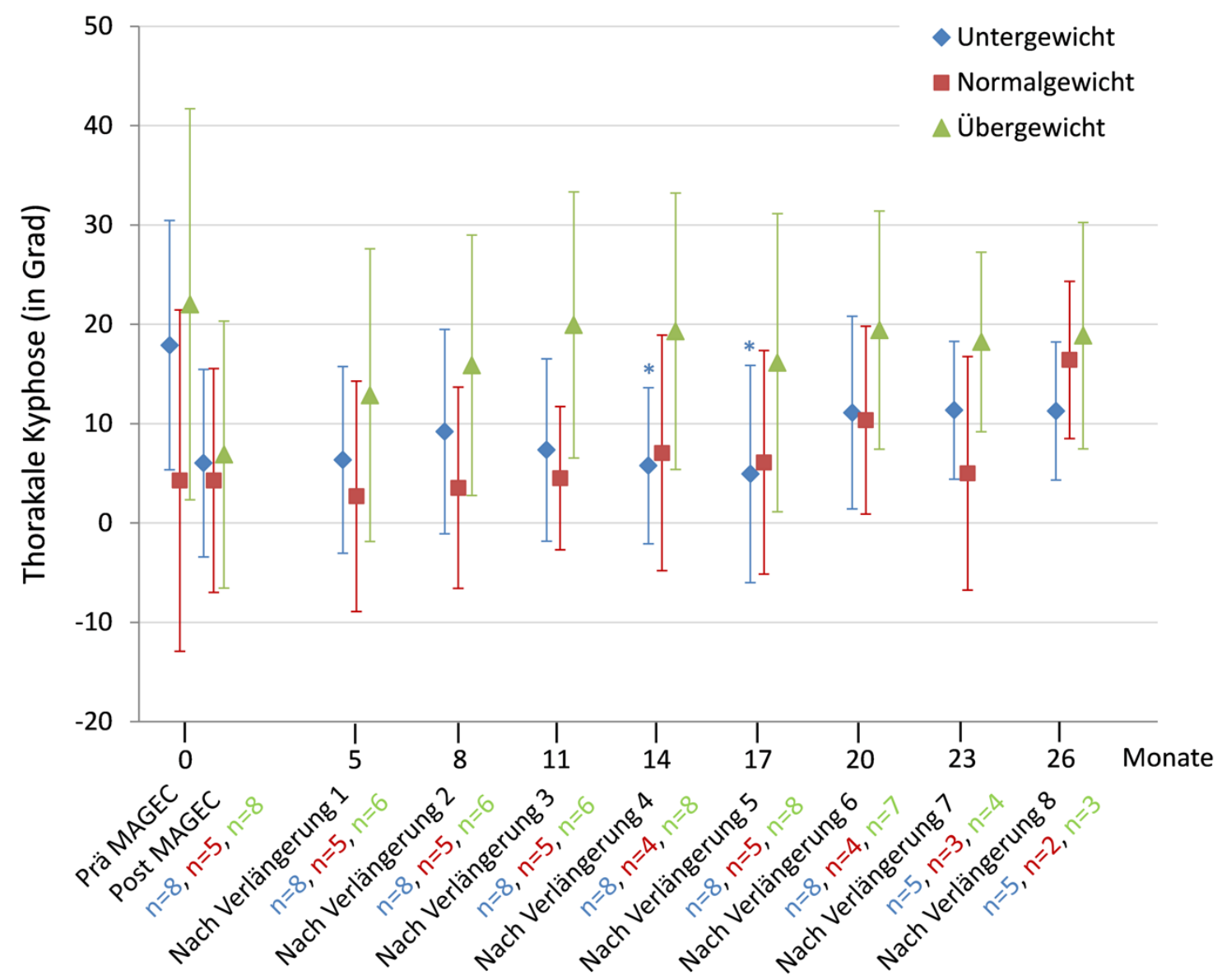

Abbildung 19: Thorakale Kyphose gruppiert nach BMI. Dargestellt ist die altersadaptierte thorakale Kyphose vor und nach dem MAGEC®-Einbau und nach jeder Verlängerung bis zur 8. Verlängerung, gruppiert nach Kinder-BMI bei MAGEC®-Einbau (Untergewicht=blaue Gruppe; Normalgewicht=rote Gruppe; Übergewicht=grüne Gruppe). $\mathrm{Zu}$ jedem Messwert sind die Standardabweichung und die Anzahl der ausgewerteten Patienten (n) angegeben. Die Zeit vom MAGEC®-Einbau bis zur ersten Verlängerung umfasste 5 Monate, zwischen den jeweiligen nachfolgenden Verlängerungen lagen 3 Monate. ${ }^{*} \mathrm{p}<0,05$ 


\subsection{Lumbale Lordose}

\subsubsection{Lumbale Lordose des Gesamtkollektivs im Verlauf}

In Abbildung 20 ist die altersadaptierte lumbale Lordose gegen die Zeit dargestellt. In dieser Grafik finden sich wie zuvor auch drei Teilgruppen wieder, welche analog gekennzeichnet sind.

Für die altersadaptierte Lordose gilt bezüglich der grafischen Darstellung dasselbe wie für die Kyphose (s. „2.4.2.4 Thorakale Kyphose und lumbale Lordose“): Eine Angabe des Winkels ist auch hier lediglich sinnvoll im Kontext zur altersabhängigen Norm, die sich hier im Wert 0 wiederfindet. Positive Werte entsprechen also einer Hyperlordose, negative Werte einer lumbalen Abflachung bzw. relativen Lumbalkyphose.

In der Gruppe mit vorheriger VEPTR ${ }^{\circledR}$-Implantation (blaue Raute, $\mathrm{n}=4$ ) ist durch die primäre Operation keine Veränderung der Lordose zu verzeichnen. Die MAGEC®_ Operation führte jedoch zu einer Verminderung von durchschnittlich $6,8^{\circ}$, wodurch in dieser Teilgruppe im Vergleich zur altersabhängigen Norm eine Hypolordose entstand $\left(-0,6^{\circ}\right)$. Die Gruppe, die als Primärimplantation das MAGEC®-System erhielt (rotes Quadrat, n=17), verzeichnete eine Zunahme der altersadaptierten Lordose von $2,2^{\circ}$ auf $3,3^{\circ}$ postoperativ, wobei diese Abweichung im Messwertbereich liegt. Für die Darstellung der altersadaptierten Lordose im Rahmen der Verlängerungen wurden beide Gruppen zusammengefasst (grünes Dreieck). Hier war zu sehen, dass die Lordose bis zur siebten Verlängerung abnahm $\left(-7,7^{\circ}\right)$, bzw. einer Hypolordose entsprach und somit eine relative Kyphosierung der LWS entstand. Diese Ergebnisse sind jedoch nicht signifikant. In der Folge wiederum näherten sich die Werte der altersabhängigen Norm an $\left(\mathrm{V} 9:-1,1^{\circ}\right)$, wobei allerdings das kleiner werdende Patientenkollektiv zu beachten ist. 


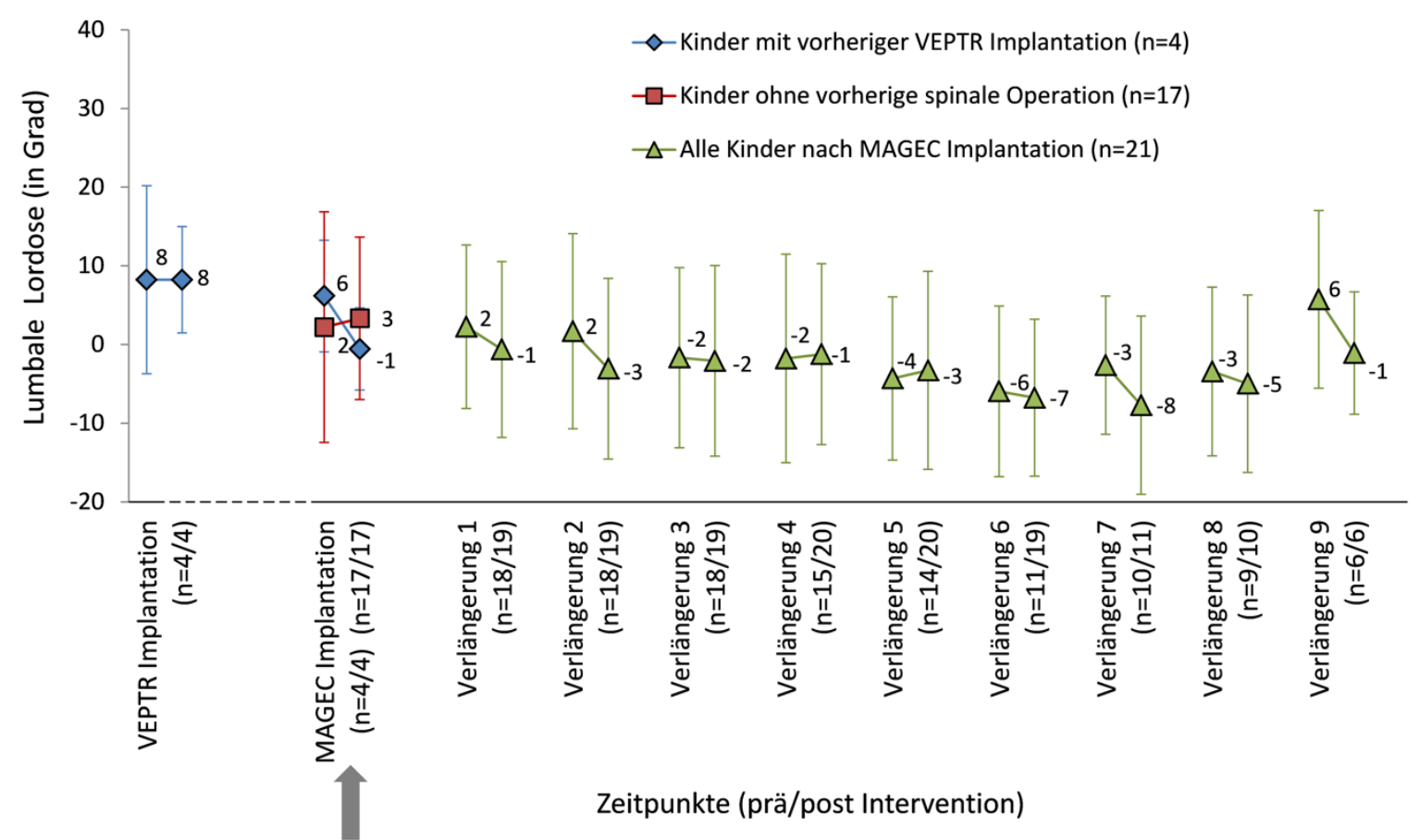

Abbildung 20: Lumbale Lordose des Gesamtkollektivs ( $n=21)$. Dargestellt ist die altersadaptierte lumbale Lordose während des gesamten Untersuchungszeitraums. Die farbliche Kennzeichnung markiert die drei Untersuchungsgruppen. Die blaue Gruppe umfasst die Messungen der VEPTR ${ }^{\circledR}$ Behandlung; prä- und postoperativ sowie bei Wechsel des Implantats mit MAGEC®-Einbau. In der roten Gruppe wurden die Messwerte vor und nach MAGEC®-Implantation der Kinder zusammengefasst, die ausschließlich mit einem MAGEC®-Implantat behandelt wurden. Beide Subgruppen wurden nach der MAGEC®-Implantation während der perkutanen Verlängerungen zur grünen Gruppe zusammengefasst. Zu jedem Messwert sind die Standardabweichung und die Anzahl der ausgewerteten Patienten ( $\mathrm{n}=$ vor V/nach V) angegeben. Die Zeit vom MAGEC®-Einbau bis zur ersten Verlängerung umfasste 5 Monate, zwischen den jeweiligen nachfolgenden Verlängerungen lagen 3 Monate.

\subsubsection{Lumbale Lordose in Abhängigkeit vom Alter}

Eine Darstellung der altersadaptierten Lordose vor und nach MAGEC®-Einbau sowie bis zur 8. Verlängerung in Abhängigkeit vom Alter findet sich in Abbildung 21 wieder. Die blaue Raute zeigt den Verlauf der Lordose bei der Gruppe der unter Sechsjährigen ( $n=4)$, die rote Kurve spiegelt den Verlauf der Sechs- bis Zehnjährigen ( $n=15)$ wider. Die Grafik zeigt keine deutlichen Unterschiede zwischen den Subgruppen. Die Lordose beider Gruppen veränderte sich von prä- zu postoperativ nicht signifikant. In der Folge nahm der Winkel in beiden 
Gruppen negative Werte an, so betrug er in der Gruppe mit den jüngeren Kindern am Endpunkt V6 $-5,1^{\circ}$ und in der Gruppe der älteren Kinder -7,3 .

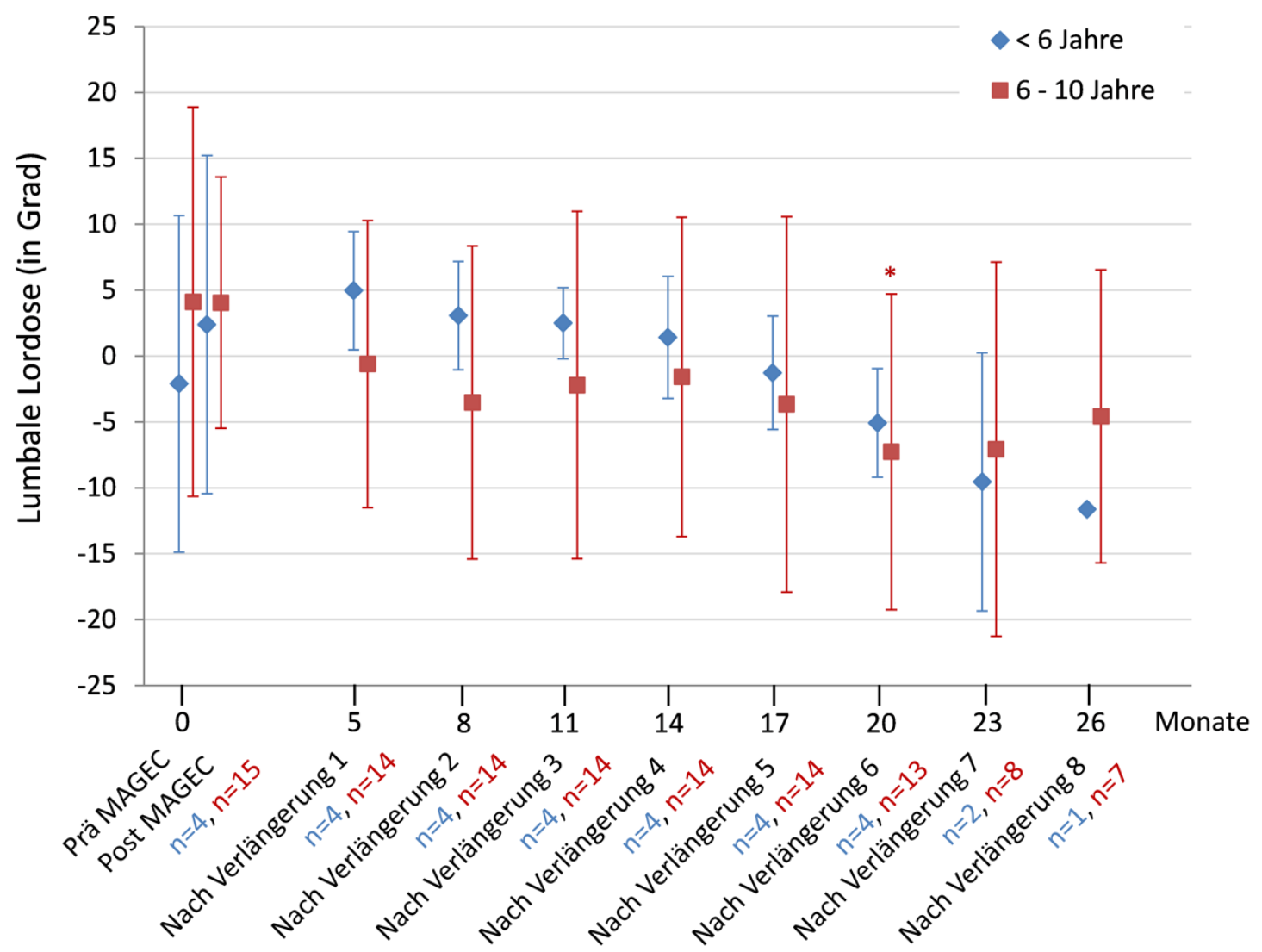

Abbildung 21: Lumbale Lordose gruppiert nach Alter. Dargestellt ist die altersadaptierte lumbale Lordose vor und nach dem MAGEC®-Einbau und nach jeder Verlängerung bis zur 8. Verlängerung, gruppiert nach Alter bei MAGEC®-Einbau. Die blaue Gruppe zeigt die Messwerte der <6-jährigen Kinder, die rote Gruppe der 6-10-jährigen Kinder. Zu jedem Messwert sind die Standardabweichung und die Anzahl der ausgewerteten Patienten (n) angegeben. Die Zeit vom MAGEC®-Einbau bis zur ersten Verlängerung umfasste 5 Monate, zwischen den jeweiligen nachfolgenden Verlängerungen lagen 3 Monate. ${ }^{*} \mathrm{p}<0,05$

\subsubsection{Lumbale Lordose in Abhängigkeit vom Kinder-BMI}

Die altersadaptierte Lordose im Verlauf in Abhängigkeit vom BMI ist in Abbildung 22 dargestellt. Die blaue Raute zeigt den Verlauf der übergewichtigen Kinder ( $n=8)$, das rote Quadrat den der normalgewichtigen $(n=5)$ und das grüne Dreieck den der untergewichtigen Kinder ( $\mathrm{n}=8)$. Während die normal- und untergewichtigen Kinder präoperativ im hyperlordotischen Bereich mit 7,7 (Untergewicht) und 3,3 $3^{\circ}$ (Normalgewicht) starteten, lagen die übergewichtigen Kinder zu Beginn mit $-1,9^{\circ}$ im hypolordotischen Bereich. Die übergewichtigen Kinder näherten sich postoperativ $0^{\circ}$, also der alterabhängigen Norm, an und bewegten sich bis zur 2. Verlängerung auf einem gering hypolordotischen Plateau $\left(-0,1^{\circ}\right.$ bis - 
$\left.1,2^{\circ}\right)$, wonach sie bis zur 5 . Verlängerung hyperlordotische Werte erreichten $\left(3,0^{\circ}\right)$, um nach der 6. Verlängerung wieder einen Wert $\left(-3,5^{\circ}\right)$ näher dem präoperativen zu erreichen. Die Lordose der normalgewichtigen Kinder nahm postoperativ wenig zu (von $3,2^{\circ}$ auf $3,6^{\circ}$ ) und erreichte in der Folge hypolordotische Werte, die nach der 7. Verlängerung am größten waren $\left(-13,0^{\circ}\right)$ und zweimalig signifikante Ergebnisse darstellten $(p<0,05$, V6 und V7). Die untergewichtigen Kinder starteten ebenfalls mit einer Hyperlordose $\left(7,7^{\circ}\right)$, die im Verlauf kontinuierlich abnahm (V6: $\left.-6,5^{\circ}\right)$. Im Rahmen der vierten und fünften Verlängerung konnten hier signifikante Veränderungen der Lordose vernommen werden $(p<0,05)$, die sechste und siebte Verlängerung erbrachte ebenfalls signifikante Ergebnisse $(p<0,01)$, wobei die Lordose nach der siebten Verlängerung die größte Abweichung von der altersabhängigen Norm darstellte $\left(-9,7^{\circ}\right)$.

Es zeigte sich, dass in allen Gruppen durch den MAGEC®-Einbau keine signifikante Veränderung der lumbalen Lordose erzielt wurde. Bei Betrachtung des Endpunktes V6 konnte in allen drei Gruppen eine Tendenz zum lumbalen Flachrücken gefunden werden. 


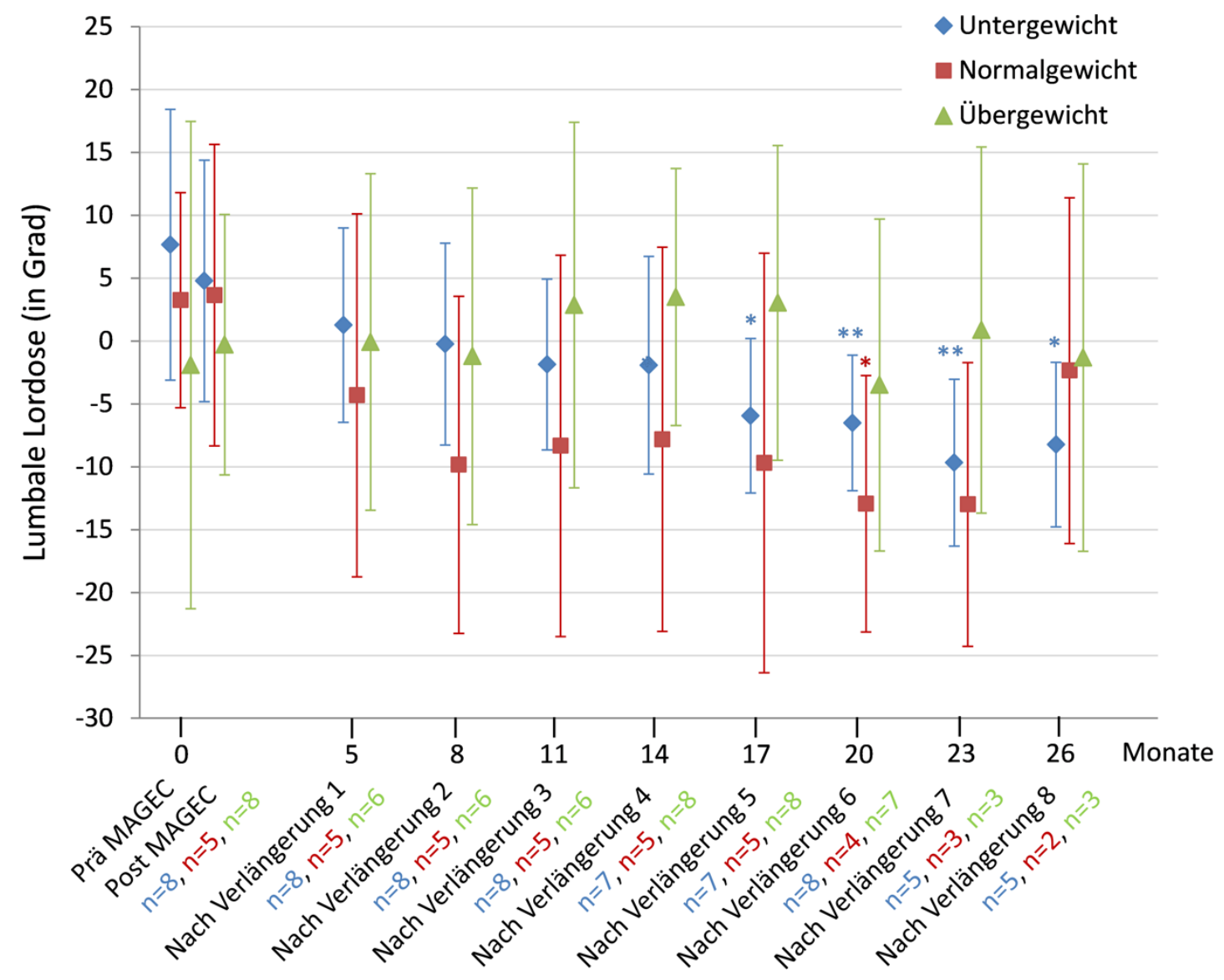

Abbildung 22: Lumbale Lordose gruppiert nach BMI. Dargestellt ist die altersadaptierte lumbale Lordose vor und nach dem MAGEC®-Einbau und nach jeder Verlängerung bis zur 8. Verlängerung, gruppiert nach Kinder-BMI bei MAGEC®-Einbau (Untergewicht=blaue Gruppe; Normalgewicht=rote Gruppe; Übergewicht=grüne Gruppe). Zu jedem Messwert sind die Standardabweichung und die Anzahl der ausgewerteten Patienten (n) angegeben. Die Zeit vom MAGEC®-Einbau bis zur ersten Verlängerung umfasste 5 Monate, zwischen den jeweiligen nachfolgenden Verlängerungen lagen 3 Monate. ${ }^{*} \mathrm{p}<0,05 ;{ }^{* *} \mathrm{p}<0,01$

\subsection{Wirbelsäulenlänge (spinal length)}

\subsubsection{Wirbelsäulenlänge des Gesamtkollektivs im Verlauf}

Die Abbildung 23 zeigt die Wirbelsäulenlänge aufgetragen gegen die Zeit. Wiederum wurde das Patientenkollektiv analog in drei Teilgruppen unterteilt.

Zunächst wird in der Grafik deutlich, dass die nicht voroperierten Kinder durch den MAGEC®-Einbau eine signifikante Zunahme der Wirbelsäulenlänge um 21,8\% bzw. $51 \mathrm{~mm}$ (von $234 \mathrm{~mm}$ zu $285 \mathrm{~mm}$, durchschnittliches Alter 7,5 Jahre) aufwiesen ( $<<0,001)$. Durch den Einbau des VEPTR ${ }^{\circledR}-K o n s t r u k t s ~(22 \mathrm{~mm})$, wie auch durch dessen Umbau in ein MAGEC®- 
System $(13 \mathrm{~mm})$ erfuhren die Kinder keinen signifikanten Zuwachs ihrer lumbalen und thorakalen Wirbelsäulenlänge (von $287 \mathrm{~mm}$ zu $300 \mathrm{~mm}$, durchschnittliches Alter 9,5 Jahre). Ferner wurde deutlich, dass im Rahmen der Verlängerungen eine nahezu lineare kontinuierliche Zunahme erfolgte, welche jedoch unter dem extern verlängerten Wert lag. Nach Primärimplantation zeigte sich vor der ersten ambulanten Verlängerung nach 5 Monaten eine Reduktion der zuvor dokumentierten Wirbelsäulenlänge.

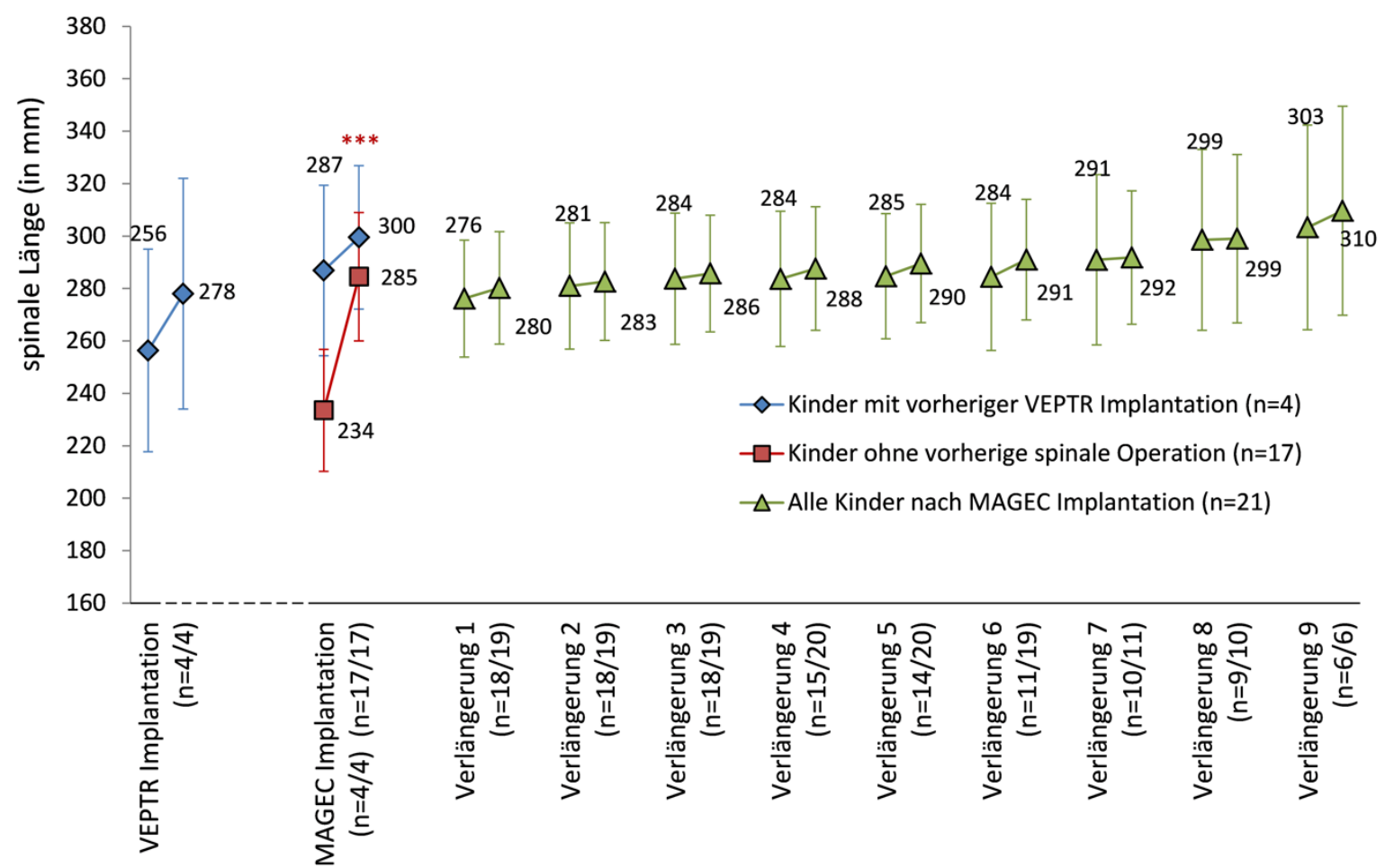

Zeitpunkte (prä/post Intervention)

Abbildung 23: Wirbelsäulenlänge (spinal length) des Gesamtkollektivs ( $\mathrm{n}=21)$. Dargestellt ist die Wirbelsäulenlänge (spinal length) während des gesamten Untersuchungszeitraums. Die farbliche Kennzeichnung markiert die drei Untersuchungsgruppen. Die blaue Gruppe umfasst die Messungen der VEPTR ${ }^{\circledR}$-Behandlung; prä- und postoperativ sowie bei Wechsel des Implantats mit MAGEC®_ Einbau. In der roten Gruppe wurden die Messwerte vor und nach MAGEC®-Implantation der Kinder zusammengefasst, die ausschließlich mit einem MAGEC®-Implantat behandelt wurden. Beide Subgruppen wurden nach der MAGEC®-Implantation während der perkutanen Verlängerungen zur grünen Gruppe zusammengefasst. Zu jedem Messwert sind die Standardabweichung und die Anzahl der ausgewerteten Patienten ( $\mathrm{n}=$ vor Verlängerung/nach Verlängerung) angegeben. Die Zeit vom MAGEC $®$-Einbau bis zur ersten Verlängerung umfasste 5 Monate, zwischen den jeweiligen nachfolgenden Verlängerungen lagen 3 Monate. ${ }^{* * *} \mathrm{p}<0,001$ 


\subsubsection{Wirbelsäulenlänge in Abhängigkeit vom Alter}

Wird die Studiengruppe wiederum in zwei Altersgruppen gegliedert, nämlich jeweils unter sechs Jahre (blaue Raute, $n=4)$ oder im Alter von sechs bis zehn Jahren (rotes Quadrat, $n=15$ ) und trägt die Wirbelsäulenlänge prä- und postoperativ und bis zur achten Verlängerung auf, so erhält man die Abbildung 24. Die Grafik zeigt keine deutlichen Unterschiede zwischen den Subgruppen. Die jüngeren Kinder zeigten eine initiale postoperative Zunahme ihrer Wirbelsäulenlänge von $54 \mathrm{~mm}$, die älteren Kinder von $45 \mathrm{~mm}$, was in beiden Fällen ein signifikantes Ergebnis darstellt $(n=4: p<0,01 ; n=15: p<0,001)$. Die Gruppe der älteren Kinder wies im Verlauf im Rahmen jeder Verlängerung hoch signifikante Ergebnisse auf $(\mathrm{p}<0,001)$, während die jüngere Gruppe sehr signifikante Ergebnisse zeigte $(p<0,01)$. In beiden Gruppen allerdings konnte durch die initiale Operation die stärkste Zunahme der Sitzlänge verzeichnet werden. 


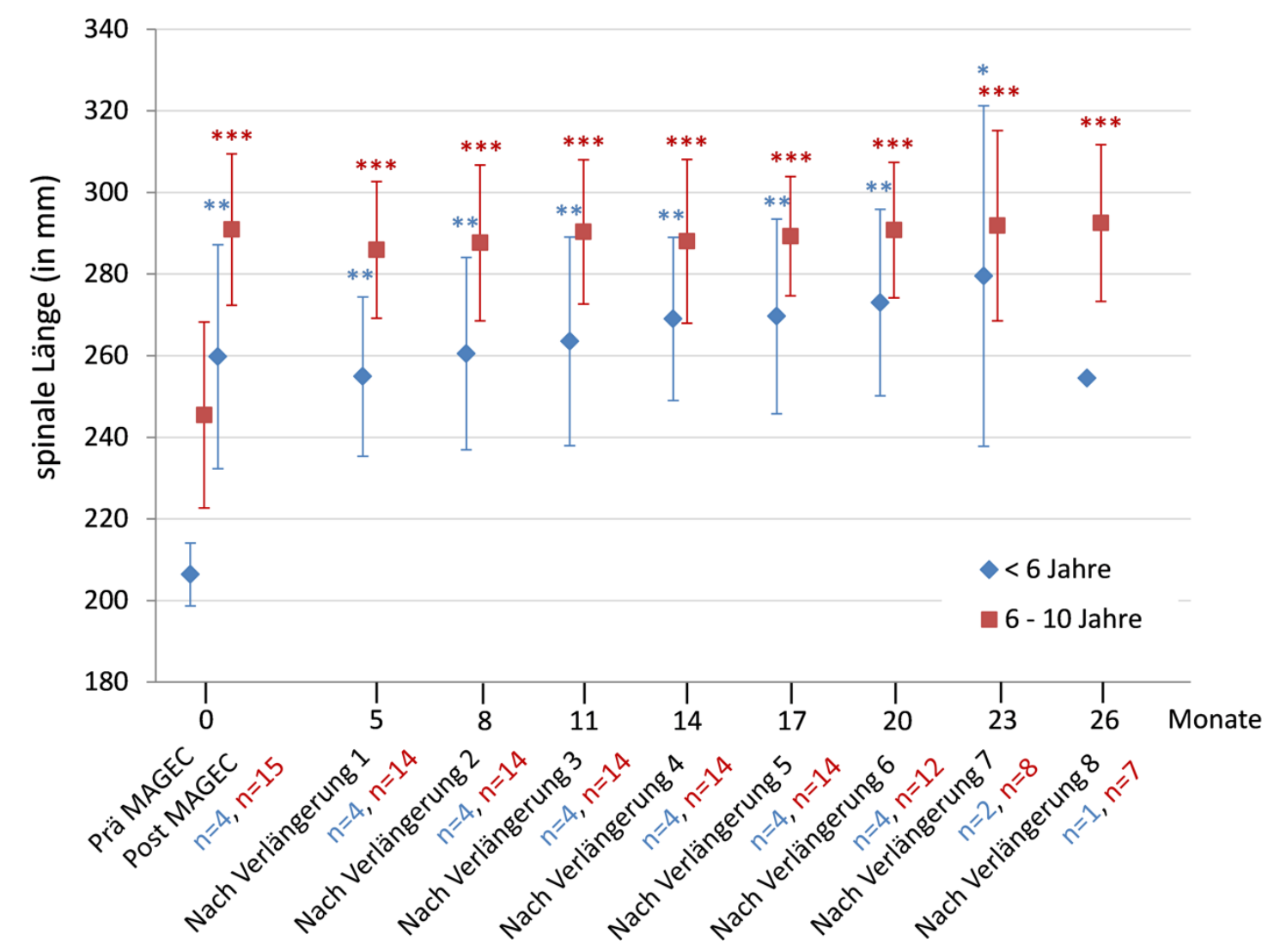

Abbildung 24: Wirbelsäulenlänge gruppiert nach Alter. Dargestellt ist die Wirbelsäulenlänge (spinal length) vor und nach dem MAGEC®-Einbau und nach jeder Verlängerung bis zur 8. Verlängerung, gruppiert nach Alter bei MAGEC®-Einbau. Die blaue Gruppe zeigt die Messwerte der <6-jährigen Kinder, die rote Gruppe der 6-10-jährigen Kinder. Zu jedem Messwert sind die Standardabweichung und die Anzahl der ausgewerteten Patienten (n) angegeben. Die Zeit vom MAGEC®-Einbau bis zur ersten Verlängerung umfasste 5 Monate, zwischen den jeweiligen nachfolgenden Verlängerungen lagen 3 Monate. ${ }^{*} \mathrm{p}<0,05 ;{ }^{* *} \mathrm{p}<0,01 ; * * \mathrm{p}<0,001$

\subsubsection{Wirbelsäulenlänge in Abhängigkeit vom Kinder-BMI}

In Abbildung 25 wurden die Kinder in Gruppen entsprechend ihres BMI eingeteilt, Übergewicht (grünes Dreieck, $n=8$ ), Normalgewicht (rotes Quadrat, $n=5$ ) und Untergewicht (blaue Raute, $\mathrm{n}=8$ ). Auch diese Grafik zum BMI zeigt keine deutlichen Unterschiede zwischen den Subgruppen. Es konnte jedoch gezeigt werden, dass alle Kinder direkt postoperativ eine signifikante Zunahme ihrer Wirbelsäulenlänge erfuhren, welche unterschiedlich stark ausfiel. Waren die Kinder normal- oder untergewichtig, erfuhr ihre Sitzlänge direkt postoperativ eine signifikante Zunahme $(\mathrm{p}<0,05)$, waren sie übergewichtig wurde eine sehr signifikante Zunahme erzielt $(p<0,01)$. 
Es wird hingegen ersichtlich, dass die unter- und übergewichtigen Kinder im Gegensatz zu den Normalgewichtigen beim Endpunkt V6 weiterhin eine sehr signifikante Zunahme $(p<0,01)$ der Wirbelsäulenlänge in Bezug zum präoperativen Ausgangswert aufwiesen.

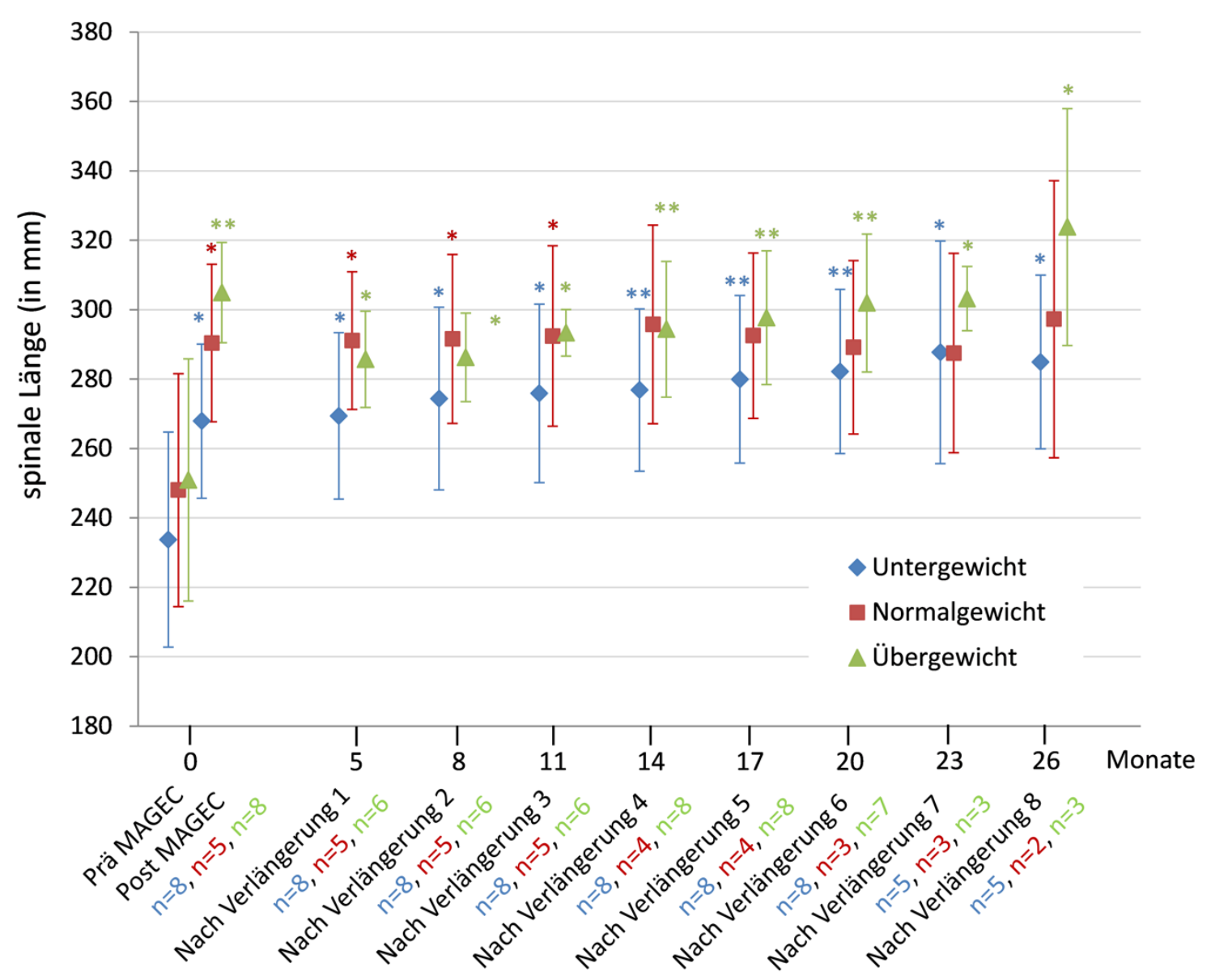

Abbildung 25: Wirbelsäulenlänge gruppiert nach BMI. Dargestellt ist die Wirbelsäulenlänge (spinal length) vor und nach dem MAGEC®-Einbau und nach jeder Verlängerung bis zur 8. Verlängerung, gruppiert nach Kinder-BMI bei MAGEC®-Einbau (Untergewicht=blaue Gruppe; Normalgewicht=rote Gruppe; Übergewicht=grüne Gruppe). Zu jedem Messwert ist die Standardabweichung und die Anzahl der ausgewerteten Patienten (n) angegeben. Die Zeit vom MAGEC®-Einbau bis zur ersten Verlängerung umfasste 5 Monate, zwischen den jeweiligen nachfolgenden Verlängerungen lagen 3 Monate. ${ }^{*} \mathrm{p}<0,05 ;{ }^{* *} \mathrm{p}<0,01$

\subsection{Varianz zwischen den Untersuchern}

Die Messwerte wurden unabhängig voneinander von zwei Untersuchern erhoben. Aus den erhobenen Messwerten wurde ein Mittelwert gebildet, der die Grundlage für die Ergebnisse bildete. Um die Messgenauigkeit zwischen den beiden Untersuchern darzustellen, wurde eine Analyse zur Interobserver-Abweichung durchgeführt. 


\subsubsection{Varianz des Skoliosekrümmungswinkels (Cobb-Winkel)}

Die Abbildung 26 stellt eine beispielhafte Varianz zwischen den Untersuchern anhand der Messung des Skoliosekrümmungswinkels prä- und postoperativ und jeweils nach den Verlängerungsprozeduren bis zur siebten Verlängerung dar. Aus diesem Graphen und den dazugehörigen Rohdaten wird ersichtlich, dass die durchschnittliche Messabweichung zwischen beiden Untersuchern bei $1,03^{\circ} \operatorname{lag}\left(0,10^{\circ}-2,76^{\circ}\right)$.

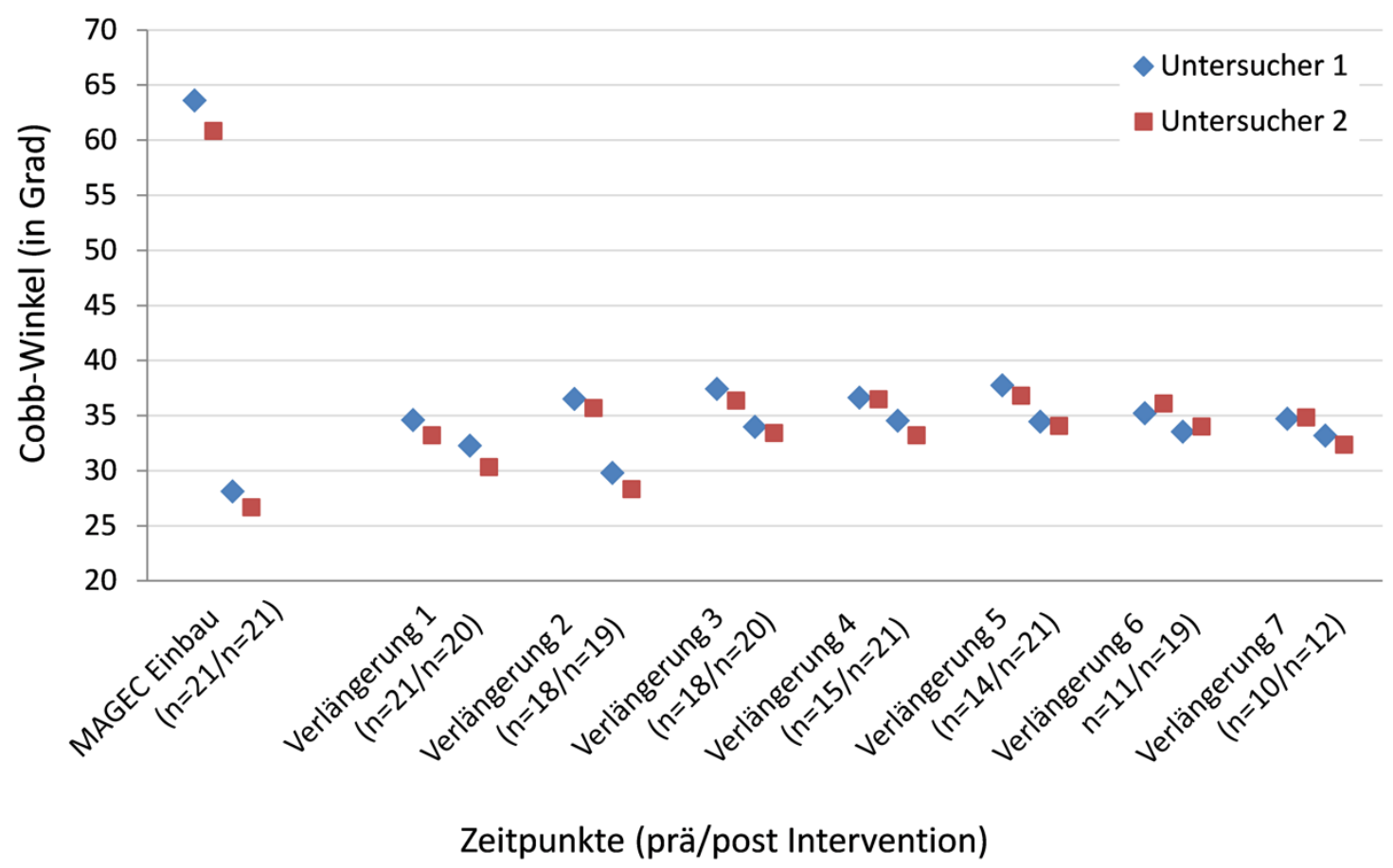

Abbildung 26: Varianz zwischen den Untersuchern anhand des Skoliosekrümmungswinkels (CobbWinkel). Dargestellt ist der Skoliosekrümmungswinkel bis zur 7. Verlängerung. Die farbliche Kennzeichnung markiert die beiden Untersucher. Zu jedem Messwert ist die Anzahl der ausgewerteten Patienten ( $\mathrm{n}=$ vor Verlängerung/nach Verlängerung) angegeben. Die Zeit vom MAGEC®-Einbau bis zur ersten Verlängerung umfasste 5 Monate, zwischen den jeweiligen nachfolgenden Verlängerungen lagen 3 Monate.

\subsection{Komplikationen}

Bei insgesamt 325 analysierten Interventionen (21 Primäroperationen und 304 ambulante Verlängerungen) fanden sich insgesamt 21 Komplikationen, was einer Komplikationsrate von 6,5\% entspricht. Hiervon wurden vier perioperative (19\%) und 17 implantat-assoziierte Komplikationen (5,6\%) verzeichnet. Es konnte $13 \mathrm{Mal}$ der Stab nicht regelrecht distrahiert werden (4,3\%). Alle Komplikationen führten in drei Fällen zu einer operativen Revision. 


\subsubsection{Perioperative Komplikationen}

Die ausgewerteten perioperativen Komplikationen umfassen folgende Komplikationen, die einerseits dem allgemeinen Operationsrisiko entsprechen und andererseits spezifische Komplikationen im Rahmen des operativen Implantateinbaus darstellen.

- Pneumothorax, Embolie, Wundheilungsstörungen, Frühinfekt, Druckulzera, postoperative Schmerzen länger als drei Monate

Im untersuchten Kollektiv konnten insgesamt vier solcher Komplikationen gefunden werden (s. Tabelle 1). Ein Kind erlitt eine Wundinfektion nach dem Einbau, die mit intravenöser Antibiotikatherapie und Debridement ohne Implantatausbau gut kontrolliert werden konnte. Ein Kind erlitt durch die Operation einen Pneumothorax, welcher mittels Bülaudrainage therapiert wurde. Bei einem weiteren Kind musste aufgrund einer Stabdislokation in Verbindung mit einer Rippenfraktur und einem Pneumothorax eine Revisionsoperation erfolgen. Ein Kind litt an postoperativen Schmerzen von einer Dauer von mehr als drei Monaten.

Die Komplikationen Embolie, Frühinfekt und perioperative Druckulzera traten bei keinem der an der Studie teilnehmenden Kinder auf.

\subsubsection{Implantat-assoziierte Komplikationen}

Die implantat-assoziierten Komplikationen umfassen folgende Komplikationen:

- Spätinfekt, Implantatversagen- bzw. Implantatbruch, Dislokation, Nichtdistrahierbarkeit, Rippenfraktur

Insgesamt fanden sich 17 implantat-assoziierte Komplikationen. Die am häufigsten $(n=13)$ zu verzeichnende Komplikation fand sich in der Nichtdistrahierbarkeit, bzw. nicht regelrechten Distrahierbarkeit wieder. Bei fünf Kindern trat diese Komplikation auf. Drei Kinder erfuhren dieses Problem einmalig, wobei darunter in einem Fall der Stab zu stark ausgefahren wurde, einmalig keine Distraktion und einmalig eine inkomplette Distraktion stattfand. Von den genannten fünf Kindern konnte bei einem Kind zweimalig das Implantat nicht ausgefahren werden. Das fünfte Kind erfuhr in seinen fünf Verlängerungsprozeduren lediglich zweimalig 
die Verlängerung eines Stabes, sodass es nach der nicht erfolgreichen fünften Distraktion eine operative Distraktion des MAGEC®-Systems bekam. Drei dieser fünf Kinder, bei denen das Implantat nicht regelrecht ausgefahren werden konnte, wiesen einen BMI von über 23 auf, entsprechend der 89., 91. und 97. Perzentile.

Ein Kind litt im Verlauf an einem Druckulcus über dem kranialen Rippenhaken. In zwei Fällen konnte eine Implantatwanderung mit Rippenfraktur verzeichnet werden, die in einem Fall zur Dislokation des Stabsystems führte. Ein weiteres Mal wurde eine Diskonnektion des Querauslegers beobachtet. Der Querausleger wurde bei einigen Kindern verwendet, um eine höhere Belastbarkeit des kranialen Rippenhakens zu erzielen.

\subsubsection{Studienabbruch}

Im Verlauf verließ kein Kind aufgrund von persönlichen oder medizinischen Gründen die Studie.

\subsection{Zusammenfassung der Ergebnisse}

In der Auswertung der erhobenen Daten fanden sich die folgenden Ergebnisse für Kinder mit Wirbelsäulendeformität und SMA, die mit einem kombinierten VEPTR®/MAGEC®Implantat behandelt wurden:

\section{Skoliosekrümmungswinkel (Cobb-Winkel)}

Veränderung des Skoliosekrümmungswinkels (Cobb-Winkels) zum Zeitpunkt des MAGEC®_ Einbaus:

- Gesamtgruppe ( $\left.\mathrm{n}=16 ; 61,3^{\circ} \mathrm{zu} 27,1^{\circ}\right)$ : Signifikante Reduktion um 55,9\% $(\mathrm{p}<0,001)$

- Gruppe der spinal voroperierten ( $\left.\mathrm{n}=4 ; 30,8^{\circ} \mathrm{zu} 18,1^{\circ}\right)$ : Reduktion um $41,2^{\%}$

- Gruppe der Primärimplantierten ( $\mathrm{n}=17 ; 69,6^{\circ} \mathrm{zu} 29,6^{\circ}$ ): Signifikante Reduktion um $57,5 \%(\mathrm{p}<0,001)$

Veränderung des Skoliosekrümmungswinkels nach 20 Monaten follow-up (6. Verlängerung):

- Gesamt ( $\left.\mathrm{n}=16 ; 61,3^{\circ} \mathrm{zu} 32,7^{\circ}\right)$ : Signifikante Reduktion um 46,7\% $(\mathrm{p}<0,001)$ zum präoperativen Wert 


\section{Beckenschiefstand (pelvic obliquity)}

Veränderung des Beckenschiefstandes zum Zeitpunkt des MAGEC®-Einbaus:

- Gesamtgruppe ( $\mathrm{n}=17 ; 14,2^{\circ}$ zu $\left.4,4^{\circ}\right)$ : Signifikante Reduktion um 69\% $(\mathrm{p}<0.001)$

- Gruppe der spinal voroperierten $\left(\mathrm{n}=4 ; 5,6^{\circ} \mathrm{zu} 3,3^{\circ}\right)$ : Reduktion um 37,5\%

- Gruppe der Primärimplantierten ( $\mathrm{n}=17 ; 16,8^{\circ} \mathrm{zu} 4,2^{\circ}$ ): Signifikante Reduktion um $75 \%(\mathrm{p}<0,001)$

Veränderung des Beckenschiefstandes nach 20 Monaten follow-up (6. Verlängerung):

- Gesamt ( $\mathrm{n}=17 ; 14,2^{\circ}$ zu 5,90): Signifikante Reduktion um 58,5\% $(\mathrm{p}<0,001)$

\section{Thorakale Kyphose und lumbale Lordose}

Sowohl die thorakale Kyphose als auch die lumbale Lordose konnten durch den Einbau des MAGEC®-Systems und im Verlauf nicht umfassend kontrolliert werden. Bei der Kyphose zeigte sich ein Trend zur thorakalen Hyperkyphose, lumbal ein Trend zur relativen Abflachung des sagittalen Profils.

\section{Wirbelsäulenlänge (spinal length)}

Veränderung der Wirbelsäulenlänge zum Zeitpunkt des MAGEC®-Einbaus:

- Gesamtgruppe (n=9; $243 \mathrm{~mm}$ zu $276 \mathrm{~mm}$ ): Signifikante Zunahme um 13,6\% $(\mathrm{p}<0,001)$

- Gruppe der spinal voroperierten ( $\mathrm{n}=4 ; 287 \mathrm{~mm}$ zu $300 \mathrm{~mm}$ ): Zunahme um $4,5 \%$

- Gruppe der Primärimplantierten ( $\mathrm{n}=17 ; 234 \mathrm{~mm} \mathrm{zu} 285 \mathrm{~mm}$ ): Signifikante Zunahme um 21,8\% $(\mathrm{p}<0,001)$

Veränderung der Wirbelsäulenlänge im ersten Jahr (vor 1. Verlängerung bis nach 4. Verlängerung):

- Gesamt ( $\mathrm{n}=18: 276 \mathrm{~mm}$ zu n=20: $288 \mathrm{~mm}):$ Zunahme um $12 \mathrm{~mm} / \mathrm{Jahr}$ 


\section{Interobserver-Variabilität}

Die durchschnittliche Varianz zwischen beiden Untersuchern, hier beispielhaft am Skoliosekrümmungswinkel errechnet, belief sich auf durchschnittlich $1,03^{\circ}\left(0,10^{\circ}-2,76^{\circ}\right)$.

\section{Komplikationsrate}

Es ergab sich eine Komplikationsrate von 6,5\% ( $\mathrm{n}=21$ bezogen auf 325 Interventionen, davon $\mathrm{n}=21$ operative Implantationen und n=304 ambulante Verlängerungen). Darunter fanden sich vier perioperative (19\%) und 17 implantat-assoziierte Komplikationen (5,6\%). In drei Fällen wurde eine Revisionsoperation erforderlich.

Betrachtet man alleine die Verlängerungsprozeduren, so wurden also 304 Implantate verlängert, wovon 13 Mal der Stab nicht distrahiert werden konnte. Dies entspricht einer Rate von $4,3 \%$. 


\section{Diskussion}

Kinder mit spinaler Muskelatrophie entwickeln frühzeitig gravierende Wirbelsäulendeformitäten. Diese early-onset-Skoliose ist progredient und einer konservativen Therapie nicht zugänglich (Sucato 2007). Wegen der überaus raschen Progredienz ist eine operative Therapie für diese Kinder in den allermeisten Fällen unumgänglich. Ansonsten ist früh die Entstehung einer nicht kontrollierbaren Krümmung zu erwarten, die mit Problemen wie dem Thoraxinsuffizienz-Syndrom, pulmonalen Komplikationen bis hin zum frühen Tod einhergehen kann (Akbarnia et al. 2011; Hell et al. 2005; Tis et al. 2012). Da sich die Kinder im Wachstum befinden, muss in der operativen Therapie diesem Wachstum Rechnung getragen werden (Tis et al. 2012). Daher ist ein Konstrukt nötig, das das Wachstum ermöglicht, indem es beispielsweise durch Teleskopstäbe im Intervall verlängert werden kann. Es ist bekannt, dass Kinder mit einer neuromuskulären Skoliose höhere Komplikationsraten (Master et al. 2011; Brunner und Gebhard 2002) und verlängerte stationäre Aufenthalte aufweisen (McElroy et al. 2011). Besonders die pulmonalen Probleme von an SMA erkrankten Kindern stellen eine schwierige Situation für wiederholte operative Therapien dar. In den letzten Jahrzehnten wurden viele growth friendly spinal implants angewandt (Campbell et al. 2004a; Tis et al. 2012; Skaggs et al. 2014), beispielsweise in Form eines VEPTR ${ }^{\circledR}$, die in den meisten Fällen allerdings eine operative Verlängerung des Teleskopstabsystems alle sechs Monate erforderten. Um die Anzahl an Operationen und damit zusammenhängende Risiken und Komplikationen zu reduzieren, wurden in den letzten Jahren magnetische Implantate bzw. magnetically controlled growing rods (MCGR) etabliert (z. B. MAGEC®, Ellipse Technologies, Irvine, Inc., California, USA). Im Jahre 2009 implantierten Cheung et al. erstmals einem Kind ein magnetisches Stabsystem (Cheung et al. 2012). So findet sich in der Literatur eine wachsende Anzahl von frühen Zwischenberichten einer MCGR-Therapie (Cheung et al. 2012; Akbarnia et al. 2013; La Rosa et al. 2017; Lebon et al 2017). Die meisten dieser Studien berichten jedoch von heterogenen Patientenkollektiven, kleiner Fallzahl und kurzem Beobachtungszeitraum.

Nach der von Hell et al. (2018) beschriebenen Methode mit bilateralen extern zu steuernden magnetischen Implantaten wurde bisher nur ein weiterer Fall veröffentlicht (Kwan et al. 2018). Durch die paravertebrale Rippen-zu-Beckenkamm-Verankerung entfällt die spinale Befestigung mittels Pedikelschrauben. Dadurch werden beschriebene Komplikationen wie Ossifikationen und Autofusion umgangen (Akbarnia und Emans 2010; Grönefeld und Hell 
2013), die eine letztliche operative Versorgung erschweren würden (Lattig et al. 2012). Möglicherweise kann durch die kurzen Abstände der Verlängerungen eine zunehmende Wirbelsäulensteifigkeit verhindert werden (Choi et al. 2017), wodurch die darauffolgende Spondylodese unkomplizierter verlaufen würde (Hasler et al. 2010; Akbarnia und Emans 2010).

In der vorliegenden prospektiven Untersuchung wurde erstmals ein homogenes Kollektiv von 21 Kindern mit SMA und Wirbelsäulendeformität untersucht, das mittels eines kombinierten VEPTR $® / M A G E C \circledR-K o n s t r u k t s$ operativ versorgt wurde und ein einheitliches Nachuntersuchungsschema mit einem follow-up von zwei Jahren aufwies. Allen Kindern wurde ein bilaterales magnetisches Stabsystem implantiert, das an Rippen und Beckenkamm fixiert wurde. Erstmals wurde das System fünf Monate postoperativ um jeweils $5 \mathrm{~mm}$ distrahiert, in der Folge alle drei Monate um ebenfalls $5 \mathrm{~mm}$. Vier der Kinder waren im Sinne einer VEPTR ${ }$-Versorgung spinal voroperiert.

\subsection{Korrektur der Wirbelsäulendeformität und des Beckenschiefstandes}

Im untersuchten Kollektiv konnte gezeigt werden, dass extern zu steuernde, transkutan distrahierbare growth friendly spinal implant-Systeme, hier in Form eines kombinierten VEPTR ${ }^{\circledR} /$ MAGEC ${ }$-Systems, sehr gut geeignet sind, die Skoliose in der Frontalebene bei SMA initial zu korrigieren. Der präoperative durchschnittliche Skoliosekrümmungswinkel betrug in diesem Kollektiv durchschnittlich $62^{\circ}$, in der nicht voroperierten Gruppe $70^{\circ}$, was vergleichbar mit anderen Studien ist (Akbarnia et al. 2013 60 ; Ridderbusch et al. 2017 63;

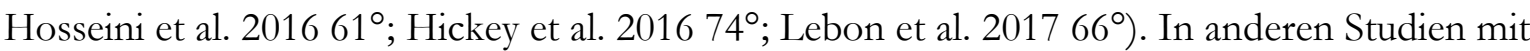
heterogenem Patientenkollektiv konnte eine Primärkorrektur von 54\% (Ridderbusch et al. 2017), zweimalig 43\% (Akbarnia et al. 2013; Hickey et al. 2014), 39,4\% (Lebon et al. 2017) und 44\% (Hosseini et al. 2016) gezeigt werden. Das vorliegende Kollektiv ( $\mathrm{n}=21$ ) wies im Vergleich eine sehr gute Primärkorrektur von 56\% (62 $\left.{ }^{\circ} \mathrm{zu} 28^{\circ}\right)$ auf. Die Gruppe der Kinder ohne vorherige spinale Operation $(\mathrm{n}=17)$ ließen sich um $57 \%\left(70^{\circ} \mathrm{zu} 30^{\circ}\right)$ korrigieren, während der Skoliosekrümmungswinkel der voroperierten Kinder, bei denen ein Implantatwechsel stattfand, um $42 \%$ reduziert wurde $\left(\mathrm{n}=4,31^{\circ} \mathrm{zu} 18^{\circ}\right)$.

Im Literaturvergleich konnten mit dem in dieser Arbeit untersuchten MAGEC®-Implantat bessere Primärkorrekturen erzielt werden als in Studien, die ein reines VEPTR®-System an gemischten Patientenkollektiven untersuchten. Beispielsweise beschrieben Wimmer et al. 2010 eine Korrektur mittels VEPTR ${ }^{\circledR}-S y s t e m$ von 51\% ( $\mathrm{n}=39,11$ neuromuskuläre Skoliosen), 
Hasler et al. beschrieben 2010 eine Initialkorrektur von 29,4\% ( $n=23 ; 11$ neuromuskuläre Skoliosen) und Samdani et al. 2009 von 38,1\% (n=11; 4 neuromuskuläre Skoliosen).

Während des Beobachtungszeitraums konnte die Korrektur des Skoliosekrümmungswinkels des vorliegenden Kollektivs aufrechterhalten werden. So konnte auch nach 20 Monaten weiterhin eine signifikante Reduktion $(\mathrm{p}<0,001)$ des Winkels verzeichnet werden, die dem postoperativen Wert entsprach. Diese Ergebnisse zeigen erstmals auf, dass die flexible Wirbelsäulendeformität im Zusammenhang mit SMA durch das hier verwendete bilaterale magnetische Implantat, an Rippen und Beckenkamm fixiert, initial gut korrigiert und diese Korrektur auch im Verlauf erhalten werden kann. Ferner wurde beobachtet, dass jüngere Kinder unter sechs Jahren hier eine stärkere Korrektur nach 20 Monaten von 66\% aufwiesen, als ältere Kinder zwischen sechs und zehn Jahren (39\%). Denkbar ist, dass die Wirbelsäule der älteren Kinder weniger flexibel ist oder ein größeres Körpergewicht bei den älteren Kindern die Therapie erschwert. Um eine bessere Aussage über diesen Effekt machen zu können, wären weitere Untersuchungen notwendig, da die Subgruppe der jüngeren Kinder lediglich vier Patienten umfasste.

Neben dem Skoliosekrümmungswinkel wurden auch hinsichtlich des Beckenschiefstandes signifikante Ergebnisse verzeichnet. Die Lebensqualität der SMA-Kinder hängt stark von der Beckenstellung und damit der Sitzposition im Rollstuhl ab. Indirekt wird hierdurch auch die Kopfkontrolle beeinflusst. Es konnte gezeigt werden, dass der Beckenschiefstand durch das MAGEC®-Implantat initial deutlich abnahm (75\%), mit der Tendenz gegen Ende des Untersuchungszeitraums nicht signifikant geringfügig anzusteigen. Diese Ergebnisse implizieren, dass das hier verwendete an Rippen und Beckenkamm fixierte bilaterale magnetische Stabsystem sehr gut geeignet ist, um den Beckenschiefstand und somit die Sitzposition der SMA-Kinder zu verbessern. In einer Studie von Hasler et al. (2010) wurde ebenfalls der Beckenschiefstand im Rahmen einer VEPTR ${ }^{\circledR}$-Behandlung untersucht, jedoch erst ab einer Größe von $>10^{\circ}$. Hasler et al. (2010) stellten fest, dass in ihrem heterogenen Patientenkollektiv Beckenschiefstände von über $30^{\circ}$ lediglich um $20 \%$ korrigiert werden konnten, während jene unter $30^{\circ}$ um $80 \%$ korrigiert wurden. Daher empfahlen Hasler et al. (2010) in schwierigen Fällen eine Fixierung im Sinne eines Eiffeltower-Konstrukts; eine Rippenzu-Becken-Fixierung.

Allerdings ist die Ermittlung des Beckenschiefstands stark sitzpositionsabhängig. Grundsätzlich ist die Durchführung von standardisierten Röntgenaufnahmen der Wirbelsäule bei nicht stehfähigen Kindern schwierig. Um eine größtmögliche Standardisierung zu erzielen, wurden die Patienten des vorliegenden Studienkollektivs in einem röntgendurchlässigen Stuhl 
sitzend untersucht. Somit wurde eine einheitliche Sitzposition für die Wirbelsäulenaufnahme mit der Belastung durch das Sitzen erreicht. Jedoch ist die Standardisierung nicht gänzlich vergleichbar mit einer Stehendaufnahme.

\subsection{Sagittales Profil- Kyphose, Lordose und Wirbelsäulenlänge}

Hinsichtlich des sagittalen Profils führte die Implantation des kombinierten VEPTR ${ }^{\circledR} /$ MAGEC ${ }^{\circledR}$-Konstrukts nicht zu einer suffizienten Kontrolle der thorakalen Kyphose. Präoperativ wies das Patientenkollektiv eine vermehrte altersadaptierte Kyphose von $18,8^{\circ}$ auf. Diese Hyperkyphose konnte durch das MAGEC®-Implantat nicht signifikant verbessert werden. So betrug die altersadaptierte Kyphose direkt postoperativ 6,4 $4^{\circ}$ und nahm im Verlauf nach 20 Monaten wieder zu $\left(11,6^{\circ}\right)$. Ähnliche Zunahmen wurden auch in anderen Studien beschrieben (Akbarnia et al. 2013; Samdani et al. 2009; Hosseini et al. 2016; Lebon et al. 2017; Ridderbusch et al. 2017; Gantner et al. 2018). Als Grund für die Zunahme wird einerseits diskutiert, dass als Komplikation die Anschlusskyphose bzw. proximal junctional kyphosis (PJK) auftreten kann (Thompson et al. 2016). In der Literatur ist keine einheitliche Definition der PJK etabliert. Lau et al. (2014) sowie Lee und Park (2016) beschrieben die PJK als eine im Rahmen der Wirbelsäulenchirurgie auftretende Komplikation, die asymptomatisch oder in Form von Schmerzen, Problemen beim Laufen und neurologischen Defiziten auffallen kann bzw. sich kosmetisch präsentiert. Die PJK beschreibt demnach eine kyphotische Deformität, die am proximalen Ende der Instrumentierung auftritt und Revisionsoperationen erforderlich machen kann (Lau et al. 2014). Die Entwicklung einer PJK wird in dieser Arbeit lediglich diskutiert, sie war kein Gegenstand der Fragestellung und radiologischen Auswertung. Grund hierfür ist die mangelnde Kopfkontrolle der SMA-Kinder, was eine standardisierte reelle Erfassung nicht möglich machte. Trotzdem haben Kinder ohne Kopfkontrolle grundsätzlich ein erhöhtes Risiko eine thorakale Hyperkyphose zu entwickeln. Um diesen Effekt in der vorliegenden Arbeit zu minimieren, wurde lediglich die Kyphose im Bereich der Instrumentierung ermittelt. Trotzdem kann der Einfluss der mangelnden Kopfkontrolle auf die thorakale Kyphose in dieser Studie nicht vollständig ausgeschlossen werden.

Weiterhin zeigten die Ergebnisse, dass im Verlauf eine Hypolordose (- $7^{\circ}$ Abweichung des altersabhängigen Normwerts bei Verlängerung 6) auftrat. Diese entstand jedoch allem Anschein nach nicht durch die Behandlung mittels des MAGEC®-Implantats per se, jedoch durch die retrograde Kippung des Beckens im Rahmen der Rippen-zu-BeckenkammFixierung. 
Es konnte überdies gezeigt werden, dass die Wirbelsäulenlänge durch die Implantation um 51 mm bzw. 21,8\% zunahm, was den Ergebnissen anderer Studien am MAGEC®-Implantat von Lebon et al. (2017) mit $59 \mathrm{~mm}$ und La Rosa et al. (2017) mit $57 \mathrm{~mm}$ entspricht. Nach der initialen Zunahme der Wirbelsäulenlänge wurde eine weitere Längenzunahme von $12 \mathrm{~mm}$ im ersten Jahr beobachtet. Dieser Wert entspricht in etwa dem Wirbelsäulenwachstum von gesunden Kindern derselben Altersgruppe mit $10 \mathrm{~mm}$ pro Jahr (Canavese und Dimeglio 2013). Die angestrebte Distraktion pro Verlängerungsprozedur betrug $5 \mathrm{~mm}$, bei vier Verlängerungen im Jahr würde dies einer Zunahme von $20 \mathrm{~mm}$ entsprechen. Die Diskrepanz zu der tatsächlich gemessenen Zunahme der Wirbelsäulenlänge ist vermutlich in einer Implantatwanderung begründet. Diese wurde im Zusammenhang mit VEPTR®-Implantaten bereits beschrieben (Campbell und Smith 2007; Groenefeld und Hell 2013). Auch bei magnetischen Implantaten wurde diese Diskrepanz bereits dokumentiert (Lebon et al. 2017). Die regelmäßigen Verlängerungen halten ein gewisses $\mathrm{Maß}$ an Spannung innerhalb des Implantats aufrecht. Da die Rippen bei Kindern eine große Flexibilität aufweisen, überträgt sich die Verlängerungsdistanz nicht gänzlich auf die Wirbelsäule. Bei diesem Implantat sind sowohl die Rippen- als auch die Beckenverankerungen flexibel. Durch die stetigen Bewegungen sinken die Beckenhaken in die Beckenschaufel ein, während die Rippenhaken nach kranial wandern. Dadurch soll zum einen ein hohes $\mathrm{Maß}$ an Wirbelsäulenflexibilität erhalten bleiben, zum anderen führen die stetigen Bewegungen im Bereich der Rippen- und Beckenhaken zu einer Implantatwanderung. In Extremfällen können die Rippenhaken komplett durch die Rippen durchwandern und dislozieren und die Beckenhaken mehrere Zentimeter in die Beckenschaufel absinken. Weiterhin ist denkbar, dass sich die interindividuellen Intervalle zwischen den Verlängerungen, beispielsweise aufgrund von Erkrankung, negativ auswirken und die Wirbelsäule weniger flexibel ist und sich schlechter verlängern lässt. Sankar et al. (2011) beschrieben eine Hypothese, wonach mit Zunahme der Distraktionen die Zunahme der Wirbelsäulenlänge pro Prozedur abnimmt - „law of diminishing returns". Entsprechende Ergebnisse ergaben sich auch in einer Studie von Noordeen et al. (2011). In der vorliegenden Studie konnte dieses Phänomen am ehesten aufgrund von kurzen, regelmäßigen Abständen zumindest in den ersten beiden Jahren nicht beobachtet werden. Es wird überdies diskutiert, ob diese kürzeren Verlängerungsabstände eine zunehmende Wirbelsäulensteifigkeit verhindern können (Choi et al. 2017) Cheung et al. (2018) beobachteten in ihrer Studie erst eine Abnahme der antizipierten Distraktionslänge bei zunehmender Einsatzdauer eines MCGR, und zwar nach Erreichen von mehr als der Hälfte der zugelassenen Stablänge. Dies trat im vorliegenden Kollektiv nicht auf und ist am ehesten auf technische Probleme der Implantate der ersten Generation zurückzuführen. 


\subsection{Korrektur der Wirbelsäulendeformität im Verlauf}

Im Verlauf nach 20 Monaten follow-up konnte die Korrektur der Skoliose aufrechterhalten werden. So fand sich im primärimplantierten Patientenkollektiv $(n=15)$ nach der sechsten Verlängerung immer noch eine Korrektur von 56\%, womit sie also lediglich um $1 \%$ zum postoperativen Wert abnahm. Diese unwesentliche Veränderung des direkt postoperativ ermittelten Winkels bei MAGEC®-Implantation ist mit anderen Studien vergleichbar. So gaben Thompson et al. (2016) in einem heterogenen Studienkollektiv ( $\mathrm{n}=19)$ direkt postoperativ eine Verminderung von 27,3\% und nach 22,4 Monaten von 29,6\% an. Andere Arbeitsgruppen, die das Korrekturpotential des VEPTR ${ }^{\circledR}-$ Systems untersuchten, konnten ähnliche Ergebnisse feststellen. Im direkten Vergleich mit den Daten der vorliegenden Studie wiesen diese jedoch einen etwas größeren Korrekturverlust während des Untersuchungszeitraums auf. So nahm der Skoliosekrümmungswinkel nach Initialkorrektur im Verlauf 7\% (Emans et al. 2005), 5\% (Hasler et al. 2010), bzw. 8\% (Samdani et al. 2009) zu. Am Endpunkt zeigten sie jedoch ebenfalls eine signifikante Verbesserung zum Ausgangswert.

In Bezug auf den Skoliosekrümmungswinkel ist zudem die Messgenauigkeit zwischen den Untersuchern hervorzuheben, die sich in dieser Studie auf $1,03^{\circ}$ belief. In einer Studie von Morrissy et al. (1990) wurde die Messgenauigkeit des Skoliosekrümmungswinkels beispielsweise mit $2,03^{\circ}$ angegeben.

\subsubsection{Komplikationsrate}

Im untersuchten Kollektiv fand sich mit einer Nachuntersuchungszeit von durchschnittlich 24,8 Monaten (17 Monate bis 40,6 Monate) und bei 325 Interventionen eine Komplikationsrate von 6,5\%. In diesen Eingriffen, die sowohl den Einbau als auch die folgenden Verlängerungen umfassen, wurden 21 Komplikationen verzeichnet, unter denen dreimalig eine Revisionsoperation erforderlich wurde. Die Komplikationsrate fiel niedriger aus als in anderen vergleichbaren Studien (Lebon et al. 2017; Hosseini et al. 2016). Die häufigste Komplikation fand sich in Form der Nichtdistrahierbarkeit wieder, die im Verlauf jedoch mit Zunahme an Expertise und Erfahrung durch Einsatz von beispielsweise eines Hypomochlions bei Distraktion weitestgehend behoben werden konnte. Des Weiteren ist der Aspekt des Übergewichts zu diskutieren (s. 3.8.2 Implantat-assoziierte Komplikationen). Denkbar ist, dass der externe Magnet die relativ größere Strecke zum Implantat nicht überwinden konnte. In einem Fall befanden sich beide interne Magneten derart nah beieinander, dass sie von extern nicht gezielt angesteuert werden konnten und somit eine zu große Distraktion als geplant 
erfolgte. Cheung et al. (2018) benannten in ihrer Studie zur Distraktion des Stabsystems eines MCGR und Einflussgrößen diesbezüglich ebenfalls diese Gründe.

Die niedrigere Komplikationsrate kann auf die geringere Invasivität des gesamten Verfahrens zurückgeführt werden. Auf die initiale Operation folgten nicht-invasive Verlängerungsprozeduren, die in regelmäßigen dreimonatigen Abständen ambulant durchgeführt wurden. Die ohnehin gefährdeten Lungen der SMA-Kinder wurden somit einer Narkose und einem stationären Aufenthalt entzogen, wodurch nicht nur implantat-assoziierte Komplikationen niedrig gehalten, sondern vor allem auch ein Gewinn der Lebensqualität verzeichnet werden konnte, was auch in anderen Studien berichtet wurde (Thompson et al. 2016; La Rosa et al. 2017; Cheung et al. 2012).

\subsection{Limitationen, Schlussfolgerung und Ausblick}

Im Rahmen dieser Studie erfolgte ein follow-up von 20 Monaten. Die sechste Verlängerungsprozedur wurde als Endpunkt definiert, da sich das Studienkollektiv zu diesem Zeitpunkt ( $\mathrm{n}=19)$ unwesentlich von dem präoperativen Kollektiv unterschied $(\mathrm{n}=21)$. So blieb aufgrund des prospektiven Charakters bisher die Evaluation von Langzeitfolgen- und Komplikationen dieses Kollektivs aus, was für das volle Verständnis der Anwendung von MCGR von Vorteil wäre.

Der Implantatausbau und die darauffolgende Spondylodese fanden in dieser Arbeit ebenfalls keinen Raum. Hier wird im Folgenden interessant sein, ob sich bei den Wirbelsäulen im Jugendlichenalter eine höhere Flexibilität mit besseren operativen Ergebnissen zeigt als bisher.

Weiterhin wurde die implantat-assoziierte Komplikation der entstehenden Metallose nicht erörtert. Die Metallose beschreibt Gewebeschäden, die infolge des umliegenden Implantats entstehen (Teoh et al. 2016). Diese Komplikation wurde bei Explantation bzw. Revisionsoperationen beobachtet (Teoh et al. 2016), wobei die Auswirkungen auf den kindlichen Organismus diesbezüglich noch nicht bekannt sind (Rushton et al. 2017). Da es sich bei dem vorliegenden Studienkollektiv ausschließlich um Kinder handelt, ist aber insbesondere hier eine genaue Kenntnis der unerwünschten Wirkungen und Komplikationen der Therapie erforderlich. Dies kann mit Zunahme des Beobachtungszeitraums, Einschluss von Daten über den Implantatausbau und eine enge Beobachtung und Protokollierung über jedwede Komplikation erfolgen.

Es konnte gezeigt werden, dass extern zu kontrollierende magnetische Implantate ein geeignetes Mittel sind, um flexible Wirbelsäulendeformitäten bei Kindern mit SMA primär zu 
korrigieren und die Ergebnisse im Zweijahresverlauf zu erhalten bei vergleichsweise geringer Komplikationsrate. Die Stärke dieser Studie liegt in ihrem prospektiven Charakter mit einem homogenen Patientenkollektiv, einheitlicher Operationstechnik sowie standardisiertem Verlängerungs- und Nachuntersuchungsprotokoll. 


\section{$5 \quad$ Zusammenfassung}

Kinder mit SMA (Spinaler Muskelatrophie) Typ I und II entwickeln in jungen Jahren progrediente Wirbelsäulendeformitäten. Wegen der eingeschränkten Lungenfunktion sind konservative Maßnahmen nicht möglich, so dass schon frühzeitig operative Interventionen diskutiert werden müssen. In den vergangenen Jahrzehnten wurden verschiedene wachstumsfreundliche Implantate für Kinder mit Wirbelsäulendeformitäten entwickelt. Diese müssen im Allgemeinen alle sechs Monate operativ verlängert werden und weisen eine hohe Komplikationsrate auf. Die 2009 erstmals am Kind implantierten extern zu steuernden magnetischen Wirbelsäulenimplantate reduzieren die Anzahl der operativen Eingriffe, da notwendige Verlängerungen ohne invasiven Eingriff durchgeführt werden können. An der UMG wurde 2011 eine Operationsmethode entwickelt, welche die Vorteile einer beidseitigen, nicht wirbelsäulenbasierten Implantatverankerung des VEPTR ${ }^{\circledR}$ (vertical expandable prosthetic titanium rib)-Systems mit den neuartigen Magnetstäben (MAGEC®) kombiniert. Ziel der vorliegenden Untersuchung war die Evaluation eines prospektiven homogenen Kollektivs von SMA-Kindern mit Wirbelsäulendeformität, welches mit diesem Implantat versorgt worden ist und in der Folge mehrfach verlängert wurde.

Das Studienkollektiv ( $\mathrm{n}=21$ ) umfasste 21 SMA-Kinder, von denen vier zuvor eine VEPTR®Behandlung (durchschnittlich 2,5 Jahre) erhalten hatten und somit einen Stabwechsel erhielten; 17 Kinder waren nicht spinal voroperiert. Allen Kindern wurde das oben genannte extern auszufahrende MAGEC®-System implantiert, welches primär nach fünf Monaten und anschließend alle drei Monate verlängert wurde. Klinische, radiologische (Skoliosekrümmungswinkel, Beckenschiefstand, Kyphose, Lordose, Wirbelsäulenlänge) und Komplikationsdaten wurden erfasst und statistisch ausgewertet.

In der Gruppe der Primärimplantierten ( $\mathrm{n}=17)$ konnte der Skoliosekrümmungswinkel der Hauptkrümmung um $57,5 \%$ von $70^{\circ}\left(26^{\circ}-98^{\circ}\right)$ auf $30^{\circ}\left(3-62^{\circ}\right)$ gesenkt und diese Korrektur im Verlauf gehalten werden $\left(31^{\circ}\right.$ nach 2,4 Jahren follow-up). Die voroperierte Gruppe $(\mathrm{n}=4)$ wies durch die MAGEC®-Implantation eine Verminderung des Skoliosekrümmungswinkels um $41,2 \%$ auf $\left(31^{\circ} \mathrm{zu} 18^{\circ}\right)$. Durch die bilaterale Verankerung des Stabsystems von Rippen zu Beckenkamm konnte der Beckenschiefstand in der nicht voroperierten Gruppe ( $\mathrm{n}=17)$ initial um $75 \%$ gesenkt werden $\left(17^{\circ} \mathrm{zu} 4^{\circ}\right)$ und blieb auch während der Beobachtungszeit stabil. Die Kinder der voroperierten Gruppe $(\mathrm{n}=4)$ wiesen eine Initialreduktion des Beckenschiefstandes von 37,5\% auf. Sowohl die Kyphose als auch die Lordose konnten weder initial noch im 
Verlauf auf Normwerte korrigiert werden. Thorakal zeigte sich eine Tendenz zur Hyperkyphose, lumbal konnte eine Abflachung des sagittalen Profils beobachtet werden. Durch die Implantation nahm die Wirbelsäulenlänge initial um mehr als $50 \mathrm{~mm}$ zu, während des ersten Beobachtungsjahrs wurde ein Zuwachs von $12 \mathrm{~mm}$ verzeichnet. Es ergab sich eine Komplikationsrate von 6,5\% (21 von 325 Interventionen). Dabei wurden in drei Fällen Revisionsoperationen durchgeführt. Die häufigste Komplikation war eine Nichtdistrahierbarkeit des Implantates (13 von 304 Distraktionen, 4,3\%).

Es konnte gezeigt werden, dass transkutan distrahierbare magnetische Systeme ein geeignetes Mittel sind, um bei Kindern mit SMA und Wirbelsäulendeformität den Skoliosekrümmungswinkel zu reduzieren und im Untersuchungsverlauf zu stabilisieren, den Beckenschiefstand zu korrigieren und eine Wirbelsäulenlängenzunahme zu gewährleisten bei vergleichsweise geringer Komplikationsrate. Durch die externe transkutane Distraktion wird eine operative Verlängerung für etwa zwei bis drei Jahre obsolet. Hiermit kann vermutlich besonders für SMA-Kinder mit eingeschränkter pulmonaler Situation eine Verbesserung der Lebensqualität erreicht werden. Da das beschriebene operative Verfahren die Wirbelsäule selbst nicht tangiert, ist die intrathekale Medikamentenapplikation, welche im Rahmen der neu zugelassenen Therapie mit Nusinersen notwendig werden kann, nicht eingeschränkt. 


\section{$6 \quad$ Literaturverzeichnis}

Akbarnia BA, Emans JB (2010): Complications of growth-sparing surgery in early onset scoliosis. Spine $\underline{35}, 2193-2204$

Akbarnia BA, Marks DSF, Boachie-Adjei O, Thompson AGF, Asher MAM (2005): Dual Growing Rod Technique for the Treatment of Progressive Early-Onset Scoliosis: A Multicenter Study. J Neurosurg Spine $\underline{30}$, S46-S57

Akbarnia BA, Campbell RM, Dimeglio A, Flynn JM, Redding GJ, Sponseller PD, Vitale MG, Yazici M (2011): Fusionless procedures for the management of early-onset spine deformities in 2011: what do we know? J Child Orthop $\underline{5}$, 159-172

Akbarnia BA, Cheung K, Noordeen HF (Orth), Elsebaie HM, Yazici MM| |, Dannawi ZF (Orth), Kabirian N (2013): Next Generation of Growth-Sparing Techniques: Preliminary Clinical Results of a Magnetically Controlled Growing Rod in 14 Patients With EarlyOnset Scoliosis. Spine 38, 665-670

Arakawa R, Arakawa M, Kaneko K, Otsuki N, Aoki R, Saito K (2016): Imaging Flow Cytometry Analysis to Identify Differences of Survival Motor Neuron Protein Expression in Patients With Spinal Muscular Atrophy. Pediatr Neurol 61, 70-75

Bowen RE, Scaduto AA, Banuelos S (2008): Decreased body mass index and restrictive lung disease in congenital thoracic scoliosis. J Pediatr Orthop 28 , 665-668

Brunner R, Gebhard F (2002): Neurogene Wirbelsäulendeformitäten. Orthopäde $\underline{31}$, 51-57

Burnett BG, Crawford TO, Sumner CJ (2009): Emerging treatment options for spinal muscular atrophy. Curr Treat Options Neurol 11, 90-101

Byers RK, Banker BQ (1961): Infantile muscular atrophy. Arch Neurol $\underline{5}, 140-164$

Campbell RM Jr, Smith MD (2007): Thoracic Insufficiency Syndrome and Exotic Scoliosis. J Bone Joint Surg $\underline{89}-\underline{A}, 108-122$

Campbell RM Jr, Smith MD, Mayes TC, Mangos JA, Willey-Courand DB, Kose N, Pinero RF, Alder ME, Duong HL, Surber JL (2003): The Characteristics of Thoracic Insufficiency Syndrome Associated with Fused Ribs and Congenital Scoliosis. J Bone Joint Surg Am 85- $\underline{\mathrm{A}}, 399-408$

Campbell RM Jr, Smith MD, Hell-Vocke AK (2004a): Expansion thoracoplasty: the surgical technique of opening-wedge thoracostomy. Surgical technique. J Bone Joint Surg Am $\underline{86}-\underline{A}, 51-64$

Campbell RM Jr, Smith MD, Mayes TC, Mangos JA, Willey-Courand DB, Kose N, Pinero RF, Alder ME, Duong HL, Surber JL (2004b): The Effect of Opening Wedge Thoracostomy 
on Thoracic Insufficiency Syndrome Associated with Fused Ribs and Congenital Scoliosis. J Bone Joint Surg Am $\underline{86}-\underline{A}, 1659-1674$

Canavese F, Dimeglio A (2013): Normal and abnormal spine and thoracic cage development. World J Orthop $\underline{4}, 167-174$

Canavese F, Rousset M, Le Gledic B, Samba A, Dimeglio A (2014): Surgical advances in the treatment of neuromuscular scoliosis. World J Orthop $\underline{5}, 124-133$

Carstens C (1999): Die neuromuskuläre Skoliose. Orthopäde 28, 622-633

Castro D, Iannaccone ST (2014): Spinal muscular atrophy: therapeutic strategies. Curr Treat Options Neurol $\underline{16}, 1-11$

Chandran S, McCarthy J, Noonan K, Mann D, Nemeth B, Guiliani T (2011): Early treatment of scoliosis with growing rods in children with severe spinal muscular atrophy: a preliminary report. J Pediatr Orthop $\underline{31}, 450-454$

Cheung KM, Cheung JP, Samartzis D, Mak KC, Wong YW, Cheung WY, Akbarnia BA, Luk KD (2012): Magnetically controlled growing rods for severe spinal curvature in young children: a prospective case series. Lancet $\underline{379}, 1967-1974$

Cheung JPY, Yiu KKL, Samartzis D, Kwan K, Tan BB, Cheung KM (2018): Rod lengthening with the magnetically controlled growing rod: factors influencing rod slippage and reduced gains during distractions. Spine $\underline{43}$, E399-E405

Choi E, Yaszay B, Mundis G, Hosseini P, Pawelek J, Alanay A, Berk H, Cheung K, Demirkiran G, Ferguson J, et al. (2017): Implant Complications After Magnetically Controlled Growing Rods for Early Onset Scoliosis: A Multicenter Retrospective Review. J Pediatr Orthop $\underline{37}$, e588-e592

Chua K, Tan CY, Chen Z, Wong HK, Lee EH, Tay SKH, Ong HT, Goh DYT, Hui JHP (2016): Long-term Follow-up of Pulmonary Function and Scoliosis in Patients With Duchenne's Muscular Dystrophy and Spinal Muscular Atrophy. J Pediatr Orthop $\underline{36}, 63$ 69

Cobb J (1948): Outline for the study of scoliosis. Am Acad Orthop Surg 5, 261-275

Crijns TJ, Stadhouder A, Smit TH (2017): Restrained Differential Growth: The Initiating Event of Adolescent Idiopathic Scoliosis. Spine 42, E726-E732

Emans JB, Caubet JF, Ordonez CL, Lee EY, Ciarlo M (2005): The treatment of spine and chest wall deformities with fused ribs by expansion thoracostomy and insertion of vertical expandable prosthetic titanium rib: growth of thoracic spine and improvement of lung volumes. Spine $\underline{30}, 58-68$

Evans GA, Drennan JC, Russman BS (1981): Functional classification and orthopaedic management of spinal muscular atrophy. J Bone Joint Surg Br $\underline{63 \mathrm{~B}}, 516-522$ 
Farley FA, Li Y, Jong NB, Powell CC, Speers MSB, Childers DMM, Caird MS (2014):

Congenital Scoliosis SRS-22 Outcomes in Children Treated With Observation, Surgery, and VEPTR. Spine $\underline{39}, 1868-1874$

Finkel RS, Chiriboga CA, Vajsar J, Day JW, Montes J, De Vivo DC, Yamashita M, Rigo F, Hung G, Schneider E, et al. (2016): Treatment of infantile-onset spinal muscular atrophy with nusinersen: a phase 2, open-label, dose-escalation study. Lancet $\underline{388}$, 3017-3026

Fletcher ND, Bruce RW (2012): Early onset scoliosis: current concepts and controversies.

Curr Rev Musculoskelet Med $\underline{5}, 102-110$

Forst R, Ingenhorst A, Mortier W: Neuromuskuläre Systemerkrankungen. In: Zichner L

(Hrsg.): Systemerkrankungen. 1. Auflage; Thieme, Stuttgart 2003, 223-253

Fujak A, Raab W, Schuh A, Kreß A, Forst R, Forst J (2012): Operative treatment of scoliosis in proximal spinal muscular atrophy: results of 41 patients. Arch Orthop Trauma Surg $\underline{132}, 1697-1706$

Fujak A, Raab W, Schuh A, Richter S, Forst R, Forst J (2013): Natural course of scoliosis in proximal spinal muscular atrophy type II and IIIa: descriptive clinical study with retrospective data collection of 126 patients. BMC Musculoskelet Disord 14, 1-5

Gantner AS, Braunschweig L, Tsaknakis K, Lorenz HM, Hell AK (2018): Spinal deformity changes in children with long-term vertical expandable prosthetic titanium rib (VEPTR) treatment. Spine J $\underline{18}, 567-574$

Giglio CA, Volpon JB (2007): Development and evaluation of thoracic kyphosis and lumbar lordosis during growth. J Child Orthop 1, 187-193

Gillingham BL, Fan RA, Akbarnia BA (2006): Early onset idiopathic scoliosis. J Am Acad Orthop Surg 14, 101-112

Gomez JA, Lee JK, Kim PD, Roye DP, Vitale MG (2011): „Growth friendly“ spine surgery: management options for the young child with scoliosis. J Am Acad Orthop Surg $\underline{19}, 722$ 727

Granata C, Merlini L, Magni E, Marini ML, Stagni SB (1989): Spinal Muscular Atrophy: Natural History and Orthopaedic Treatment of Scoliosis. Spine 14, 760-762

Groenefeld BM, Hell AK (2013): Ossifications after Vertical Expandable Prosthetic Titanium Rib Treatment in Children With Thoracic Insufficiency Syndrome and Scoliosis. Spine $\underline{38}$, E819-E823

Grönefeld B: Das Problem der Ossifikation und Implantat- Wanderung nach VEPTR (vertical expandable prosthetic titanium rib)-Implantation bei Kindern und Jugendlichen mit Thorax-Insuffizienz-Syndrom und Skoliose. Med. Diss. Göttingen 2012 
Grönefeld K: Skoliose-Therapie bei Kindern und Jugendlichen mit dem MAGEC® (magnetic expansion control)-Spinalsystem in Kombination mit dem VEPTR (vertical expandable prosthetic titanium rib)-Instrumentarium. Med. Diss. Göttingen (in Vorbereitung, mit freundlicher Genehmigung der Verfasserin)

Haaker G, Fujak A (2013): Proximal spinal muscular atrophy: current orthopedic perspective. Appl Clin Genet $\underline{6}, 113-120$

Hasler C-C, Mehrkens A, Hefti F (2010): Efficacy and safety of VEPTR instrumentation for progressive spine deformities in young children without rib fusions. Eur Spine J $\underline{19}, 400$ 408

Hedequist D, Emans J (2004): Congenital scoliosis. J Am Acad Orthop Surg 12, 266-275

Hell AK, Campbell RM, Hefti F (2005): Neues Therapiekonzept für Kinder mit

Thoraxinsuffizienz-Syndrom aufgrund von Wirbelsäulenfehlbildungen. Klin Padiatr 217, 268-273

Hell AK, Groenefeld K, Tsaknakis K, Braunschweig L, Lorenz H (2018): Combining bilateral magnetically controlled implants inserted parallel to the spine with rib to pelvis fixation surgical technique and early results. Clin Spine Surg $\underline{31}$, 239-246

Herold G: Innere Medizin 2014. Gerd Herold (Verlag), Köln 2013

Hickey BA, Towriss C, Baxter G, Yasso S, James S, Jones A, Howes J, Davies P, Ahuja S (2014): Early experience of MAGEC magnetic growing rods in the treatment of early onset scoliosis. Eur Spine J 23 Suppl 1, S61-65

Hosseini P, Pawelek JB, Mundis G, Yaszay B, Ferguson J, Helenius I, Cheung K, Demirkiran G, Alanay A, Senkoylu A, et al. (2016): Magnetically-Controlled Growing Rods for Early Onset Scoliosis: A Multicenter Study of 23 Cases with Minimum 2 Years Follow-Up. Spine $\underline{41}, 1456-1462$

Kromeyer-Hauschild K, Wabitsch M, Kunze D, Geller F, Geiß HC, Hesse V, Hippel A von, Jaeger U, Johnsen D, Korte W, et al. (2001): Perzentile für den Body-mass-Index für das Kindes- und Jugendalter unter Heranziehung verschiedener deutscher Stichproben. Monatsschr Kinderheilkd 149, 807-818

Kwan KYH, Cheung JPY, Yiu KKL, Cheung KMC (2018): Ten year follow-up of JarchoLevin syndrome with thoracic insufficiency treated by VEPTR and MCGR VEPTR hybrid. Eur Spine J 27, 287-291

La Rosa G, Oggiano L, Ruzzini L (2017): Magnetically Controlled Growing Rods for the Management of Early-onset Scoliosis: A Preliminary Report. J Pediatr Orthop 37, 79-85 
Lattig F, Taurman R, Hell AK (2012): Treatment of Early-Onset Spinal Deformity (EOSD) With VEPTR: A Challenge for the Final Correction Spondylodesis-A Case Series. J Spinal Disorder Tech 29, E246-E251

Lau D, Clark AJ, Scheer JK, Daubs MD, Coe JD, Paonessa KJ, LaGrone MO, Kasten MD, Amaral RA, Trobisch PD, et al. (2014): Proximal junctional kyphosis and failure after spinal deformity surgery: a systematic review of the literature as a background to classification development. Spine $\underline{39}$, 2093-2102

Lebon J, Batailler C, Wargny M, Choufani E, Violas P, Fron D, Kieffer J, Accadbled F, Cunin V, De Gauzy JS (2017): Magnetically controlled growing rod in early onset scoliosis: a 30case multicenter study. Eur Spine J 26, 1567-1576

Lee J, Park Y-S (2016): Proximal Junctional Kyphosis: Diagnosis, Pathogenesis, and Treatment. Asian Spine J 10, 593-600

Lefebvre S, Bürglen L, Reboullet S, Clermont O, Burlet P, Viollet L, Benichou B, Cruaud C, Millasseau P, Zeviani M (1995): Identification and characterization of a spinal muscular atrophy-determining gene. Cell $\underline{80}, 155-165$

Lefebvre S, Burlet P, Liu Q, Bertrandy S, Clermont O, Munnich A, Dreyfuss G, Melki J (1997): Correlation between severity and SMN protein level in spinal muscular atrophy. Nat Genet 16, 265-269

Lorenz H, Badwan B, Hecker M, Tsaknakis K, Groenefeld K, Braunschweig L, Hell AK (2017): Magnetically controlled devices parallel to the spine in children with spinal muscular atrophy. JBJS Open Access $\underline{2}$, e0036

Ludolph AC: Motoneuronenerkrankungen. In: Berlit P (Hrsg.): Klinische Neurologie. 2. Auflage; Springer, Heidelberg 2005, 541-560

Ludolph AC: Amyotrophe Lateralsklerose und andere Motoneuronenerkrankungen. In: Hacke W (Hrsg.): Neurologie. 14. Auflage; Springer, Berlin Heidelberg 2015, 813-826

Lunn MR, Wang CH (2008): Spinal muscular atrophy. Lancet 371, 2120-2133

Maharshi V, Hasan S (2017): Nusinersen: The First Option Beyond Supportive Care for Spinal Muscular Atrophy. Clin Drug Investig 37, 807-817

Markowitz JA, Singh P, Darras BT (2012): Spinal muscular atrophy: a clinical and research update. Pediatr Neurol $\underline{46}, 1-12$

Master DL, Son-Hing JPM, Poe-Kochert CR, Armstrong DGM, Thompson GH (2011): Risk Factors for Major Complications after Surgery for Neuromuscular Scoliosis. Spine $\underline{36}$, $564-571$ 
Mayer OH, Redding G (2009): Early changes in pulmonary function after vertical expandable prosthetic titanium rib insertion in children with thoracic insufficiency syndrome. J Pediatr Orthop 29, 35-38

McElroy MJ, Shaner AC, Crawford TO, Thompson GH, Kadakia RV, Akbarnia BA, Skaggs DL, Emans JB, Sponseller PD (2011): Growing rods for scoliosis in spinal muscular atrophy: structural effects, complications, and hospital stays. Spine $\underline{36}, 1305-1311$

Mercuri E, Bertini E, Iannaccone ST (2012): Childhood spinal muscular atrophy: controversies and challenges. Lancet Neurol $\underline{11}, 443-452$

Morrissy RT, Goldsmith GS, Hall EC, Kehl D, Cowie GH (1990): Measurement of the Cobb angle on radiographs of patients who have scoliosis. Evaluation of intrinsic error. J Bone Joint Surg Am 르르, 320-327

Noordeen HM, Shah SA, Elsebaie HB, Garrido EE, Farooq N, Al Mukhtar M (2011): In Vivo Distraction Force and Length Measurements of Growing Rods: Which Factors Influence the Ability to Lengthen? Spine $\underline{36}$, 2299-2303

Putzier M, Groß C, Zahn RK, Pumberger M, Strube P (2016): Besonderheiten neuromuskulärer Skoliosen. Orthopäde $\underline{45}, 500-508$

Ridderbusch K, Rupprecht M, Kunkel P, Hagemann C, Stucker R (2017): Preliminary Results of Magnetically Controlled Growing Rods for Early Onset Scoliosis. J Pediatr Orthop $\underline{37}$, e575-e580

Roberts DF, Chavez J, Court SD (1970): The genetic component in child mortality. Arch Dis Child $\underline{45}, 33-38$

Rumalla K, Yarbrough CK, Pugely AJ, Koester L, Dorward IG (2016): Spinal fusion for pediatric neuromuscular scoliosis: national trends, complications, and in-hospital outcomes. J Neurosurg Spine 25, 500-508

Rushton PRP, Siddique I, Crawford R, Birch N, Gibson MJ, Hutton MJ (2017): Magnetically controlled growing rods in the treatment of early-onset scoliosis: A Note Of Caution. J Bone Joint Surg $\underline{\text { 99-B}}$, 708-713

Russman BS (2007): Spinal muscular atrophy: clinical classification and disease heterogeneity. J Child Neurol 22, 946-951

Russman BS, Iannacone ST, Buncher CR, Samaha FJ, White M, Perkins B, Zimmerman L, Smith C, Burhans K, Barker L (1992): Spinal muscular atrophy: new thoughts on the pathogenesis and classification schema. J Child Neurol ㄱ, 347-353

Samdani AF, Ranade A, Dolch HJ, Williams R, St Hilaire T, Cahill P, Betz RR (2009): Bilateral use of the vertical expandable prosthetic titanium rib attached to the pelvis: a novel treatment for scoliosis in the growing spine. J Neurosurg Spine 10, 287-292 
Sanders JO (2007): Maturity indicators in spinal deformity. J Bone Joint Surg Am $\underline{89}-\underline{A}, 14-20$ Sankar WN, Skaggs DL, Yazici M, Johnston CE, Shah SA, Javidan P, Kadakia RV, Day TF, Akbarnia BA (2011): Lengthening of dual growing rods and the law of diminishing returns. Spine $\underline{36}, 806-809$

Skaggs DL, Akbarnia BA, Flynn JM, Myung KS, Sponseller PD, Vitale MG, Chest Wall and Spine Deformity Study Group, Growing Spine Study Group, Pediatric Orthopaedic Society of North America, Scoliosis Research Society Growing Spine Study Committee (2014): A classification of growth friendly spine implants. J Pediatr Orthop $\underline{34}$, 260-274 Sporer SM, Smith BG (2003): Hip dislocation in patients with spinal muscular atrophy. J Pediatr Orthop 23, 10-14

Sucato DJM (2007): Spine Deformity in Spinal Muscular Atrophy. J Bone Joint Surg $\underline{89}$ - $\underline{\mathrm{A}}$, $148-154$

Teoh KH, von Ruhland C, Evans SL, James SH, Jones A, Howes J, Davies PR, Ahuja S (2016): Metallosis following implantation of magnetically controlled growing rods in the treatment of scoliosis: A case series. J Bone Joint Surg $\underline{98}-\underline{B}, 1662-1667$

Thompson W, Thakar C, Rolton DJ, Wilson-MacDonald J, Nnadi C (2016): The use of magnetically-controlled growing rods to treat children with early-onset scoliosis: early radiological results in 19 children. Bone Joint J $\underline{98}-\underline{B}, 1240-1247$

Tis JE, Karlin LI, Akbarnia BA, Blakemore LC, Thompson GH, McCarthy RE, Tello CA, Mendelow MJ, Southern EP, Growing Spine Committee of the Scoliosis Research Society (2012): Early onset scoliosis: modern treatment and results. J Pediatr Orthop $\underline{32}$, 647-657

Trobisch P, Suess O, Schwab F (2010): Die idiopathische Skoliose. Dtsch Arztebl Int 2010 $\underline{107}, 875-884$

Ulfig N: Kurzlehrbuch Neuroanatomie. 1. Auflage; Thieme, Stuttgart New York 2008

Vai S, Bianchi ML, Moroni I, Mastella C, Broggi F, Morandi L, Arnoldi MT, Bussolino C, Baranello G (2015): Bone and Spinal Muscular Atrophy. Bone 9ㅗ, 116-120

Vitale MG, Matsumoto H, Roye DP, Gomez JA, Betz RR, Emans JB, Skaggs DL, Smith JT, Song KM, Campbell RM (2008): Health-related quality of life in children with thoracic insufficiency syndrome. J Pediatr Orthop 28, 239-243

Vry J, Schubert IJ, Semler O, Haug V, Schönau E, Kirschner J (2014): Whole-body vibration training in children with Duchenne muscular dystrophy and spinal muscular atrophy. Eur J Paediatr Neurol 18, 140-149

Watts SL (2016): Use of a Vertical Expandable Prosthetic Titanium Rib in Children With Thoracic Insufficiency Syndrome and Scoliosis. Crit Care Nurse 36, 52-61 
Ziemann U: Motoneuronale Erkrankungen. In: Steinmetz H, Sitzer M (Hrsg.): Lehrbuch Neurologie. 1. Auflage; Urban \& Fischer/Elsevier, München 2011, 309-321

Zivkovic V, Büchler P, Ovadia D, Riise R, Stuecker R, Hasler C (2014): Extraspinal ossifications after implantation of vertical expandable prosthetic titanium ribs (VEPTRs). J Child Orthop $\underline{8}, 237-244$

\section{Internetquellen:}

Bedienungsanleitung MAGEC® (2014). http://ellipse-tech.com/wpcontent/uploads/2015/06/LC0002-P-GERMAN-Artwork.pdf; Zugriff am 21.08.2017

Biogen Presseinformation zur Zulassung von Spinraza ${ }^{\circledR}$ (2017). http://www.initiative-sma.de; Zugriff am 19.08.2017

Biogen Community-Update zur Zulassung von Spinraza ${ }^{\circledR}(2017)$.

http://www.muskelstiftung.de/Dokumente/biogen_sma-community_spinraza_euzulassung_update_01-06-2017.pdf; Zugriff am 26.06.2017

BMI nach Perzentilen im Kindes- und Jugendalter. http://aga.adipositasgesellschaft.de/mybmi4kids/; Zugriff am 12.11.2016

Centricity. http://www3.gehealthcare.de/de-de/footer_links/impressum; Zugriff am 12.11.2016

Cheung W-Y, Luk KD-K (2013): Classification of adolescent idiopathic scoliosis. http://www.boneandjoint.org.uk/sites/default/files/Classification $\% 20$ of $\% 20$ Adolescent \%20Idiopathic\%20Scoliosis.pdf; Zugriff am 26.06.2016

CoughAssist E70. https://hul.de/wpcontent/uploads/2014/10/HUL_BRO_Cough_6_Seiter_210x297_low.pdf; Zugriff am 12.11.2016

DePuy Synthes. http://emea.depuysynthes.com/locations/dede?pmid $=\mathrm{c0a} 80237 \mathrm{~cd} 31038$ befcacb201 fd7b069\&page $=0 \&$ searchData=veptr; Zugriff am 18.09.2017

ixserv. http://www.ixmid.com/index.php/kontakt; Zugriff am 12.11.2016

Kinder BMI Rechner der Uni Tübingen. http://www.medizin.unituebingen.de/kinderklinik/de/abteilungen/allgemeine-paediatrie-haematologieonkologie/sprechstunden/endokrinologie-adipositas/bmi-rechner/; Zugriff am 12.11.2016

Kirschner J, Müller-Felber W, Schara U (2016): Härtefallprogramm zur Behandlung der Spinalen Muskelatrophie Stellungnahme der deutschen Behandlungszentren. 
http://www.muskelstiftung.de/Dokumente/EAP_Stellungnahme_Deutsche-

Zentren_17-10-2016.pdf; Zugriff am 12.11.2016

MAGEC® Patientenbroschüre. https://www.nuvasive.com/wp-

content/uploads/2017/03/MAGEC-Patient-Education-Brochure-US.pdf; Zugriff am 30.07.2017

MAGEC® Zulassung in Europa. http://ellipse-tech.com/PR/CE-Mark-ApprovalMAGEC.pdf; Zugriff am 31.07.2017

Microsoft. http://www.microsoft.com/de-de/corporate/rechtliche-

hinweise/impressum.aspx; Zugriff am 12.11.2016

STATISTICA. http://www.statsoft.de; Zugriff am 12.11.2016 


\section{Danksagung}

Mein Dank gilt meiner Doktormutter Frau Prof. Dr. med. Anna-K. Hell, die mir die Gelegenheit zur Promotion ermöglichte. Insbesondere bedanke ich mich für die Unterstützung, die ich während meines Praktischen Jahres erfuhr, wodurch die Vollendung dieser Arbeit erst möglich wurde.

Dem Betreuer meiner Doktorarbeit, Dr. med. Heiko Lorenz, gilt mein ganz herzlicher Dank. Ich erlebte eine Hingabe ohnegleichen und einen Zuspruch und Optimismus, wenn ich diesen ganz besonders benötigte.

Dr. Lena Braunschweig, die mir bei der Erstellung der Graphen und der Statistik zur Seite stand, möchte ich an dieser Stelle ebenfalls aufrichtig danken. 